\title{
Report on the TESLA Engineering Study/Review
}

\author{
July 8,2002
}

C. Boffo ${ }^{1}$, H. Carter ${ }^{1}$, Y. Cho ${ }^{2}$, W. Chronis ${ }^{3}$, J. Cornuelle ${ }^{4}$, P. Czarapata ${ }^{1}$, J. Delayen $^{3}$, T. Dombeck ${ }^{1}$, H. Edwards ${ }^{*}, 1$, D. Finley ${ }^{1}$, M. Foley ${ }^{1}$, P. H. Garbincius ${ }^{*}, 1$, C. Jensen ${ }^{1}$,

A. Klebaner ${ }^{1}$,V. Kuchler ${ }^{1}$, T. Lavine ${ }^{4}$, T. Nicol ${ }^{1}$, J. Noonan ${ }^{2}$, H. Padamsee ${ }^{5}$, T. Page ${ }^{1}$, R. Pasquinelli ${ }^{1}$, T. Peterson ${ }^{1}$, H. Pfeffer ${ }^{1}$, G. Pile ${ }^{2}$, J. Preble ${ }^{3}$, R. Rabehl ${ }^{1}$, M. Ross ${ }^{4}$,

A. Rowe ${ }^{1}$, R. Stefanski ${ }^{1}$, J. Theilacker ${ }^{1}$, J.G. Weisend $\mathrm{II}^{4}$, and D. Wolff ${ }^{1}$.

${ }^{1}$ Fermi National Accelerator Laboratory, Batavia, IL;

2 Argonne National Laboratory, Argonne, IL;

${ }^{3}$ Thomas Jefferson National Accelerator Facility, Newport News, VA;

4 SLAC, Stanford Linear Accelerator Center, Stanford, CA;

${ }^{5}$ Cornell University, Ithaca, NY; and

*study leaders, editors, and contacts: hedwards@fnal.gov, garbincius@fnal.gov

Fermilab TM-2179 and available electronically at home.fnal.gov/ peterg/report 


\section{Table of Contents}

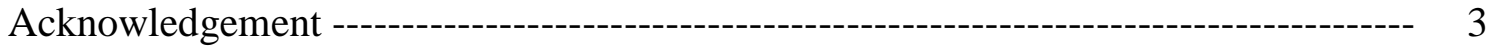

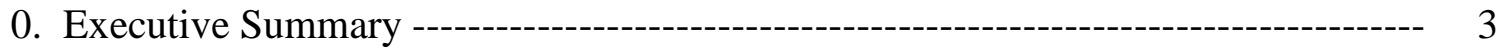

I. Introduction ---

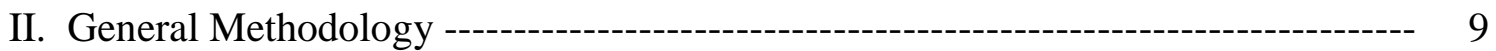

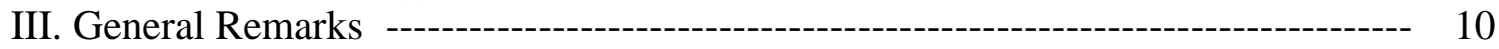

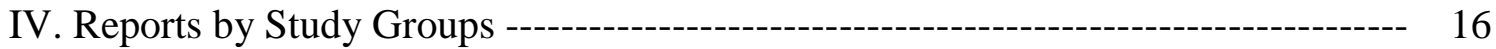

A. Main Linac Modules

1. RF Cavities ----or 17

2. RF Input Couplers ---------o- 32

3. Cryomodules ----------o--- 36

4. Concluding Remarks ---_- 48

B. Main Linac RF System

1. High Power RF - Klystrons and Modulators ----------------- 54

2. RF Distribution, Low Level RF, and Operating Margin ----------- 57

3. Industrial Studies, Costs, and Commentary ------------------ 60

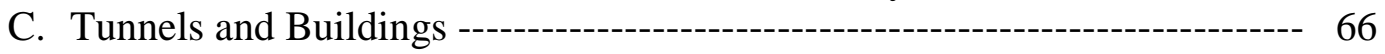

D. Machine Infrastructure

1. Conventional Utilities and Systems --------------------------- 76

2. Cryogenics -----o- 77

E. XFEL Increments -------------on 85

F. Project Coordination, Overview, Integration, Logistics, \& Management --- 90

V. Summary, Conclusions, and Recommendations ------------------ 119

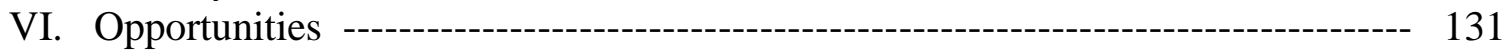

Appendices

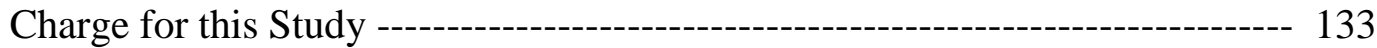

Participants, Working Groups, TESLA Contacts ------------------------ 135

References ---------------------------------------------------- 136

Glossary of U.S. and DOE Terms and Acronyms (common words) ---------- 137

Element Descriptions for Conventional Construction and Infrastructure ------ 139

Comparison of Costs for TTF, TESLA, CEBAF, and SNS Cryomodules ----- 146

TESLA Budget Book - Dictionary Version (WBS elements) ---------------- 149 


\section{Acknowledgement}

We wish to thank the many members of the TESLA collaboration for their patient, open and thoughtful discussions. This study would not have been possible without their full cooperation in supplying explanation and information. We hope we have recounted the information accurately. We are open to and encourage correction of errors we may have made.

We wish to acknowledge the help of the following individuals. We are sure the list is not complete. Our thanks also go to others who may, by oversight, be omitted here.

Danilo Barni, Wilhelm Bialowons, Reinhard Brinkmann, Stefan Choroba, Michael Ebert, Rolf Heuer, Markus Huening, Helmut Krech, Rolf Lange, Philippe Lebrun, Holger Lierl, Axel Matheisen, Wolf-Dietrich Möller, Carlo Pagani, Bernd Petersen, Dieter Proch, Hans Quack, Joerg Rossbach, Stefan Simrock, Dieter Trines, Hans Weise, Siegfried Wolff.

\section{Executive Summary}

A team from Argonne National Lab, Cornell, Fermilab, Jefferson Lab, and SLAC has studied the TESLA TDR and its associated cost and manpower estimates, concentrating on the five largest cost sub-systems (Main Linac Modules, Main Linac RF Systems, Civil Engineering, Machine Infrastructure, and XFEL Incremental). These elements were concerned mainly with providing energy reach. We did not study the lower cost, but still technically challenging elements providing luminosity and physics capability, namely damping rings, beam delivery system, beam injection system, positron production, polarized beams, etc. The study did not attempt to validate the TDR cost estimates, but rather its purpose was to understand the technology and status of the large cost items, and the methodology by which their estimated cost was determined. In addition, topics of project oversight were studied.

We find:

- TESLA R\&D activity is carried out by an international collaboration with US participation. The proposed TESLA project has been planned as an international project from its inception.

- A decade ago, a SCRF linear collider was a nice goal, but was considered by many to be prohibitive in terms of cost. Since then, the R\&D associated with the TESLA Test Facility (TTF) has made tremendous strides, attaining 250 MeV, a $0.1 \%$ scale proof of principle of each $250 \mathrm{GeV}$ linac, providing confidence, we believe, in the superconducting RF linac as a serious linear collider technology. 
- Although the purpose of this study was to understand the cost estimate methodology, and not to comprehensively validate the estimate, we find the methodology of the estimate to be reasonable in the form it was presented. The estimate was based on iterative TTF R\&D prototyping in industry and extrapolated to TESLA quantities through studies carried out with industry. These activities continue to refine and reduce the anticipated cost estimates. We find that the TTF experience, when extrapolated through mass-production, demonstrates the economic promise of this technology for a linear collider.

- TTF has operated with two cryomodules and injector (total of 17 cavities) for about 12,000 hours, mostly at $11 \mathrm{MV} / \mathrm{m}$ for the older module and $18.5 \mathrm{MV} / \mathrm{m}$ for the newer module, as determined by requirements of FEL studies and not by achievable gradient. Module 3 (AAC1) has operated for 1,000 hours at 20 $\mathrm{MV} / \mathrm{m}$ at mostly $5 \mathrm{~Hz}$. A beam of $\sim 250 \mathrm{MeV}$ has been supplied for FEL development and the FEL has demonstrated saturation in the $100 \mathrm{~nm}$ wavelength region.

- The TESLA TDR is based on a strategy of building the machine to initially operate at an energy of about $500 \mathrm{GeV}$, to explore the Higgs and related phenomena, and then to increase the energy to $800 \mathrm{GeV}$, to more fully explore the $\mathrm{TeV}$ energy scale. This strategy is in full accordance with the recommendations by ICFA, ECFA, and HEPAP. The TESLA TDR concentrated on the $500 \mathrm{GeV}$ capability. TTF must gain additional operational experience at this $23.4 \mathrm{MV} / \mathrm{m}$ gradient level. This is a major goal of the runs in 2002.

- The TDR described, furthermore, a strategy to reach $800 \mathrm{GeV}$. This strategy assumes that gradients of $35 \mathrm{MV} / \mathrm{m}$ can be obtained in mass production before the construction of the accelerator starts and that the RF couplers have the corresponding capabilities. Under these assumptions, TESLA will be able to raise the energy up to 700-750 GeV without hardware changes, however at a reduced $(\sim 1 / 5)$ luminosity. Operation with maximum luminosity at $800 \mathrm{GeV}$ would require the installation of additional RF and cryo-power. The cost for these upgraded power systems is not included in the estimate. Recently, a bare 9 cell $\mathrm{RF}$ cavity has been tested $\mathrm{CW}$ up to the required $35 \mathrm{MV} / \mathrm{m}$ gradient. Complete cryomodules and RF couplers have yet to be operated at this level with beam. Demonstration of this capability is the high priority of the TESLA Collaboration.

- The TESLA linear collider cost estimate at 3.136 B Euro and 6933 laboratory person-years is structured in a format suitable for a German project. It is not structured in a format as would be in a U.S. estimate for a similar project and does not include some of the elements that would be included in a U.S. estimate. Though we have made a comparison of these two approaches, we note that neither will be appropriate for an international project that will need to develop its own format. 
- In the TESLA estimate, manpower is expressed in person-years. This makes it easier to understand the resource requirements, and implicitly acknowledges the expected different labor basis from different collaborators. It also is the standard form for a German project. A U.S. estimate would include personnel cost explicitly. In a German project, escalation would be applied at project approval stage. Overhead functions and administrative resources would be covered in operating funds, and there is no contingency. A U.S. estimate for the Total Estimated Cost (TEC) would include the cost of personnel explicitly, administrative and general overheads (G\&A), commissioning activities, escalation, and especially contingency. We have tried to compare the total cost in German format (laboratory personnel, administrative personnel, and escalation included) using German labor rates with a U.S. format (personnel costs, G\&A, escalation and contingency) using U.S. labor rates. When the above (enumerated) elements are incorporated, the TESLA estimate in Euro becomes 4.34 B Euro, and a U.S. style estimate would be $\$ 6.1 \mathrm{~B}$, assuming a contingency assignment of $20 \%$. Labor rates play a large role in this difference. The incremental differential due to the labor rate model we chose, when G\&A, escalation and contingency are applied result in about $\$ 1.5 \mathrm{~B}$ of the $\$ 6.1 \mathrm{~B}$.

- The average cost to a company in Germany for industrial manpower (including benefits) is surprisingly only approximately $65 \%$ of comparable manpower costs in the Fermilab area. Average DESY laboratory personnel rates are $\sim 55 \%$. This reflects present international exchange rates ( $\$ 0.9 /$ Euro), not a cost of living normalization.

- The industrial mass production cost estimate model is based largely on single, pre-qualified companies providing components. However TESLA is trying to engage as many different suppliers of the large and critical components as possible in the R\&D so that there is a qualified competitive industrial pool. Both TESLA and our study team believe that single vendor contracts will not be appropriate for the actual project. Although a single vendor approach might minimize cost, it would limit the technology base and incur undue project risk. As the TESLA project would be international, critical systems will doubtless be shared between a number of collaborators, and the single vendor issue would be moot.

- The use of design and build contracting for tunnels, surface and underground buildings, conventional infrastructure, and even cryogenic plants has great merit, appears to be very cost effective, and should be considered for projects within the U.S. This might substantially reduce architect, engineering, and construction management (AECM) costs.

- The TESLA site plan is fixed. This plan provides for the ability to collide electrons with protons stored in HERA, fixes the geometry, and removes any consideration of alternate sites. The proposed layout allows for use of existing DESY infrastructure. 
- The allocation of the laboratory manpower to specific tasks was only presented in broad terms. It was difficult to understand how much manpower was allocated to on-site component testing, cavity tuning, RF processing, installation, and commissioning. Installation and commissioning plans (including beam) need development. There is no integrated resource-loaded schedule. This will be important to understand the material, manpower, and cost flow through the life of the project.

- There has been little analysis of project risks and their mitigation or contingency in terms of schedule or scope.

- There remain areas for continued review or further $R \& D$ and investigation. This would be true in any evolving project. Areas of possible review include: machine protection and failure mode analyses; operational considerations such as those associated with the positron production or alignment and vibration; continued analysis of accelerator physics aspects. The question of one or two tunnels would remain open for a Linear Collider based in the U.S.

- Examples are provided where the U.S. can immediately join in the R\&D and optimization activities for TESLA. Some of these include: kicker development, low level RF and RF distribution, modulator improvements, and advanced cavity R\&D. (Section IV.)

- We did not discuss in detail issues of internationalization or global collaboration on a Linear Collider project. TESLA has always proposed a truly global international project organization. It is clearly recognized by TESLA management that broad based discussions and proposals on global international project organization are timely and critical for further progress. These discussions should be independent of any LC technology choice. We believe there is a clear priority need to proceed with these organizational activities.

- The development of a management structure for TESLA project is just beginning. Although TTF is an international collaboration, it is managed as customary for high energy physics experiments. TESLA believes that developing a management model for a truly global project like a Linear Collider will be an important and major undertaking.

- We note the conundrum vis a vis on the one hand, the need to move ahead on project activities and decisions, and, on the other hand, getting collaborator participation in the decision processes at an early stage. By selecting many of the design choices and a site and tunnel configuration, TESLA has been able to concentrate on specific details in preparing the TDR proposal and cost estimate. However, these decisions will make it harder for future collaborators in the project to feel overall engagement. It is clearly understood by the TESLA collaboration that many aspects of the project will have to be reconsidered when 
the project collaboration is formed and new partners join. Even so we worry that TESLA may get too far in front of project collaborators in the technical decision process. Of course this problem becomes less were the level of collaborator activity to increase during the $R \& D$ phase. And the sooner a project organization can be formed, the better.

- The TESLA Collaboration has been able to make detailed progress on many advanced topics (R\&D, prototyping, TDR and cost estimate, environmental plan) using operating funds. These activities have been guided by collaboration meetings, workshops and regular internal reviews (and review at participating collaborator institutions) without excessive governmental monitoring.

- In the areas we reviewed, we believe that the TESLA proposal is sound and developed to an appropriate level of detail for this stage in the project proposal process. We congratulate the TESLA group on their efforts.

- As members of the international TESLA collaboration, several U.S. groups, both from universities and national laboratories, have been playing an active role in the development of the project. As outlined at several places in the report, there exist many opportunities for additional R\&D, which would be helpful and needed to further optimize various aspects of the design. This work should continue to be done within the framework of international collaboration.

- TESLA is clearly at a stage where the concepts of what makes an international laboratory, and what are its governance and political outreach structures need to be further developed. These are difficult problems that any LC will need to address along with the need for wide international participation. Developing these ideas needs to proceed in parallel with technical development. 


\section{Introduction}

In March, 2001, the TESLA Collaboration published its Technical Design Report (TDR, see references and links in Appendix), the first sentence of which stated "... TESLA (TeV-Energy Superconducting Linear Collider) (will be) a superconducting electron-positron collider of initially $500 \mathrm{GeV}$ total energy, extendable to $800 \mathrm{GeV}$, and an integrated X-ray laser laboratory." The TDR included cost and manpower estimates

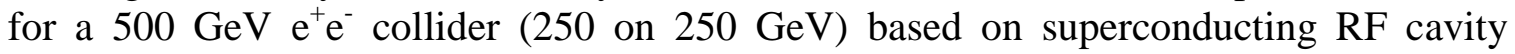
technology. This was submitted as a proposal to the German government. The government asked the German Science Council to evaluate this proposal. The recommendation from this body is anticipated to be available by November 2002. The government has indicated that it will react on this recommendation by mid-2003.

In June 2001, Steve Holmes, Fermilab's Associate Director for Accelerators, commissioned Helen Edwards and Peter Garbincius to organize a study of the TESLA Technical Design Report and the associated cost and manpower estimates. Since the elements and methodology used in producing the TESLA cost estimate were somewhat different from those used in preparing similar estimates for projects within the U.S., it is important to understand the similarities, differences, and equivalences between the TESLA estimate and U.S. cost estimates. In particular, the project cost estimate includes only purchased equipment, materials, and services, but not manpower from DESY or other TESLA collaborating institutions, which is listed separately. It does not include the R\&D on the TESLA Test Facility (TTF) nor the costs of preparing the TDR nor the costs of performing the conceptual studies so far. The manpower for the pre-operations commissioning program (up to beam) is included in the estimate, but not the electrical power or liquid Nitrogen (for initial cooldown of the cryogenics plant). There is no inclusion of any contingency or management reserve.

If the U.S. were to become involved with the TESLA project, either as a collaborator for an LC in Germany, or as host country for TESLA in the U.S., it is important to begin to understand the scope and technical details of the project, what R\&D still needs to be done, and how the U.S. can contribute. The charge for this study is included in the Appendix to this report. 


\section{General Methodology}

A group of approximately 30 people was assembled to perform this study. Some of these people were part of the U.S. TESLA Collaboration, having participated in R\&D leading to the Tesla Test Facility (TTF) and are authors of the TESLA TDR (March, 2001). The US institutions represented in this report include Argonne National Lab, Cornell University, Fermilab, Jefferson Lab, and SLAC. The five largest cost categories for the TESLA project were divided over six engineering study groups. A seventh group was assigned to study project management issues. Each of these seven groups were assigned one or two leaders who were responsible for coordinating the group's activities and for producing this report.

The information gathering activities included: studying the TESLA TDR and the cost elements represented in the TESLA Budget Book - dictionary version, briefings by Dieter Trines, the TESLA project leader, both at Fermilab and at Snowmass (July, 2001), e-mail correspondence between the study group leaders and their corresponding TESLA contacts, introductory presentations by all the group leaders to the entire study group (September-October, 2001), participation in the TTL/FEL TESLA Collaboration Meeting in Frascati (November, 2001), visits to DESY by many study group leaders including formal discussions with TESLA contact persons (November, 2001), and visits to Dresden and CERN to study the TESLA cryogenics system designs (November-December, 2001).

The study group leaders provided copies of their presentations and reports of their trips to Frascati, DESY, Dresden, and CERN, which were then edited into this report. The contents were first reviewed by all study group leaders, then sent to the TESLA collaboration management and participating U.S. lab management for their factual verification and for their comments and suggestions, especially regarding any sensitive items. After modification, the revised report was then submitted to Steve Holmes, the directors of the institutions participating in this study, and the director of DESY. 


\section{General Remarks on the Study}

The primary goal of this study was to become informed about the major components of the TESLA design. It focuses on the engineering aspects of the large cost items. (It does not look into the accelerator physics design of TESLA or of the smaller but complex systems like damping rings and beam delivery.) In this study, which was meant primarily as and educational activity, it is important to gain an understanding of the engineering design, its status, and evolution. From this basis, one can then understand the proposed project production schemes and cost models, which have been developed by TESLA.

The study groups heard descriptions of present TTF designs and cost. They then heard about Industrial Studies commissioned by DESY to begin to develop industrialization strategies and the cost estimate basis of the major systems.

The study group members were given access to detailed levels of the cost and manpower estimates. However, the TESLA management has requested that estimate numbers given in this report be limited for the most part to those already presented in the TDR. This request is to preserve the sensitive, confidential, and proprietary nature of some of the industrial estimates and negotiations.

In order to give a feeling for the lower level breakdown of some of the major system components, we will discuss costs presently associated with components produced for TTF and then give a discussion of the bottom line system costs relative to those estimated for the project.

\section{A. Project Model, Costs, Manpower, and Schedule}

The TESLA project cost estimate (TDR II, Chapter 10) is 3,136 M Euros for the Linear Collider and $241 \mathrm{M}$ Euros for the additional accelerator systems and civil engineering required for the XFEL. These costs are broken down by major subsystems in Figure 1. The laboratory manpower from DESY and other Collaborating TESLA Institutions is estimated to require 6933 person-years for the Linear Collider, Figure 2, and is not included in the cost estimate. For reference, the LEP and LHC projects both estimated approximately 5,000 man-years were needed from the CERN staff.

Roughly one-half of this TESLA laboratory manpower will be used for installation and commissioning. Also included is component testing and any tuning and processing of RF cavities on-site. However, these activities were not discussed in any detail.

Looking at these manpower needs in Figure 2, a very basic question arises: for example, will those numbers of skilled RF and Vacuum personnel be available at DESY and from the collaborating institutions? If not, then those tasks would have to be outsourced, thereby increasing the project costs. 


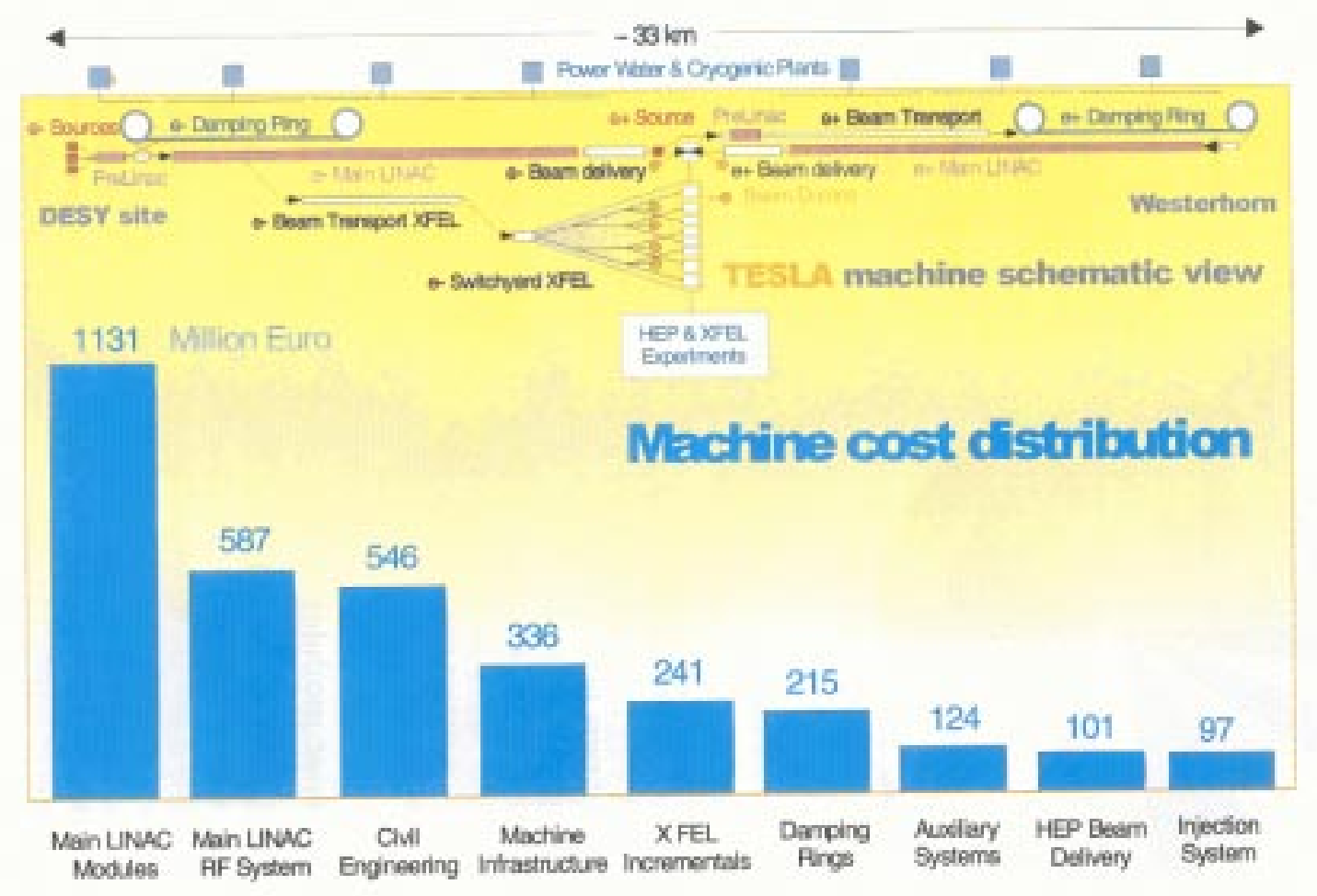

Figure 10.1.1: Onerwiew of the acolerator inestment coxts.

Figure 1. TESLA Cost Estimate Breakdown by Machine Subsystems. (TDR II, Ch. 10)

Figure 2. Laboratory manpower needs over 8 years of construction. (TDR II, Ch. 10)

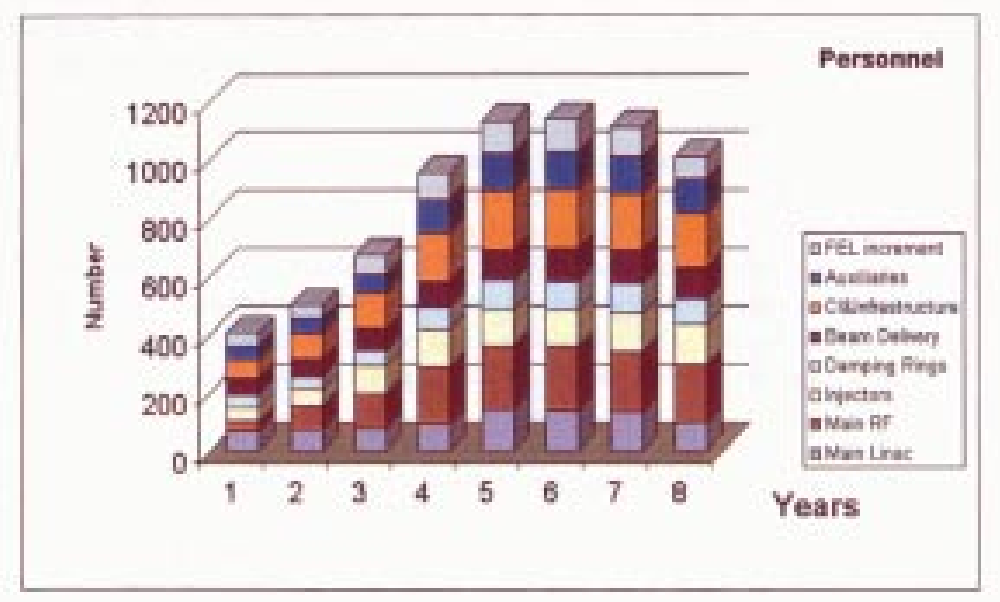

Figure 10.3.1: Labonatory manpouer noquirement for TESLA during the 8 years of wonstruction

Tesla Engineering Study Review 
The TESLA project schedule is based on an 8-year construction time scale. Prior to this, it is assumed that there is one year between project approval and construction start. During this time, bidding and award of major contracts will take place. For major machine components, 2 to 3.5 years will be needed from construction start to set up the necessary production facilities. Then, typically, the model plans for one year of ramp-up of production and 3 years of flat production rate. Installation of machine components is planned to begin after 4 years, and installation is to be completed after 8 years. The TESLA TDR schedules for civil construction and installation are shown in Figures 3 and 4.

The TDR design and associated cost estimate have been based, for the most part, on existing engineering technology as it has evolved at TTF. Many components developed for TTF have gone through a number of iterations of design and fabrication, resulting in technical improvements and cost savings. Potential cost savings of new and unproven technologies have not been used in the TESLA-500 estimate, e.g. electropolishing and superstructure. The assumption is that electropolishing, needed for $800 \mathrm{GeV}$ capable RF cavities, will be no more expensive than the present chemical buffered polishing process. Thus, it is expected that further iterations in design and development prior to construction may result in cost savings or technical contingency. We note that these design and prototype iterations are a form of value engineering. Ten years ago, a linac based on superconducting RF cavities was a nice goal, but the costs seemed prohibitive. Now, after the TTF R\&D, the costs are comparable for warm and cold RF linacs.

The production of major components has been analyzed using a one-contractor model. Our opinion of the wisdom of this approach will be commented on in our conclusions.

There was also discussion and confusion over the profit model assumed for the cavity and module production. Additional incentives considered were that TESLA could finance investment for infrastructure needed by contractors, and then the contractors would retain this infrastructure at the end of the contract. (There will be further later in this report and comments in the conclusion.) 


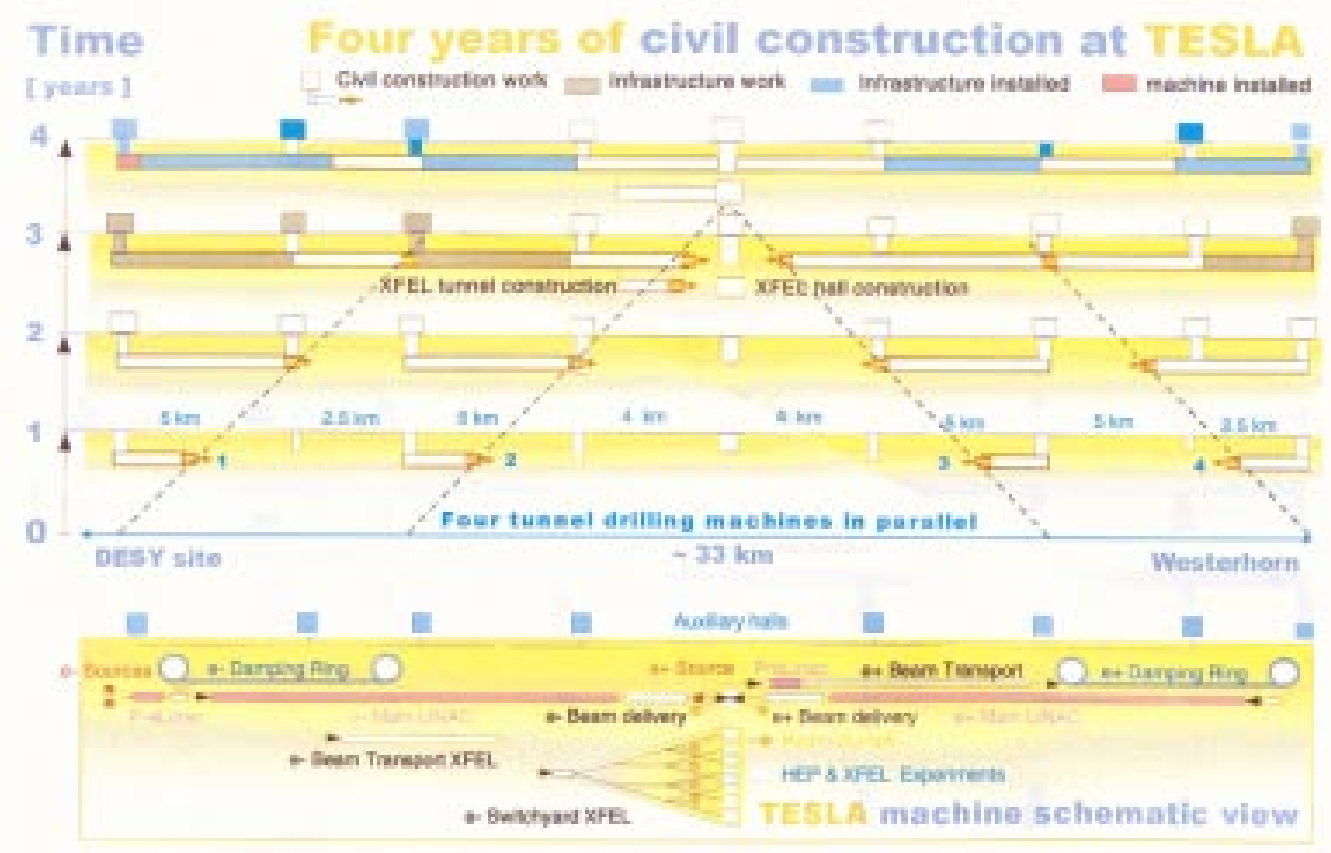

Pigure 10.5. 1: Time schedule for civil constriction.

Figure 3. Schedule for TESLA Civil Construction. (TESLA TDR II, Chapter 10)

Figure 4. Schedule for TESLA Machine Installation. (TESLA TDR II, Chapter 10)

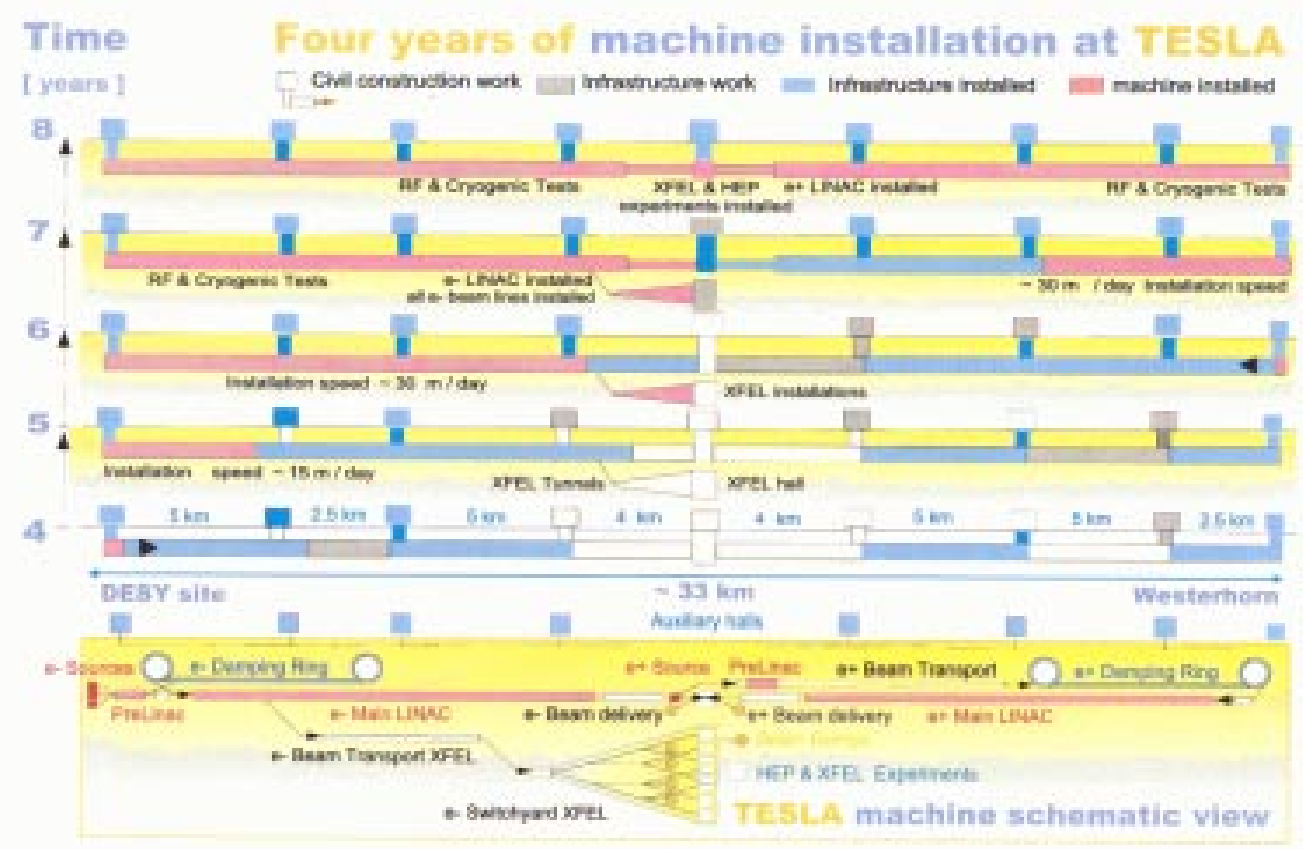

Fieure 10.5.2. Time solndule for mective isostellation. 


\section{B. Industrial Studies}

Much of the technical component part of the TESLA cost estimate has been based on industrial studies commissioned by DESY and/or the collaboration. These studies addressed possible production plans and strategies and provided a bottoms-up look at how production could be carried out and what its estimated cost would be. Major studies were typically of 6 months duration and $\sim \$ 100 \mathrm{~K}$ expense.

This cost estimating approach by industrial study is notable as it is not typical of procedures used in the U.S. at this phase of project planning.

The major industrial studies included:

Niobium production

Cavity fabrication

Cold mass

Cryomodule Assembly

Input RF Coupler

Klystron tubes

Modulators

Pulsed Modulator cables

Other RF components:

Directional Couplers:

Drivers:

Circulators \& loads: $400 \mathrm{~kW}$ circulators:
Wah Chang, Albany, OR, USA

Cabot Corp., Boyertown, PA, USA

Babcock Noell Nuclear GmbH, Wuerzburg, Germany, with Dornier consultant, and 2 additional studies by Accel and Zanon

E. Zanon SpA, Schio, Italy

two studies: Accel, Bergisch Gladbach, Germany and Noell-Babcock, with DESY and INFN (Zanon) advisors to both

Thomson-Thales, Velizy, France

Thomson-Thales

PPT Puls-Plasmatechnik GmbH, Dortmund, Germany - this study also included the pulse transformer (by $\mathrm{ABB}$ ), the IGCT switch (by ABB), and the HV power supplies for the modulators (by FUG Beerwald who build HVPS for new TTF modulators

Nexans Deutschland, Mönchengladbach, Germany

Spinner, Munich, Germany

SSB

Domen SPA Ferrite, St. Petersburg, Russia

AFT 
Other Cost Basis studies included:

Cryogenic Plants

Civil Construction estimate by Dr. H. Quack (Dresden) with consultants from

Linde AG Unternehmenszentrale, Araham Lincoln Strasse 21, 65189 Wiesbaden, Germany, and L'Air Liquide, Corporate Communications, 75 Quai d'Orsay, 75321 Paris cedex 07, France based on CERN studies \& LHC \& HERA experience

based on HERA construction experience in consultation with the firms of

Windels, Timm, \& Morgen, Ballindamm17, 90095 Hamburg, Germany, and

Amberg Ingenieurbüro AG, Trockenloostrsse 21, Postfach 27, CH-8105 Regensdorf-Watt, Switzerland

A discussion of cost scaling methods from a few items to mass-production quantities is included in Section IV.F. Project Coordination, Overview, Integration, Logistics, and Management. 


\section{Unit Quantities}

We list here some basic component quantities in an effort to reduce repetition in the following body of the report and to set an overall scale of the number of units required for the project.

$\begin{array}{ll}\text { TESLA-500 } & \text { Quantity } \\ \text { RF cavities } & 20592 \\ \text { Nb material } & 500 \text { tons } \\ \text { Input couplers } & 20592 \\ \text { Cryomodules } & 2574(12 \mathrm{~m}) \text { or } 1726(17 \mathrm{~m}) \\ \text { Quadrupole packages } & 720 \\ \text { Klystrons/Modulators } & 572 \\ \text { Cryo plants and halls } & 7 \\ \text { Cryo units } & 12(2.5 \mathrm{~km}, \sim 16 \text { strings of ten } 17 \mathrm{~m} \text { modules }) \\ \text { Vacuum barriers } & 4 \text { per cryounits }(\sim 500 \mathrm{~m} \text { separation }) \\ \text { Main Linac tunnel } & 33,500 \mathrm{~m}\end{array}$

\section{Issues Common throughout the Report}

Some issues will come up over and over in this report. Those recurring themes worth mentioning here include: contingency, the European cost model, Engineering, Design, Inspection, and Administration (EDIA), and DESY and collaborating institution personnel costs not included in the "project cost estimate".

\section{Reports by Study Groups}

In this section, we concentrate on the five largest cost elements in the TESLA Machine Cost Distribution (Figure 1, above) and Laboratory manpower requirements (Figure 2 and table above). To these, we add a study of TESLA project overview, coordination, installation, logistics, and management. We broke the Main Linac Modules into RF Cavities and Cryomodules, and the Main Linac RF System into Klystrons, Modulators and RF Distribution/Low Level RF. We also note that the TESLA Machine Infrastructure, has two main categories. AC Power, Water, and HVAC are combined with Tunnels and Buildings under our Civil Construction heading, and Cryogenics will be discussed under its own heading. 


\section{A. Main LINAC Modules}

\section{RF Cavities}

In addition to RF cavities, this section will briefly discuss power input couplers and cryomodule production, cavity/cryomodule integration, and testing plans. For reference, as an Appendix, there is a comparison of elements and costs for the equivalent cryomodule units for CEBAF, SNS, and TESLA TTF. An overview of module costs and reduction measure is given at the end of section III. A.

\section{a. Comments on Cavity Technical Status}

At the time of initiation of the TESLA cavity R\&D program about ten years ago, typical gradients achieved in production cavities at CDBAF were $\sim 8-10 \mathrm{MV} / \mathrm{m}$. Early on, the TESLA Collaboration chose for gradient goals $15 \mathrm{MV} / \mathrm{m}$ for a near term goal in the TESLA Test Facility (TTF), and $25 \mathrm{MV} / \mathrm{m}$ for the TESLA 500 long-term goal. More recently, with improvements in cavity packing factor, TESLA 500 can be achieved at a gradient of $23.4 \mathrm{MV} / \mathrm{m}$, and there is now a committed R\&D program to push gradients to the $35 \mathrm{MV} / \mathrm{m}$ level that would be needed for TESLA 800.

The TESLA collaboration has established an impressive pace for cavity production, preparation, testing, cryomodule population, and industrial production. Nearly 80 cavities have been fabricated by four industrial companies. These and other new cavities are being used to populate 10 cryomodules. Specifications and quality control procedures have been developed and qualified through the large number of cavities and cryomodules already produced.

Pushing for the highest gradients, some of the newest cryomodules (\# $1^{*}$ and \# 4) are populated by 16 cavities with gradients between 25 and $30 \mathrm{MV} / \mathrm{m}$ (in vertical dewar testing). Module \# 4 cavities showed consistent gradients up to $30 \mathrm{MV} / \mathrm{m}$ in a horizontal (Chechia) test with all the couplers and tuners attached and where the full klystron power was available (See Figure 1 for gradients.). During the horizontal test, all except one cavity showed gradients higher than $\mathrm{CW}$ results obtained in vertical cavity tests. This achievement shows that gradients above $30 \mathrm{MV} / \mathrm{m}$ can be reached when higher power is available as for the horizontal tests. The higher power also permits field emitters to be processed allowing higher gradients. (Vertical cavity acceptance tests are carried out without couplers and with only 200 watts of CW RF power.)

The spread in cavity performance is decreasing. Figure 2 shows a histogram of 9cell vertical dewar results for 56 cavities used to populate seven cryomodules. Note that 40 of these cavities achieved gradients between $25-32 \mathrm{MV} / \mathrm{m}$. The narrow spread in cavity performance is a good sign that QC (quality control) procedures and cavity specifications have matured considerably over the growth of TTF. 
Steady progress in gradients is obvious from three types of tests:

- bare cavity acceptance tests (called vertical tests);

- cavities fully equipped with couplers and tuners and tested with $200 \mathrm{~kW}$ or more of

RF power with the TESLA pulse length (called the horizontal test or CECHIA tests); high peak power conditioning (HPP) is possible at the MW level;

- and cavities tested in the tunnel inside complete cryomodules.

The spread in gradients among cavities within each module is also coming down (See the range bars of Figure 3).

When TTF was first put together, the average of vertical tests of cryomodule \# 1 was $18 \mathrm{MV} / \mathrm{m}$. For successive modules \# 2, 3, $1^{*}$, and 4, the cavity test average rose steadily to $22,25,27$, and $28 \mathrm{MV} / \mathrm{m}$. Following the same trend, the horizontal test result average rose from 16, 22, 24, 26, and $26 \mathrm{MV} / \mathrm{m}$.

Cryomodules \# 1, 2, and 3 installed in the tunnel demonstrated gradients of 13, 20 and $22 \mathrm{MV} / \mathrm{m}$. By proper phasing, one module will be run at greater than $20 \mathrm{MV} / \mathrm{m}$ for long times during TTF operation both for TESLA mode and FEL mode. To date, running the installed modules at high gradient has been limited, due to demands of the FEL SASE program. The ACC1 cryomodule is presently (March, 2002) operating at $21 \mathrm{MV} / \mathrm{m}$. Before the upcoming shut down of Summer 2002, the ambition is to install module \# 1 * and run it for a long period of time at the highest possible gradient. Cavities inside this module are capable of operating between $25-30 \mathrm{MV} / \mathrm{m}$.

After the shutdown of Summer 2002, the plan is to equip TTF2 with six cryomodules of high performance cavities. Three of these units $\left(1^{*}\right.$ and 4 and 5) are already populated.

Cryomodules will also be readied to evaluate the new technique of electropolishing (see below) and the concept of superstructures. Spare cryomodules filled with some of the weaker cavities will be readied for FEL operation less demanding of gradient. In all, plans call for ten cryomodules by Fall-Winter 2003-4 (6 installed in TTF2 including 1 electropolished, 2 spare, 1 "TESLA"-ep, 1 "FNAL"). Commissioning of TTF2 is planned for 3rd Quarter 2003. Prior to this time, the testing of 5 modules that have not already been tested is scheduled.

The BCP (buffered chemical polishing) etch of the niobium surface limits cavity performance to roughly $30 \mathrm{MV} / \mathrm{m}$. One way being explored to achieve $35 \mathrm{MV} / \mathrm{m}$ gradients needed for TESLA-800 is to electropolish the interior surface of the cavities instead of etching with BCP. Many single cell cavities have been electropolished by KEK in Japan and by a CERN/DESY collaboration. All exceed $30 \mathrm{MV} / \mathrm{m}$, and some reached $E_{a c c}=42 \mathrm{MV} / \mathrm{m}$. Electropolishing creates a much smoother surface.

Several 9-cell units have been electropolished at KEK and await testing at DESY. This testing has been delayed because of an accident in the high-pressure system that 
contaminated the water rinsing facility. It has taken several months to rebuild the system and re-qualify it. The plan is to outfit one module with electropolished cavities. As with any new procedure, it may take some time to achieve optimum results. TTF has also put together a new electropolishing facility, which they hope to commission shortly.

\section{b. Industrial Studies}

\section{i. General Remarks}

Now that individual structure gradients at the $25 \mathrm{MV} / \mathrm{m}$ level are well in hand, the TESLA collaboration has proceeded to industrial cost studies. Costs of major subsystems reported in the TESLA TDR were based on industrial studies conducted over six months. Several institutes were involved in cost evaluations. A planning group supervised layout of production and test facilities for mass production. The experience of many labs has been factored in. In particular the synergy between cryogenics and superconducting magnet expertise from the HERA project at DESY has been of great benefit to the TESLA project. Significant cost reductions have been realized by "value" engineering through several iterations of components. Overall, we saw several opportunities for US collaboration in the further development program for TESLA.

We note that the TESLA collaboration has the technology for cavity production, preparation, and cryomodule production so that companies cannot band together to raise prices during the project. From the TTF experience, TESLA will have the option to set up an independent factory.

We noted many strong points that bode well for TESLA's readiness to start construction of a linear collider by 2005 . Examples include:

- more than 40 of the one-meter long structures built by industries have been tested to gradients above $25 \mathrm{MV} / \mathrm{m}$;

- more than 5 cryomodules have been built and populated with 8 cavities each;

- cryomodule cost reduction has been achieved by incorporating 8 cavities in a module and by eliminating warm-cold transitions. Further reduction may be possible by increasing the number of cavities to 12 per cryomodule;

- gains have been achieved in cryogenics costs by integrating the cryogenic distribution system into the cryomodules;

- the first round of industrial studies have been completed for mass production and cost reduction; and

- future module cost reduction may be achieved through packaging cavities in the superstructure configuration of $2 \times 9$ cells with a single input coupler.

\section{ii. The Industrial Model}

Amongst the different possible models, the model developed for production planning and cost estimating assumed two major contractors: one to fabricate the cavities, a second to process the cavities and assemble the modules. We will call these Company 
A (cavity fabrication) and Company B ( processing and assembly). The cost studies for these production efforts used in the TDR estimate came from one company- BabcockNoell.

\section{Company A (cavity fabrication)}

Company A fabricates the bare cavities, which include helium vessel and vacuum end flanges, coupler ports, and HOM couplers. This company is responsible for accepting the $\mathrm{Nb}$ and $\mathrm{NbTi}$ material from the $\mathrm{Nb}$ supplier. Company A may, or may not, be responsible for managing the $\mathrm{Nb}$ supplier contract. It out-sources, or could carry out itself: deep-drawing of the cups, the machining of the cups (estimate based on outsourcing), tubes, flanges, HOM parts, etc. It is responsible for mechanical and RF measurements of the cups and dumbells. It prepares the parts for e-beam welding (ultra pure water and acid etch). It performs the e-beam welding and certifies its Q.C. It carries out final mechanical and vacuum inspection. Major equipment consists of four threechamber e-beam welders. Production rate must be 24 cavities/day. It is estimated that about 35 people will be needed.

\section{Company B (process and assembly)}

Company B receives components from many different suppliers and integrates them into a complete cryomodule. Components needed to "dress" the cavity include: the "bare" cavity, helium vessel, magnetic shielding, input coupler, and cold tuner. Components needed to assemble the module include the module "cold mass". This cold mass consists of the outer vacuum vessel, the gas return pipe (GRP), support posts, cryo piping, heat shields, super insulation, cavity, and quadrupole mounts. All components of the cold mass are supplied by its manufacturer. Other components needed for module assembly consist of the "dressed cavities", the quadrupole-steerer-BPM package, interconnects, instrumentation, etc.

Company $\mathrm{B}$ is responsible for cavity processing. Upon receipt of a cavity after incoming inspection, it must tune the cavity for field flatness, then etch (BCP) and oven bake it (1400C). After the oven bake, the cavity is etched to remove titanium deposited during the bake process. The cavity is then prepared for vertical dewar test. This consists of a light etch and high pressure rinsing (HPR). The cavity is then mounted (with clean room assembly) to a fixture that is made to hold 8 cavities with RF antennae. The insides of the cavities are to be evacuated for testing. The 8-cavity assembly is shipped to DESY where it will be tested in the Cryo Test Facility. DESY plans to test all cavities vertically.

After vertical tests, cavities are returned to Company B. It must then weld the cavity in the helium vessel, re-measure (warm bead pull), and re-clean it as necessary prior to clean room assembly of the inner input coupler part, and assembly of the string of cavities (8 or 12) and quad package. The string beam vacuum RF surface is sealed off prior to exit from the clean room. The cavity string is then mounted to the module gas return pipe (GRP), and aligned. Magnetic shields, thermal shields, piping, and superinsulation are installed. The assembly is then inserted into the vacuum vessel, 
connected via the support posts, and input coupler outer parts are connected. The module is then shipped to DESY where $25 \%$ will be tested in the Cryo Test Facility during peak production (100\% during production start up).

It is estimated that Company B's expense is about half in manpower, requiring 535 persons for 4 years. (The manpower estimate was modified somewhat from the industrial study to reflect more efficient assembly procedures than presently at TTF.) Cavity preparation is $\sim 50 \%$, and module assembly is $\sim 30 \%$.

\section{Other major companies}

$\mathrm{Nb}$ material-

Niobium material (plate) of 300 RRR quality is a major expense. There is one major supplier. The supplier will carry out inspection and eddy current scans of the disks. See discussion below.

Cryo vessel - cold mass-

The cold mass manufacturer supplies the cryo vessel with its associated gas return header, support posts, piping, thermal shields and mounts for the cavities. This company is key for making for ease of assembly, alignment, and tunnel installation.

Input couplers-

An RF input coupler is needed for each cavity. These couplers are about as costly to fabricate as the cavities themselves. They need RF processing by the fabricator.

\section{iii. Superconducting Material}

Approximately 500 tons of RRR (residual resistivity ratio) 300 niobium are needed to fabricate 21,000 TESLA-500 superconducting cavities. This is a small quantity of raw niobium as compared to the world production of niobium for steel and other products $(40,000$ tons per year). But it is a significant quantity for high RRR niobium, calling for multiple electron beam melting cycles and excellent vacuum in the beam melting furnace.

Detailed $\mathrm{Nb}$ specs have been developed over the last decade by joint efforts of Cornell, JLab and DESY. That $300 \mathrm{RRR}$ single cell Nb cavities reach $\mathrm{E}_{a c c}=42 \mathrm{MV} / \mathrm{m}$ shows that the $\mathrm{Nb}$ material specs needed for high gradients are understood and defined in the sense that they provide high field quality results. (The theoretical limit imposed by the thermodynamical critical field is about $50 \mathrm{MV} / \mathrm{m}$ for TESLA geometry.)

The TESLA Collaboration has developed a basic plan for material procurement. At present, no individual vendors can supply 500 tons of RRR 300 niobium over the fouryear cavity production schedule. For the TDR cost study, a collaboration was established 
with one of the leading $\mathrm{Nb}$ producers, Wah Chang (Oregon, USA). A plan was developed to upgrade their existing niobium production facilities to meet TESLA material quantity and composition specifications. Based on these discussions, Wah Chang has decided to proceed with improving one of their (smelting/purification) furnaces to be used solely for cavity niobium production, and to build another new furnace. The dedicated furnace increases the likelihood Wah Chang can achieve the niobium purity specifications as well as supply rate and quantity.

Wah Chang was not the only niobium supplier consulted for TESLA, however they were the most realistic option to incorporate into the cost estimates and production schedule. Besides Wah Chang, there are several industries that have supplied high RRR $\mathrm{Nb}$ for superconducting cavities: W.C. Heraeus, Tokyo Denkai, and Cabot. CBMM, Brazil is also interested in this market but remains to qualify. TESLA identified a Chinese firm that may be able to produce niobium with the required specifications and at a relatively low cost. Several companies could therefore be involved during the final procurement process.

The niobium supplier will perform all large-scale quality control (QC) on cavity material supplied to the cavity manufacturer. The niobium material will be supplied in two forms, stamped discs for half-cells and tubing for beam pipe end groups. Before arriving at the cavity manufacturer, all discs will be scanned for impurities and/or voids using proven eddy current mapping technologies. The niobium supplier will also spotcheck samples from every niobium batch for material composition and RRR. TESLA is confident that advances in QC during material production will ensure very low material failure and rejection rates. On a continuing basis, TTF is developing specific QC measures to further reduce material failure during cavity manufacture.

There are no material procurement problems that TESLA faces in the cavity flange and helium cryostat production. TESLA will need a significant supply of both titanium and niobium-titanium, however production will not impact current worldwide availability. Parts made of titanium and niobium-titanium are not critical to cavity performance and therefore no QC procedures beyond what the material suppliers perform are necessary.

High RRR Niobium production is an area for further exploration. A new industrial study is underway with Heraeus and Babcock-Noell to understand large scale production. This study combines the knowledge of a small company expert in niobium production with a large company knowledgeable in mass production. A German company experienced in metal powers production, Stark company, will carry out a $1000 \mathrm{~kg}$ study on how they would optimize RRR Nb production and cost. For the TDR, some cost reduction in material has been factored in, but a larger reduction may be possible. Thus, reduced material costs could provide a sizable contingency.

\section{iv. Cavity Production cost study}


The cavity production schedule calls for fabrication of 21,000 cavities total over four years. With a $1-2$ year ramp up in production rate, steady production of 24 cavities per day must be achieved and maintained for two years ending with a year to ramp down. The ability to produce cavities at a rate of 24 cavities per day drives the overall TESLA cryomodule installation schedule. See Figure 4 for relative cost elements for cavity production.

\section{v. Present cavity fabrication costs}

The first part of the DESY cavity cost study was to identify and evaluate the dominant cost drivers for cavity production. At present, prototypes for cavities are produced in several industrial companies: ACCEL, CERCA, Zanon, and Dornier (Dornier has since withdrawn from supplying this product). With existing facilities, TTF industry can weld a single cavity in 35 hours. Independent industrial studies were done by three companies with experience in TESLA 9-cell cavity production, and they came to very similar conclusions about the present cost distribution of the various manufacturing operations.

The total price at present for fabrication and niobium is about $50 \mathrm{~K}$ Euro per 9 cell cavity. This includes HOM coupler, input coupler port, transition from niobium beam pipe to liquid helium vessel, and niobium-titanium flanges. Fabrication cost to material cost is $2 / 3$ to $1 / 3$. The pie chart (Figure 4 ) shows the present fabrication cost distribution as determined by one of these studies. Electron beam welding turns out to be the driving component at 50\%. Machining is $36 \%$. The end groups cost as much as the cavity alone.

\section{vi. Cavity mass production facility}

An industrial study was carried out by Babcock-Noell to design a cavity production plant (Company A) to include special tooling and systems for mass production and cost reduction (Figure 5). Their study was based on the combination of the cavity experience of many laboratories and the industrial expertise of an experienced firm (Dornier). The study determined the costs of investment, start up, and close down. Infrastructure costs were included in the investment. It would take two years to start producing the first cavities. These large-scale production scenarios were solidly based on current industrial experience.

The planned cavity assembly production process is as follows. The fabrication facility either manufactures or receives all machined and formed components for the complete cavity from subcontractors. The components are cleaned, etched, and prepared for e-beam welding.

Through industrial modeling, Babcock-Noell determined that four large threechamber electron beam welding machines, each performing different assembly tasks, would provide adequate production capability. A three-chamber e-beam welding machine offers several advantages over single chamber e-beam welding machines. The primary advantage of multiple-chamber welding is the ability to perform several tasks 
simultaneously. The overhead associated with vacuum pump-down and component cooldown times is reduced to a minimum. In a three-chamber welder, the first chamber is used for jig and component set up, the second for welding, and the third for cool-down and component removal. A welding machine of this design keeps the middle chamber under constant vacuum, therefore keeping the welding volume extremely clean, a factor vitally important to weld integrity. The first and third chambers are pumped by separate massive vacuum systems capable of producing vacuum pressures matching the welding chamber in minutes. The set-up and cool-down times required in the first and third chambers are optimized with the welding time in the second chamber. With significant investment in welding and component assembly fixtures, many welds can be performed in a single throughput cycle with relatively little time wasted on vacuum pump-down.

Babcock-Noell provided an example of the capabilities of a three-chamber welding setup using a welding fixture design that allows $50 \mathrm{HOM}$ couplers to be welded in a single cycle.

At such a facility, they would be able to weld a cavity in 5 hours versus the present time required of 35 hours. The three-chamber welding machine and mass production techniques would bring down welding and cooldown time by a factor of 7 . Since the first study showed welding to be a major cost driver, this approach had a major overall impact on the cavity cost.

With four welding machines, the rate would increase to one cavity per hour. Production would take place 5 days per week, at 24 hours per day for 3 years. Two days per week would be scheduled for down time maintenance and upkeep. Start-up costs were taken into account in the estimate. Manpower costs were included to fabricate cavities. The company might take care of procuring the niobium (now done by DESY) and also the acceptance quality control on the material (now done by eddy current scanning at DESY). 5\% extra material would be purchased.

After implementation of welding and other cost cutting measures, the overall cost of bare cavity production was reduced by a large factor over the prototype costs. The largest component cost is outsourcing the parts, such as stamping cups from sheet, machining the cups, and making flanges. Further investigation may lead to reductions in this area. As far as possible, estimates were based on real quotes from subcontracting companies. The prime contractor would add several steps of quality control, such as measuring the RF frequency of the cups and dumbells.

In the two Babcock-Noell studies, industrial profit was not included. It is expected that this would be at most a 5\% increase. A savings of this magnitude could be realized if TESLA would provide pre-financing for infrastructure preparation. With this model in mind, no additional costs were added to these estimates. As an added benefit, ownership of the infrastructure would revert to the industrial companies. The new industry would retain its infrastructure after the last cavity is delivered to the cryomodule assembly facility and be free to seek other production contracts using the newly acquired capabilities. Subsequent business may come from a growing interest in fourth generation 
light sources around the world, (linac-based FELs and ERLs), accelerator transmutation of nuclear waste facilities, and intense proton linacs for future muon and neutrino sources. Electron beam welders and clean rooms are also generic facilities that are valuable for many large industrial processes. How reasonable a profit model this is or whether pre-financing by TESLA would save sufficiently to make up funds for profit remains to be seen. However, the use of project funds (if available) to finance industrial infrastructure development and start-up seems like an excellent way to expedite start-up and save overhead.

\section{vii. Cryomodule Integration}

Zanon conducted a cost study for the cryomodule cold mass with the help of other Italian firms. Both Babcock-Noell and Accel conducted industrial studies of cryomodule assembly (Company B). Details of the two plans vary, but overall estimates were consistent. Accel suggested 100\% testing of cavities; Babcock-Noell proposed $100 \%$ on startup, then $25 \%$ during full production. DESY decided to take on the $100 \%$ testing responsibility themselves. Testing will be carried out in The Cryo Test Facility planed at the DESY site. After the studies were carried out, a manpower effort adjustment was made, based on more recent experience in assembling TTF modules.

The Babcock-Noell study was finally used for the cost estimate. They took present methods of assembly and extended these to the large scale for TESLA. They added in schedule contingency by putting two shifts for production and one shift for maintenance. They did not analyze if there is a better more efficient approach as they did for cavity production. Upon review at DESY, it was found that they had used manpower numbers based on TTF experience with no efficiency reduction. Consequently, their manpower estimate was reduced somewhat. Another study will be commissioned for this step and is expected to bring further cost reduction.

The superconducting magnet package cost study was performed by a Spanish company, ACIA.

\section{viii. Testing plans, The Cryo Test Facility}

The plan is to carry out vertical dewar acceptance tests for all cavities. A set up is designed to test 8 cavities during a single cooldown cycle. Once consistent results demonstrate a reproducible and acceptable production process, a smaller sample of cavities could be tested at random. The cavity testing strategy still seems to be a somewhat open issue. Testing all cavities does open the possibility of sorting cavities to obtain modules of like performance cavities. Such a plan though operationally cumbersome, is still under consideration.

For cryomodules, DESY will test $100 \%$ of the modules during start-up production, then $25 \%$ during final module production. 
The test facility will be set up at the DESY site for both cavity tests and cryomodule tests. The facility is sized to accommodate the $25 \%$ module testing at maximum production rate. One HERA cold box (which supplies $4{ }^{\circ} \mathrm{K}$ and $80{ }^{\circ} \mathrm{K}$ ) supplemented by warm pumps in the test facility would provide the cryogenic capability of $50 \mathrm{gm} / \mathrm{sec}$ and provide $0.6 \mathrm{~kW} @ 2{ }^{\circ} \mathrm{K}, 31 \mathrm{mbar}$. Six module test benches and six large vertical dewar setups are planned. Module test rate is 1/2 module/day. (Tunnel installation rate is $30 \mathrm{~m} /$ day, $\sim$ two $17 \mathrm{~m}$ modules per day.) It is expected to a module test takes 9 days. (Coupler phase adjustment will be done on the tunnel.)

It will take three years to set up the testing facility followed by a training period of one year. Vertical dewar tests and module tests will proceed over four-five years phased with respect to initial training and to one another. The required building size is 83 m x $135 \mathrm{~m}$. (Building size for $100 \%$ module testing would not fit on the DESY site.) From the test facility, the cryomodules would be ready to go to the tunnel. A test plan has been defined. Manpower assigned for these tests is over 300 man-years peaking at about 70 individuals. Vertical tests employ $1 / 4$ to $1 / 3$ of the technician effort.

With a complete testing facility at TESLA, there would be full capability to repair and supply cryomodules during TESLA operation. The possibility is under discussion to add a cavity testing capability at the cavity string preparation facility to avoid shipping cavities or cavity strings back and forth. However, we note that the Test Facility investment is $~ 40 \mathrm{M}$ Euros, exclusive of the HERA plant (another 7-10 M Euros) and labor. It seems unlikely that testing at the module plant would be as reliable or cost effective.

\section{ix. Comparison of TESLA TDR cryomodule costs with other SCRF projects such as CEBAF and SNS}

The TESLA Main Linac cost estimates are equivalent to $53 \mathbf{K}$ Euros per active meter of RF structure. This should be compared to CEBAF costs of $\$ 206 \mathrm{~K}$ per active meter (1990 dollars) and to SNS (high- $\beta$ ) cryomodule costs of \$ 377 K (2001 dollars) per active meter. TESLA achieves a lower cost per meter (and much lower cost per MV, see Section IV A. 4) through improved cost effective design and larger numbers of modules. The main features are: increased effective filling factor, limited warm-cold transitions and integrated cryo distribution, longer cavities (more cells), and (higher gradient).

- TESLA is based on 9-cell cavity units, versus CEBAF which has 5-cell units and SNS which has 6-cell units;

- Each $17 \mathrm{~m}$ TESLA module carries 12 meters of active structure versus 4 meters and 3.6 meters for CEBAF and SNS, thereby increasing the cavity/cryomodule filling factor from $0.35-0.45$ to 0.7 ;

- TESLA modules incorporate the cryogenic transfer line, warm cold transitions take place only every $2.5 \mathrm{~km}$. This should be compared with other systems which use separate cryogenic transfer lines and have warm to cold transitions every module or so; 
- CEBAF cavities carry waveguide input and HOM couplers which are more bulky, made of niobium, and expensive;

- SNS cavities are $800 \mathrm{MHz}$ and larger in diameter, compared to CEBAF (1500 $\mathrm{MHz}$ ) and TESLA (1300 MHz); and finally,

- Large quantity production: CEBAF built 43 cyromodules, SNS is building 12 high- $\beta$ cryomodules, and TESLA will have 1716 cryomodules.

The appendix includes costing information for cavities and cryomodules for TTF R\&D, CEBAF, and SNS. 


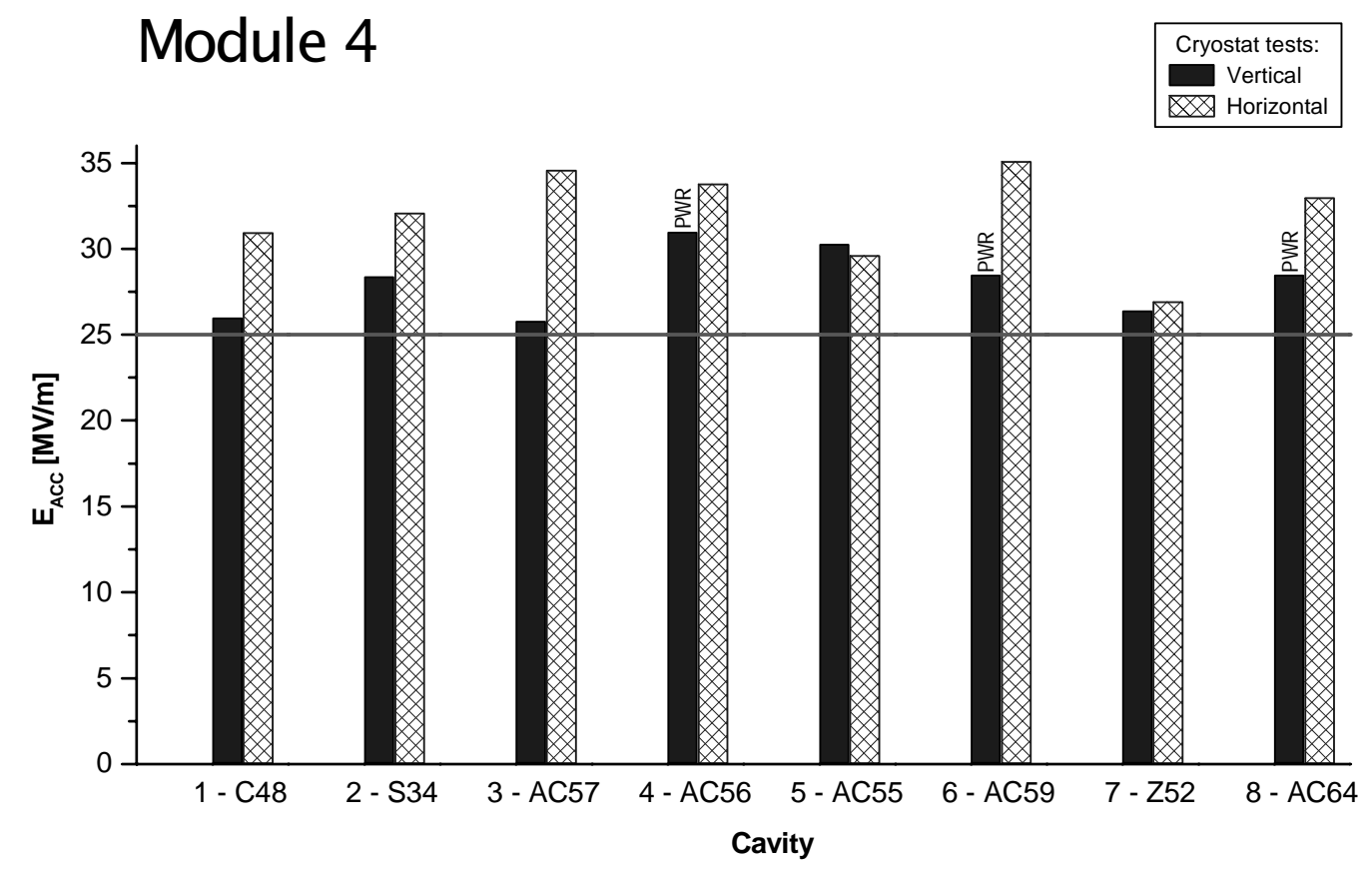

Figure 1. Accelerating gradients for RF cavities in Cryomodule \#4 for both horizontal and vertical tests.

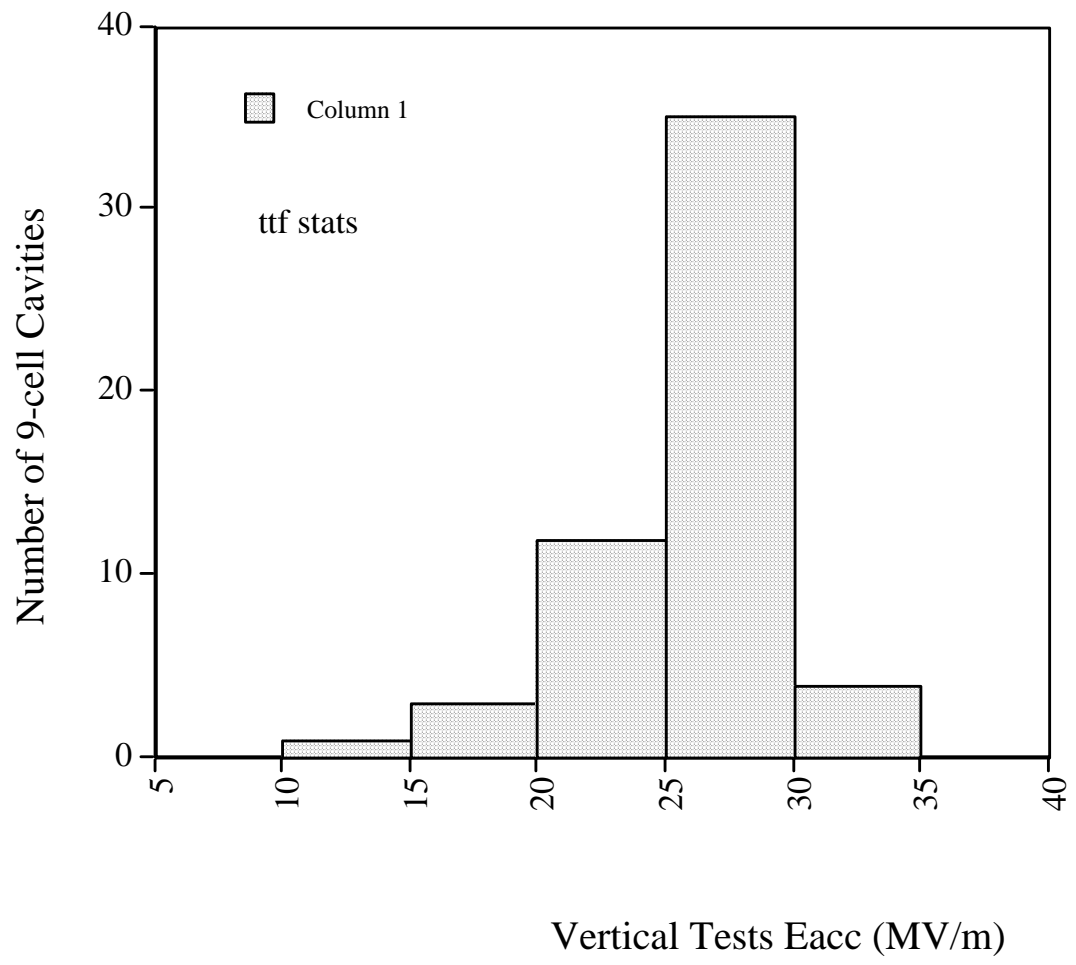

Figure 2. Distribution of accelerating gradients for 9-cell cavities for vertical testing. 

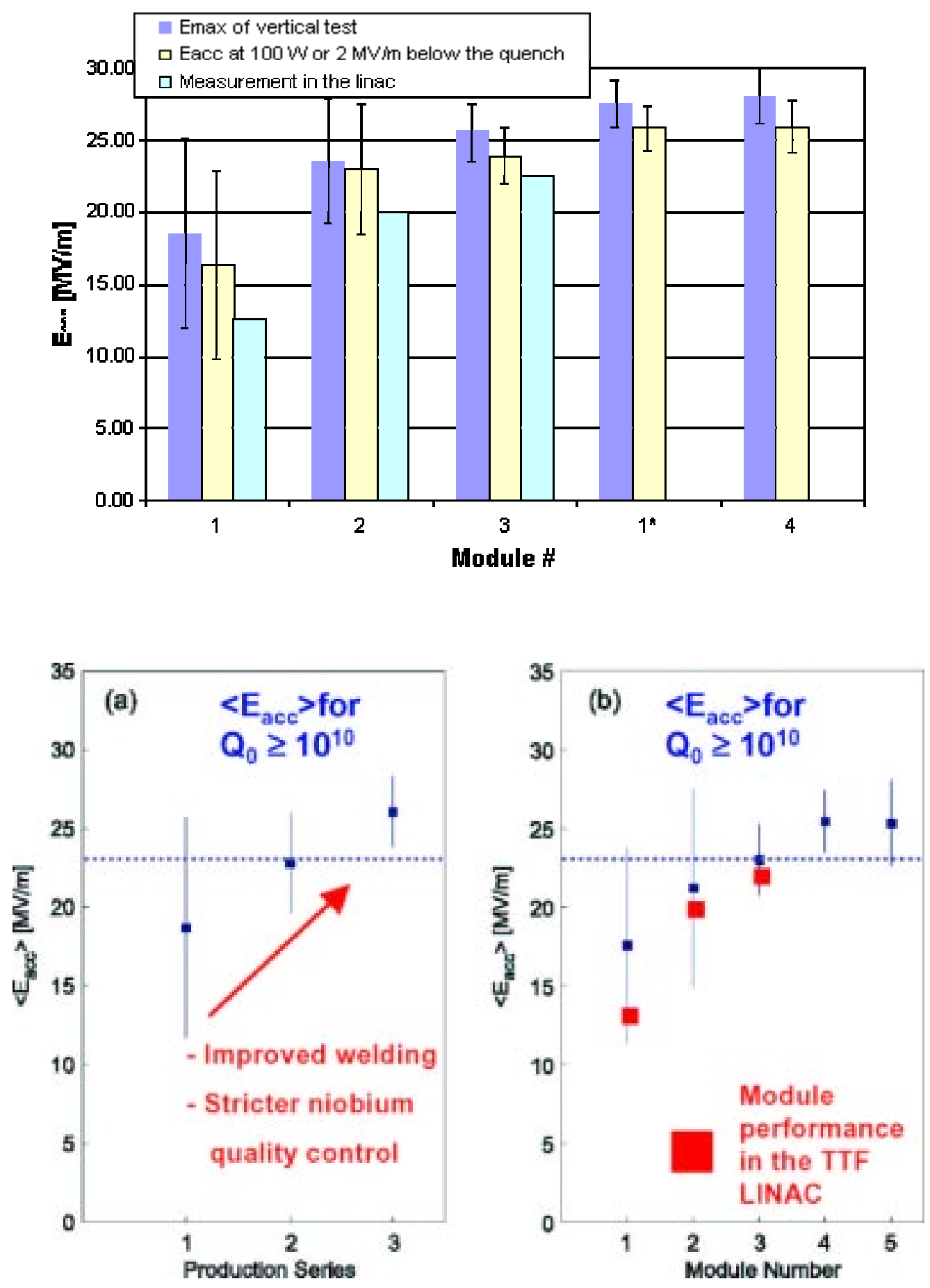

Figures $3 \mathrm{a}$ and $3 \mathrm{~b}$. Time dependence of average performance and spread in gradients.

The measurement in the linac is the average operating gradient.

Tesla Engineering Study Review 


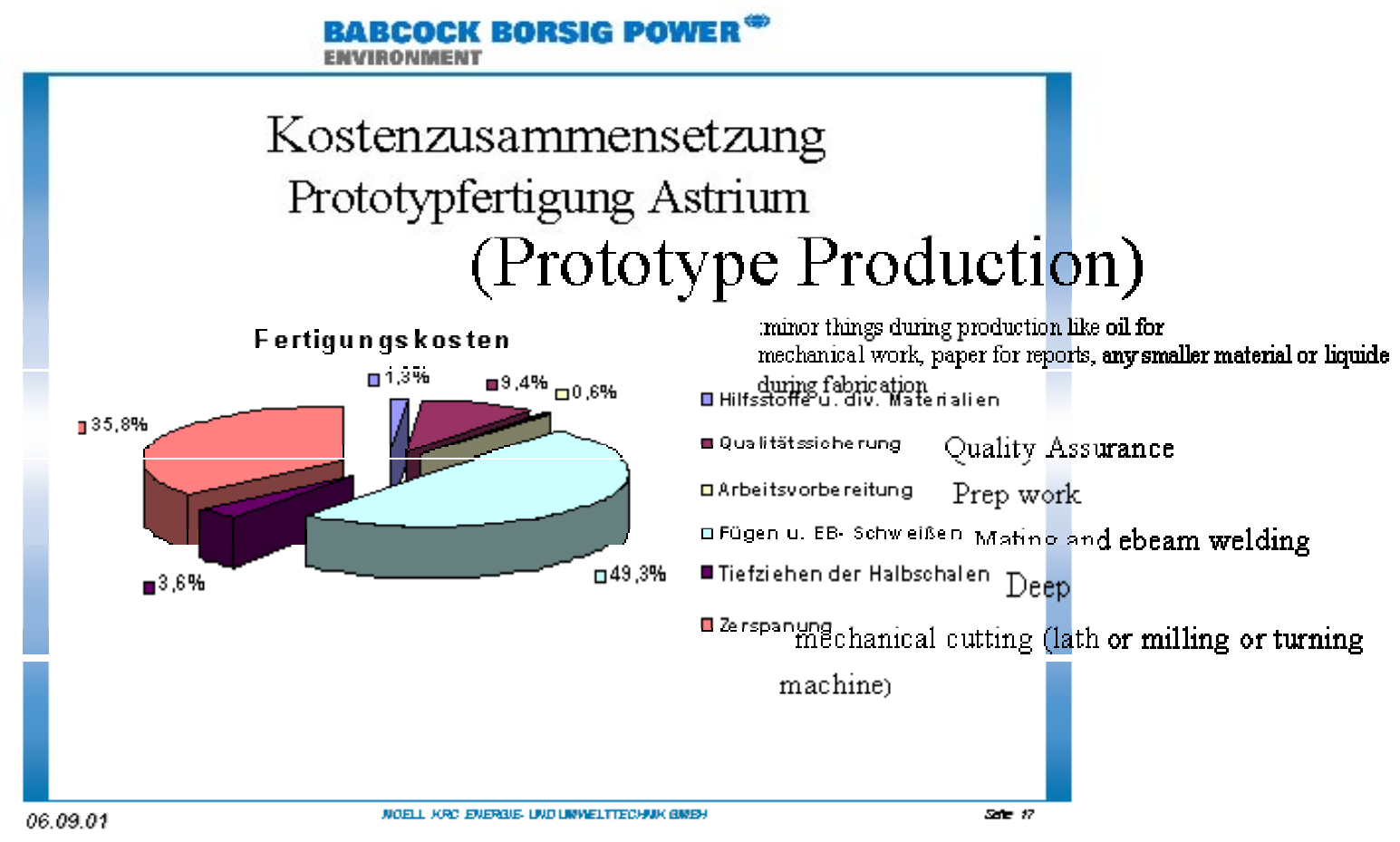

Figure 4. Relative cost elements for production of prototype RF cavities. 


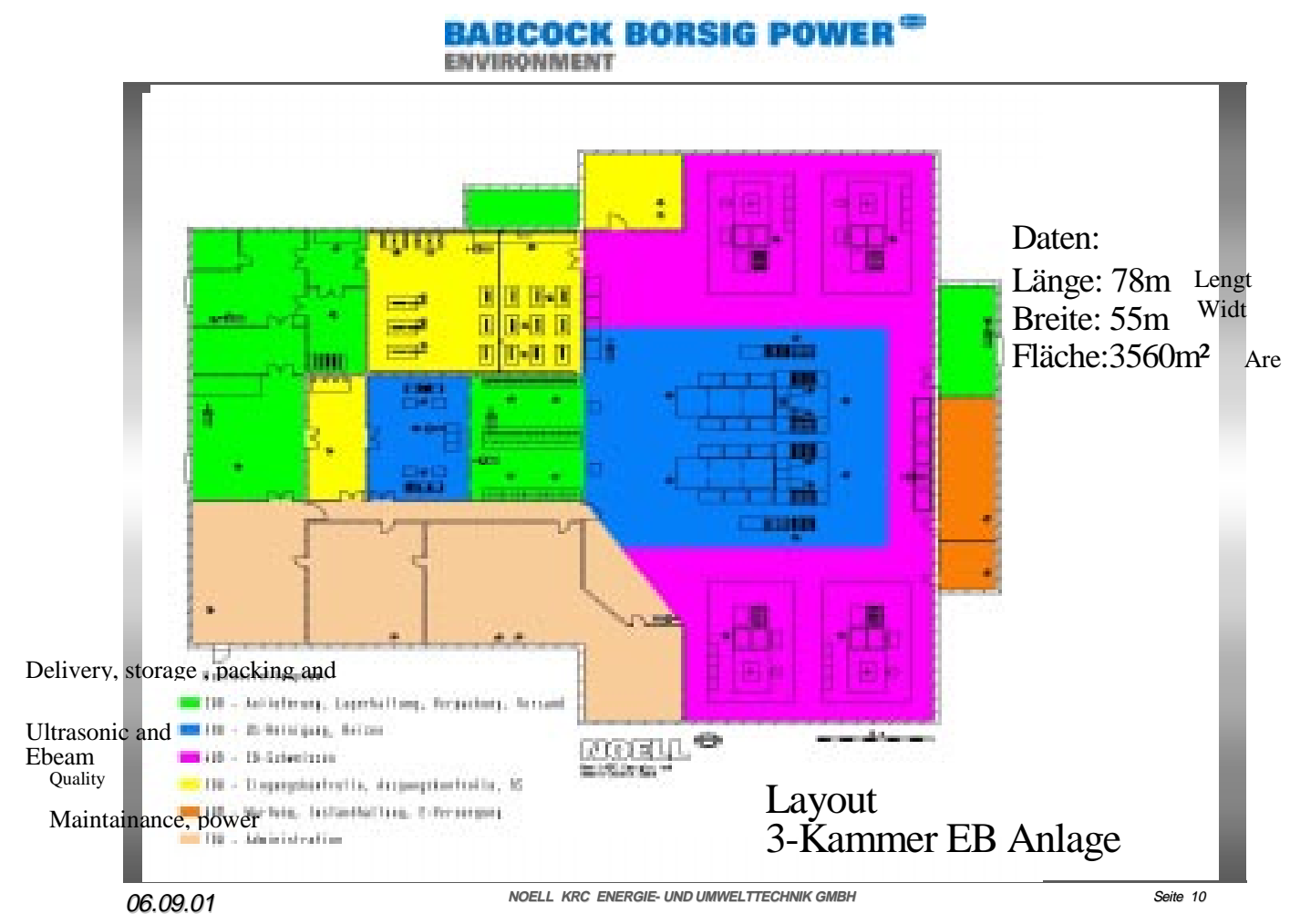

Figure 5. Conceptualization of Company A production plant for RF cavities

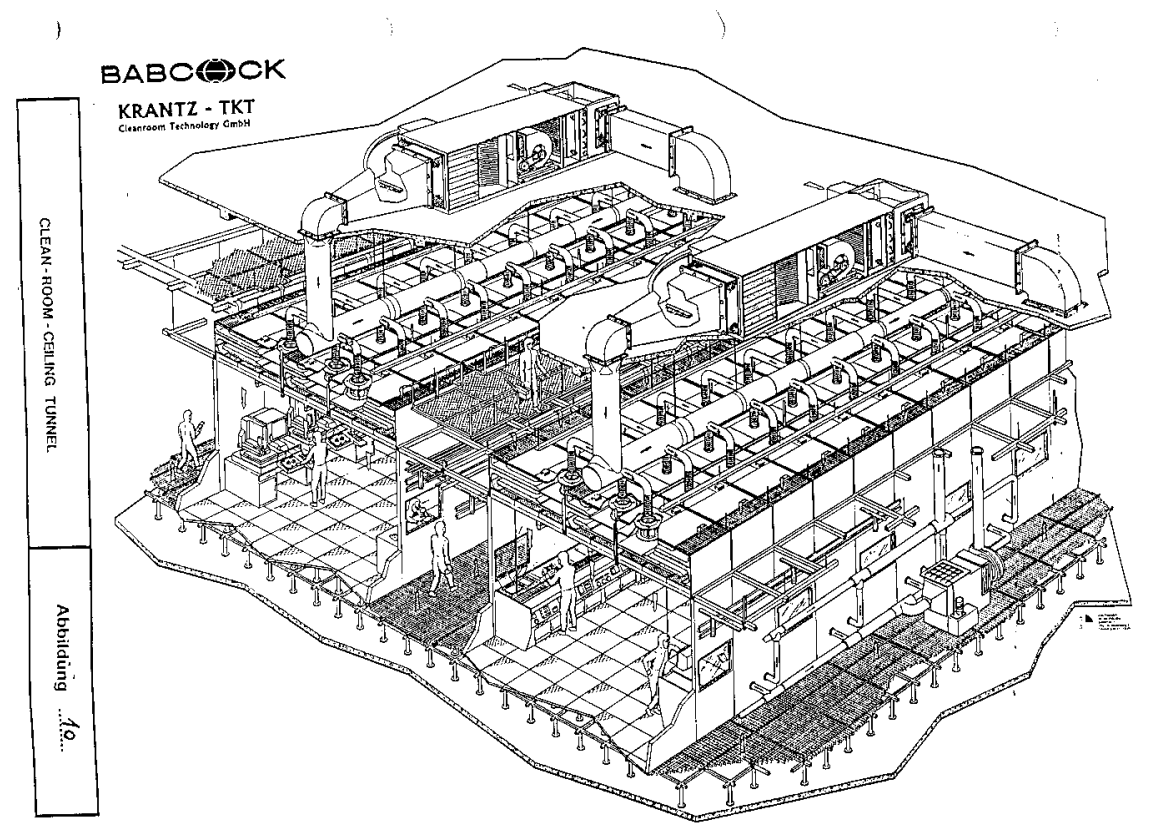

Figure 6. Clean room concept for RF cavity production.

Tesla Engineering Study Review 


\section{Input RF Couplers}

\section{a. Description}

Input power couplers are a particularly critical element of the cavity system. History has shown that they are technically high-risk components that can be as critical to a project as the cavities themselves. Couplers must perform to their power specification. They must be designed to be processable to operational level in a reasonable time. They must be able to be manufactured in a cost efficient method. Each coupler must deliver an instantaneous power of $232 \mathrm{~kW}$ (+ some control margin) over a $1.3 \mathrm{msec}$ pulse (including filling time) for TESLA 500 operation with single 9 cell cavities. This power level doubles for the $2 \times 9$ cell superstructure configuration. For $800 \mathrm{GeV}$ operation, the power is doubled from TESLA 500 levels. The input coupler for the superstructure would be required to operate at about $930 \mathrm{~kW}$. Couplers meeting these requirements would need to be installed initially.

The TESLA input coupler is a coaxial coupler mounted between the cryomodule outer vacuum shell and the cavity beam pipe. A series of prototype coupler designs have been evolving over the R\&D time. These design variants are discussed below. The coupler has two ceramic vacuum windows, one at room temperature and one at $70 \mathrm{o} \mathrm{K}$, in order to provide redundancy in case of window failure. A waveguide to coax transition takes place at room temperature. The present couplers have two bellows sections to provide the needed longitudinal (and transverse) motion during thermal cool down. The RF coupling to the cavity is adjustable.

With the 3rd generation cryostat design, couplers without longitudinal flexibility could be used. (See sections III.A.2.a.iii and 2.b.iii). These new couplers would still have transverse flexibility. The design would eliminate one coupler bellows, reduce coupler cost, and increase reliability. The planned coupler for TESLA will have fixed coupling.

Better pumping and heating helps cut down processing time. A plan under discussion is to improve $\mathrm{Al}-\mathrm{Mg}$ flange sealing gaskets for coupler to the cavity connection so baking can be done in situ to reduce coupler conditioning time. This might require protection of the cryomodule super-insulation as well.

Over the last three years, new codes have been developed by a Helsinki/DESY collaboration to understand multipacting zones in coaxial structures. New coupler designs have been developed to incorporate these advances in understanding and reducing multipacting. Later coupler designs incorporate the possibility of a DC bias on the antenna. It was shown at LEP and now at HERA and TTF that this is very helpful for reliable operation.

There has been a steady evolution of Input coupler design as indicated below and in the Table. This evolution has been driven by better understanding of the design needs (multipacting), schedule availability of RF windows, advances in the module design and fixed coupling that simplify the coupler, and the demands for higher power capability 
driven by $800 \mathrm{GeV}$ and the superstructure. Designs and procedures that minimize the coupler conditioning time as well as cost will be critical for production.

The table lists the impedance and diameter of the warm $\left(>70{ }^{\circ} \mathrm{K}\right)$ and cold $(4-70$ ${ }^{\circ} \mathrm{K}$ ) coupler sections. It lists the type window type and $\mathrm{TiN}$ coating for each section, whether coupling is adjustable and bias possible.

Fermi 1 - Eight of these couplers are installed in TTF module 2 (ACC2), five couplers in module $1^{*}$, and one coupler in the cavity for the injector (also one in the Fermilab A0 injector).. Over the past 2 years Module 2 had not been operated above 15 $\mathrm{MV} / \mathrm{m}$ due to operational requirements of the FEL. Presently the couplers limit the module gradient when operation is attempted above $15 \mathrm{MV} / \mathrm{m}$. It is believed that this is because of a combination of reasons related to the vacuum on the warm side of the coupler. This coupler is the first prototype, it does not have DC bias incorporated to suppress multipacting. The design had inadequate vacuum pumping conductance. The module has not been warmed up for considerable time (about 2 years) and it is believed that gasses evolving from the warm parts have condensed on the cold window. Whether a warmup and reconditioning of these couplers would restore their power capability has not been tested.

TTF II - These couplers are the first with a bias. Total of 16 installed in TTF modules 3 and 4 and three in module $1^{*}$. Module 3 (ACC1) is under long pulse test at 21-22 $\mathrm{MV} / \mathrm{m}$ with beam in spring of 2002. Two of these couplers have reached $1.8 \mathrm{MW}$ on the warm test stand and more units to $33 \mathrm{MV} / \mathrm{m}$ cold, sufficient for $800 \mathrm{GeV}$ single cavity operation.

TTF III - These are similar to TTF II, but have a less expensive cylindrical warm window. They are under production. Eighteen units have been tested warm to 1 MW.

TTF IV - for the 4-cavity * 7 cell type superstructure, $80 \mathrm{~mm} / 80 \mathrm{~mm}-70 \mathrm{ohm}$, bias. Two cylindrical windows, coupling fixed, with bias. There is more than one design. Two prototypes are under construction.

TTF V - for 2-cavity * 9 cell type superstructure. $60 \mathrm{~mm} / 60 \mathrm{~mm}$. RF design only.

\section{b. Coupler Testing}

The table provides status of test results.

Coupler testing is done with a room temperature test stand in traveling wave mode. In order to qualify at the field levels experienced in operation, the traveling wave power must be 4 times the operational power level. ( 4 MW for superstructure at 800 $\mathrm{GeV})$. 
Cold tests are also carried out with the coupler mounted to the cavity in horizontal test cryostat. Here the power requirement during cavity filling is as specified for module operation. Tests have been done at 500 microsec pulse duration, the fill time, and at 1.3 ms duration, the operating pulse length. It should be noted that at gradient without beam the RF flat top power level is $1 / 4$ that required for beam operation; during cavity fill time full power is supplied.

Additional short pulse testing (for High Peak Power processing) has been done up to $2 \mathrm{MW}$ and 500 microsec pulse duration. TTF 2 cavities have been tested up to $2 \mathrm{MW}$ for 200 microsec. The usual cold testing in Chechia is at $1 \mathrm{MW}$ for 500 microsec pulse duration. There has never been a test up to the final limit of the couplers; once the specified test level has been achieved, the testing is terminated.

\section{c. Coupler Conditioning}

Typical conditioning of a coupler takes 3-5 days (70 - $130 \mathrm{hrs)} \mathrm{warm} \mathrm{before}$ installation on a cavity. After coupler is mounted to a dressed cavity, conditioning in Chechia takes $\sim 1$ day cold. When the module is installed, low gradient operation at 400 $\mathrm{KW}$ occurs for 1-2 days, mostly for setting up the RF parameters, during which additional processing takes place. Normal operation at TTF is at $\sim 145 \mathrm{KW}$ power level. TiN coating of the entire coupler could significantly reduce processing time (down to 524 hours).

\section{d. Coupler Cost Study}

It is planned that there will be a separate fabrication and testing manufacturer for input couplers.

A coupler cost study for the TESLA TDR was conducted by Thomson under guidance from Orsay. Currently, for R\&D quantities, the RF power coupler costs are comparable to the cost for a bare cavity fabrication. In mass-production quantities for the cost estimate, it is believed that the coupler unit costs can be reduced by a large factor.

However, even in the $2 \times 9$ cell superstructure configuration, the coupler costs would still be projected to be a significant fraction of the sum of the costs for Niobium and cavity and vessel fabrication and assembly.

\section{e. Higher order mode couplers (HOM)}

It has been found that the orientation of one of the HOM couplers was incorrect to

damp a $2.585 \mathrm{GHz}$ mode. On future cavities the coupler will be mounted in the corrected configuration. This design change will not have impact on cost. 
TESLA Power Couplers (courtesy of W-D Möller):

\begin{tabular}{|c|c|c|c|c|c|c|c|}
\hline & Coupler type & FNAL & TTF I & TTF II & TTF III & TTF IV & TTF V \\
\hline cold & window & conical & cyl. & cyl. & cyl. & cyl. & cyl. \\
\hline & coax diameter, $\mathrm{mm}$ & 40 & 40 & 40 & 40 & 80 & 60 \\
\hline & Impedance, Ohm & 50 & 50 & 70 & 70 & 70 & 70 \\
\hline warm & window & flat, WG & cyl. & flat, WG & cyl. & cyl. & cyl. \\
\hline & coax diameter, mm & 60 & 40 & 60 & 60 & 80 & 60 \\
\hline & Impedance, Ohm & 50 & 50 & 50 & 50 & 70 & 50 \\
\hline coupli & ling & adjustable & adjustable & adjustable & adjustable & $\begin{array}{c}\text { not } \\
\text { adjustable }\end{array}$ & adjustable \\
\hline bias & & no & no & yes & yes & yes & yes \\
\hline Ceran & mic TiN coating & Fermilab & Fermilab & Fermilab & DESY & DESY & \\
\hline test & $2 \mathrm{~Hz} / 500 \mu \mathrm{s}$ & $1 \mathrm{MW}$ & $1 \mathrm{MW}$ & $2 \mathrm{MW}$ & $1 \mathrm{MW}$ & & \\
\hline & $2 \mathrm{~Hz} / 1.3 \mathrm{~ms}$ & $1 \mathrm{MW}$ & $1 \mathrm{MW}$ & $1.8 \mathrm{MW}$ & $1 \mathrm{MW}$ & & \\
\hline TW & Cold test done & yes & yes & no & no & no & \\
\hline horiz. & $2 \mathrm{~Hz} /<500 \mu \mathrm{s}$ & $1 \mathrm{MW}$ & $1 \mathrm{MW}$ & $1 \mathrm{MW}$ & $1 \mathrm{MW}$ & & \\
\hline & $10 \mathrm{~Hz} / 1.3 \mathrm{~ms}$ & $33 \mathrm{MV} / \mathrm{m}$ & $25 \mathrm{MV} / \mathrm{m}$ & $35 \mathrm{MV} / \mathrm{m}$ & $32 \mathrm{MV} / \mathrm{m}$ & & \\
\hline & cold test done & yes & yes & yes & yes & no & \\
\hline & fabricated total & 13 & 4 & 20 & $\begin{array}{c}22 \\
40 \text { ordered }\end{array}$ & 2 & \\
\hline & assembled to & Mod. $1^{*}, 2$ & Mod.1 & $\underset{4}{\operatorname{Mod} .1^{*}, 3}$ & $\begin{array}{c}\text { Mod.5, } 6 \\
(7,8)\end{array}$ & & \\
\hline
\end{tabular}

\section{f. Summary}

The input coupler is as critical a component as the cavity itself. Experience in TTF indicates that breakdowns in cryomodules almost always occurs at the coupler. There has been a steady evolution of the design, to make it more robust and able to handle higher power. However long term tests at high gradient and with long beam pulses are really needed to assess the long term operating reliability. This sort of systems qualification is not easy to fulfill and takes time.

Tesla Engineering Study Review 


\section{Cryomodules}

\section{a. Cryomodule Requirements}

The requirements for the cryomodules were established early in the TESLA program. The principal requirements are listed below. These have remained fairly constant over the life of the project. All the cryomodules built to date contain eight superconducting RF cavities plus a superconducting quadrupole and steering dipole set. This has been changed somewhat in the final design discussed in the TESLA Technical Design Report [1]. The major requirements of the cryomodules are outlined below.

\section{i. Alignment}

In order for TESLA to function properly as both a collider and a FEL driver, the cavities and magnets must be aligned to within certain tolerances. These tolerances must be maintained throughout transport, vacuum pumping and thermal cycling. For reasons of cost and complexity, there is no plan to allow adjustment of individual cavities once the module is assembled. Table 1 lists the alignment tolerances for the TESLA cryomodules. The axial tolerance is parallel to the accelerated beam while the transverse tolerance is in the plane perpendicular to the beam.

Table 1. Alignment tolerances for TESLA cryomodule.

\begin{tabular}{llll}
\hline Component & Transverse tolerance & Axial tolerance & Rotational tolerance \\
\hline Cavity & $+/-0.5 \mathrm{~mm}$ & $+/-5 \mathrm{~mm}$ & - \\
\hline Quadrupole & $+/-0.2 \mathrm{~mm}$ & $+/-5 \mathrm{~mm}$ & $+/-0.1 \mathrm{mrad}$ \\
\hline
\end{tabular}

It is not clear (at least in our discussions) if the alignment specifications have been completely analyzed in terms of the beam dynamics requirements, or that the understanding of the specification is common between the dynamics people and the cryomodule people.

A few questions are: how is the cavity centerline determined and how good is it?, how critical is the tilt of a cavity with respect to the beam direction?, what sort of distribution of errors is assumed by both the beam dynamics people and the cryomodule people?, what are the specifications for systematic or semi-systematic errors like module alignment?, how are alignment tolerances budgeted to different sources?, what is the expected tunnel motion with time and will it be necessary to realign modules? We believe this is an area where further clarification and effort would be very worthwhile.

\section{ii. Static Heat Leak}

As TESLA is a superconducting RF device, the majority of the cryogenic load comes from the RF power. The TESLA application is the first pulsed superconducting RF accelerator idea. A basic underlying assumption in the design is that total electrical Tesla Engineering Study Review 
power usage be reasonable. This along with expected cavity $\mathrm{Q}$ sets reasonable bounds on RF pulse duty factor. In turn this leads to a criterion for the desired static leak- that it be a fraction of the dynamic load. The size of TESLA dictates that the static heat leak into the cryomodules be kept as small as reasonable. This has made for an engineering challenge that has not been as important in smaller installations to date. Table 2 gives the calculated static heat leaks at the $2{ }^{\circ} \mathrm{K}, 4.5^{\circ} \mathrm{K}$ and $70{ }^{\circ} \mathrm{K}$ levels. These calculated loads then became part of the integrated cryogenic system specifications.

Table 2. Predicted TTF and TESLA cryomodule heat leaks, not including ends and feed cap contributions

\begin{tabular}{lcll}
\hline $\begin{array}{l}\text { Temperature } \\
\text { Level }\end{array}$ & $\begin{array}{c}\text { TTF }-\mathbf{1 2} \mathbf{~ m} \\
\text { static }- \text { ref } 2 \\
\mathbf{1}^{\text {st }} \text { generation } \\
\text { cryomodule }\end{array}$ & $\begin{array}{l}\text { TESLA }-\mathbf{1 7} \mathbf{~ m} \\
\text { static }- \text { TDR } \\
\mathbf{3}^{\text {rd }} \text { generation } \\
\text { cryomodule }\end{array}$ & $\begin{array}{l}\text { TESLA }-\mathbf{1 7} \mathbf{~ m} \\
\text { dynamic }- \text { TDR } \\
\mathbf{2 3 . 4} \mathbf{~ M V} / \mathbf{m} . \mathbf{Q}_{\mathbf{0}}=\mathbf{1 0} \mathbf{1 0}^{\mathbf{1 0}}, \mathbf{5} \mathbf{~ H z} \\
\text { high energy beam }\end{array}$ \\
\hline $70^{\circ} \mathrm{K}$ & 76.8 watts & 90.1 watts & 93 watts \\
\hline $4.5^{\circ} \mathrm{K}$ & 13.9 watts & 11.3 watts & 4.6 watts \\
\hline $2^{\circ} \mathrm{K}$ & 2.8 watts & 1.74 watts & 7.3 watts \\
\hline
\end{tabular}

\section{iii. Movable Couplers}

The fixed point of the cryomodule is at the center. Thus, the ends of the $12 \mathrm{~m}$ long module move $15 \mathrm{~mm}$ towards the center during cool down. This leaves the designer with a choice. Either fix the power couplers (and thus the cavities) with respect to the $300 \mathrm{~K}$ vacuum vessel and let them move relative to the rest of the cold mass, or fix them to the cold mass and design the coupler to move relative to the $300 \mathrm{~K}$ vacuum vessel. Early on in the TESLA project it was decided to take the second option (that of movable couplers). Couplers have been designed to meet these requirements. It may be preferable to use fixed couplers and the third generation cryomodule design permits the use of movable (and of course fixed) couplers.

\section{iv. Vibration}

Excessive vibration can affect the cavity tuning and resulting beam performance. The cryomodule should be designed to reduce the vibration to the cavities and magnets. The resonant frequencies of the cryomodule should be such as to avoid resonant excitation at the 5- $\mathrm{f} 10 \mathrm{~Hz}$ repetition rate of the accelerator.

\section{v. Cost}

The size of TESLA dictates that the cost of each cryomodule must be kept as low as possible. While there is not a specific goal, reducing the cost of the cryomodule has Tesla Engineering Study Review 
been a consideration from the beginning. In particular, it was desired to have the cost of the cryomodules to be significantly less than that of the CEBAF cryomodules. This has lead to the design of long cryomodules to minimize the number of expensive interconnects. Cost was the principal reason for changing the thermal shield design between cryomodule \#1 and cryomodule \#2.

\section{b. Evolution of Cryomodule Design}

To date, three generations of TESLA cryomodules have been designed and built. In each case, the design changes were motivated by a desire to reduce cost and improve performance. Data from tests of completed cryomodules in the TTF Linac and experience from the assembly of cryomodules have been a major factor in the design changes.

\section{i. $1^{\text {st }}$ Generation Cryomodule}

A cross section of the first generation design $[3,4,5]$ is shown in Figure 1 . The cryomodule is $12 \mathrm{~m}$ long and contains 8 superconducting RF cavities. It also contains a superconducting quadrupole, steering dipole, and beam position monitor package. The cavities are bath cooled by saturated He II at $2 \mathrm{~K}$. The cavity baths are supplied by a parallel two-phase He II line. The heat deposited in the He II evaporates vapor at the surface of the two-phase line and the resulting helium gas is returned to the refrigerator via a large diameter $(300 \mathrm{~mm})$ gas return pipe, resulting in a pressure at the cavities of less than 31 mbar. The gas return pipe and two-phase line are connected together at the end of each module. The superconducting magnets are cooled by a separate $4.5 \mathrm{~K}$ flow. The same flow also cools the $4.5 \mathrm{~K}$ thermal shields. A second set of shields is cooled by a separate $70 \mathrm{~K}$ flow. The cryomodule also contains multilayer superinsulation (MLI) blankets on the $4.5 \mathrm{~K}$ and $70 \mathrm{~K}$ thermal shields and a separate warm up / cool down pipe parallel to the cavity string. All process lines are contained within the cryomodule.

The outer diameter of the vacuum vessel pipe is approximately $1.1 \mathrm{~m}$. The cavities and quadrupole package are directly attached to the $300 \mathrm{~mm}$ gas return pipe. This pipe is itself attached to the 3 composite support posts arranged axially along the length of the module. The middle post is fixed. The 2 outer posts along with the $300 \mathrm{~mm}$ pipe, cavities, magnets, and shields - all move toward the center of the cryomodule as a result of thermal contraction during cool down.

The gas return pipe is the structural backbone of the cryomodule and is key to the alignment of the cavities and quadrupole. The gas return pipe is aligned relative to the ideal beam axis via adjusting screws on the support posts. The cavities and quadrupole are then each aligned relative to the ideal beam axis via adjustment screws attaching them to the $300 \mathrm{~mm}$ pipe. Once this is accomplished, the cavity alignment is determined by the pipe alignment. As long as the gas return pipe is in the proper position relative to the beam axis, the cavities will be as well. This design only works properly if the cavities don't move relative to the $300 \mathrm{~mm}$ pipe once aligned, and the $300 \mathrm{~mm}$ pipe doesn't move in an unexpected manner once aligned relative to the beam axis. Upon cooling, thermal 
contraction will cause the $300 \mathrm{~mm}$ pipe, cavities and quadrupole to move vertically upwards by $1.8 \mathrm{~mm}$ relative to their warm position. This effect is allowed for in the alignment process.

In the first generation cryomodule, the thermal radiation shields at $4.5 \mathrm{~K}$ and $70 \mathrm{~K}$ are constructed from aluminum and the cooling pipes are stainless steel. Large copper braids fastened between the shields and the cooling pipes cool the shields. Each thermal shield is divided into a top piece and a number of bottom sections connected together by threaded fasteners.

One of these $1^{\text {st }}$ generation cryomodules was assembled in 1997. The assembly time was 10 weeks, after some time of initial preparation and modification.

This cryomodule was extensively tested in the TTF Linac $[3,4,5,6]$. The salient results were:

- The static heat leak was somewhat higher than predicted but the results indicated that this was due to the large number of additional instrumentation cables used in this cryomodule.

- The $300 \mathrm{~mm}$ pipe deforms due to unbalanced vacuum loading. The result of this is that while the cavities stay within their alignment tolerance the quadrupole which is at the end of the module moves outside its alignment tolerance.

- No vibration problems were seen with the cavity performance.

Considerable effort went into straightening the $300 \mathrm{~mm}$ pipe. An unbalanced vacuum load was caused by errors in the relative positions of the pipe ends. Experience has shown that the effort on straightening the pipe was not cost effective and a better solution was found in the $3^{\text {rd }}$ generation design (see below). 


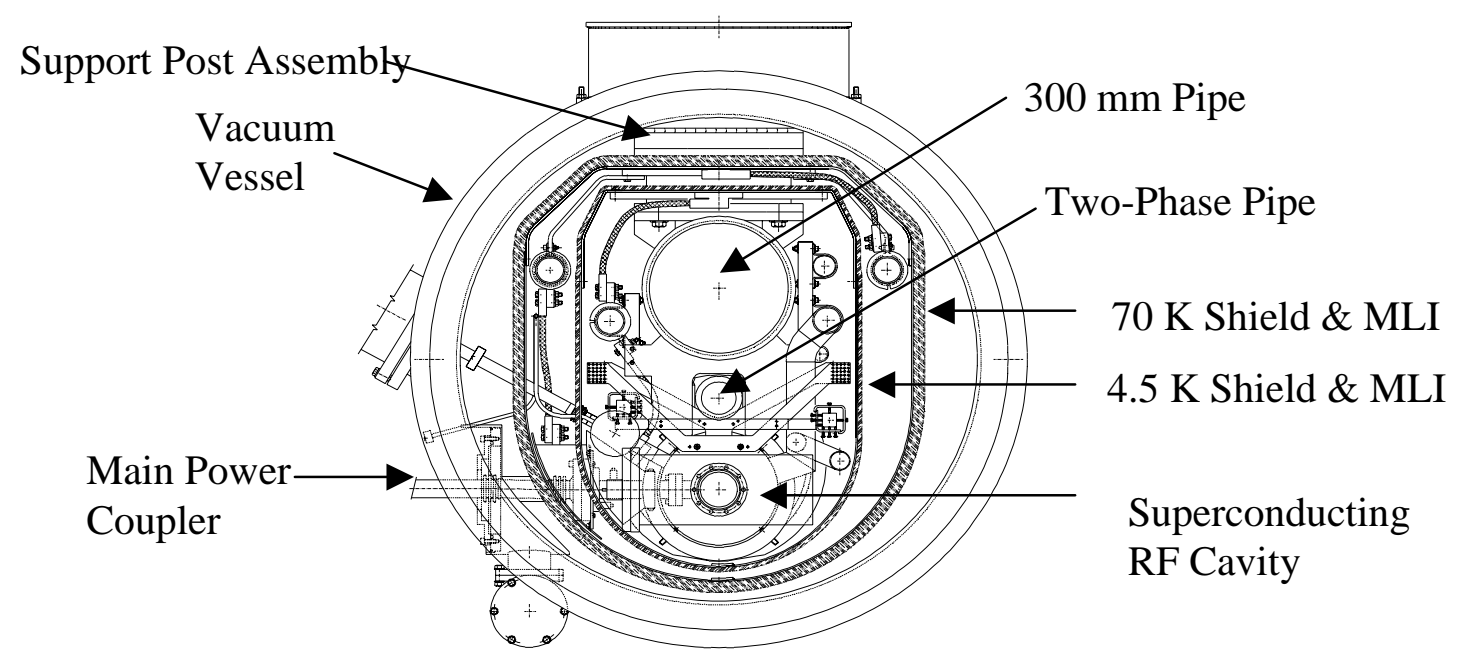

Figure 1. Cross Section of $\mathbf{1}^{\text {st }}$ Generation Cryomodule.

\section{ii. $2^{\text {nd }}$ Generation Cryomodule}

The $2^{\text {nd }}$ generation design was started even before the $1^{\text {st }}$ generation design cryomodule was tested. This design is very similar to the 1 st generation design with modifications to the thermal radiation shields. There were two problems with the initial shield design. First, the copper braids used to cool the shields were bulky, expensive and not completely reliable. Second, the use of threaded fasteners to connect the shield pieces together was very expensive in both material and manpower. To solve these problems, it was decided to cool the shields with aluminum cooling pipes that were directly welded to the shields. This sort of assembly is similar to that used for the shield designs for LHC and SSC superconducting magnets. Welding was also used instead of fasteners to connect the shield pieces together. In order to prevent excessive stress and deformation of the welded joints during thermal cycling a finger welding technique was implemented. Extensive finite element analysis modeling was performed [7] to predict the behavior of the new shield design. The use of welded shields does place limits on the cryomodule cool down rate, but these limits are consistent with those imposed to prevent excess deformation of the gas return pipe during cool down.

Based on the experience of cryomodule \#1, the mechanical tolerances of the second prototype design were adjusted. Those tolerances that were tighter than necessary were relaxed and the reference points of all the tolerances were adjusted to better match the manufacturing and assembly process.

The changes to the thermal shield design and the tolerances resulted in a cost savings of $45 \%$ for the cold mass (that is everything except the cavities, quadrupole and their related components) between the first- and second-generation designs. Two Tesla Engineering Study Review 
cryomodules (cryomodules \#2 and \#3) were built to this $2^{\text {nd }}$ generation design. The assembly time for these two cryomodules was 7 weeks each. Both cryomodules \#2 and \#3 have been tested $[3,4,5,6]$ in the TTF Linac. The results were very similar to those from the first generation cryomodule. Table 3 shows the static heat leak measurements for both cryomodule \#1 and \#2. Feed and end caps were measured separately from the modules. Note that the heat leak results for cryomodule \#2 are much closer to the predicted values than those of cryomodule \#1. This results from far less instrumentation and thus fewer instrumentation cables between room and cryogenic temperatures. It also reflects a learning curve with improved installation for better thermal insulation during assembly. The new thermal shield design also worked as expected. The temperature distributions in the shields were as predicted and no evidence of mechanical damage to the shields was seen upon cool down.

The Qo of each cavity is measured in the vertical dewar test as a function of gradient. After the input coupler has been mounted, Qo can no longer be measured directly because the external Q is so much lower than Qo. When a cavity is tested in Chechia (horizontal test dewar) or after it is in a module, the dynamic cavity heat load from RF losses can be measured as a function of gradient. These measurements are used to determine the cavity Qo as assembled in the module. Thus if a cavity Qo had deteriorated during helium vessel, input coupler, and module assembly, it would be apparent from the cryogenic measurements.

Table 3. Static heat leak results.

\begin{tabular}{|c|c|c|c|c|}
\hline $\begin{array}{l}\text { Temperature } \\
\text { Level }\end{array}$ & $\begin{array}{l}\text { Predicted Heat } \\
\text { Leak }(W) \\
\text { Ref. } 2\end{array}$ & $\begin{array}{l}\text { Measured Heat } \\
\text { Leak (W) } \\
\text { Cryomodule \#1 } \\
\text { (alone) }\end{array}$ & $\begin{array}{l}\text { Measured Heat } \\
\text { Leak (W) } \\
\text { Cryomodule \#1 } \\
\text { (with \#2) } \\
\text { after repair }\end{array}$ & $\begin{array}{l}\text { Measured Heat } \\
\text { Leak (W) } \\
\text { Cryomodule \#2 } \\
\text { (with \#1) }\end{array}$ \\
\hline $70^{\circ} \mathrm{K}$ & 76.8 & 90 & 81.5 & 77.9 \\
\hline $4.5^{\circ} \mathrm{K}$ & 13.9 & 23 & 15.9 & 13 \\
\hline $2{ }^{\circ} \mathrm{K}$ & 2.8 & 6 & 5 & 4 \\
\hline
\end{tabular}




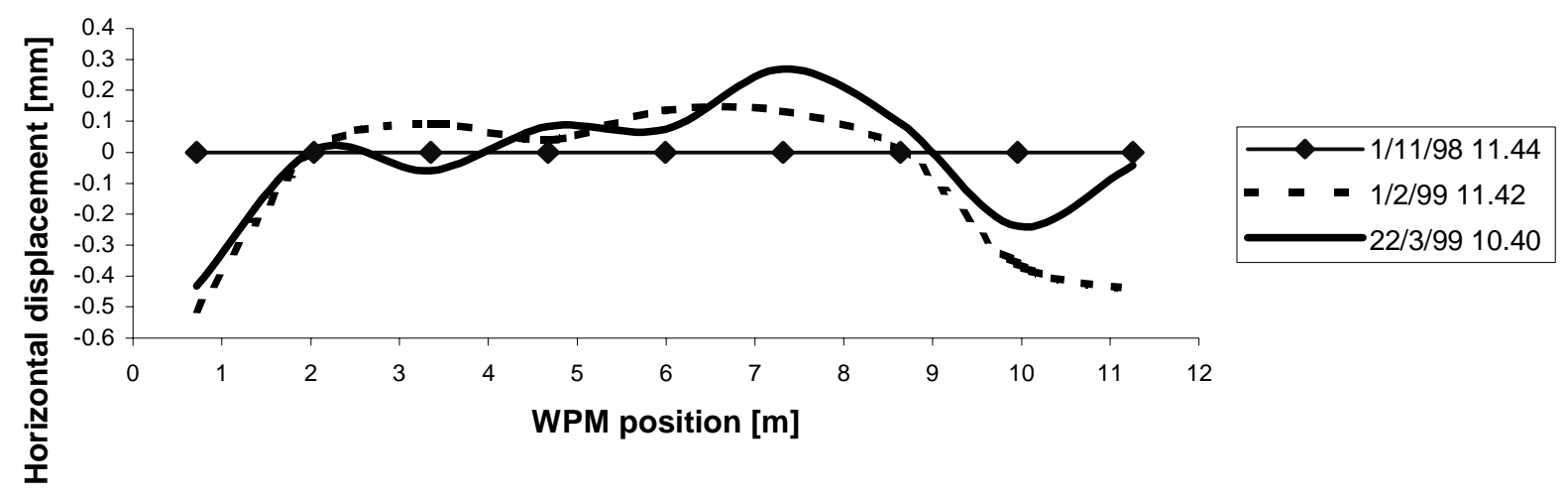

Figure 2. Horizontal displacements of the cavity string for Cryomodule \#2 during cooldown (1/2/99) and warmup (22/3/99) compared to the original position (1/11/98).

\section{iii. $3^{\text {rd }}$ Generation Cryomodule}

Based on the results from the first two generations of cryomodule design, a third generation design has been developed. This new design:

- Reduces the outer diameter of the cryomodule from $1.1 \mathrm{~m}$ to standard 38". This was accomplished by changing the connection of the cavities to the cold mass and by slightly moving the two-phase pipe. The smaller size frees up room in the proposed TESLA tunnel. It also allows the use of a standard pipe size for the vacuum vessel, which results in further cost savings. In the original design a larger diameter cavity helium vessel had been planned for. It was found that this diameter was not necessary and was never used. The $3^{\text {rd }}$ generation module is the first to make use of the space saved by the smaller vessel.

- Moves one of the posts so that the quadrupole magnet is directly under it to minimize deflection of the $300 \mathrm{~mm}$ tube. Calculations show that this will allow the quadrupole to meet its tight alignment tolerances

- Allows the use of rigid or semi-rigid main couplers by connecting the cavities to the cold mass by a series of roller bearings and a parallel Invar rod. This may result in significant cost savings for the main coupler.

- In the previous cryomodule generations, the attachment points between the cavities and the $300 \mathrm{~mm}$ pipe were rotated 45 degrees from the vertical and horizontal planes. This made alignment of the cavities difficult. In the $3^{\text {rd }}$ generation cryomodule, the attachment points are in the vertical and horizontal planes. This makes cavity alignment much faster.

- Uses the improved thermal shield design tested in cryomodules \#2 and \#3.

In this generation, rather than taking great efforts to straighten the pipe, a pipe with standard industrial tolerances is used. It is mounted on a large numerically controlled mill, measured, and then the connections to the support posts, the ends of the 
pipe, and the connections to the cavity string are all machined at once. This results in a superior positioning of the pipe ends relative to the supports and to the other modules.

Figure 3 shows a cross sectional view of the new design. Further details of this design may be found in reference [8]. Three of these new cryomodules have been built. Due to improved assembly procedures and the new location of the attachment points, the assembly time for this module was only 3 weeks. Due to the different size of the vacuum vessel a $3^{\text {rd }}$ generation cryomodule has not been tested yet in the TTF Linac.

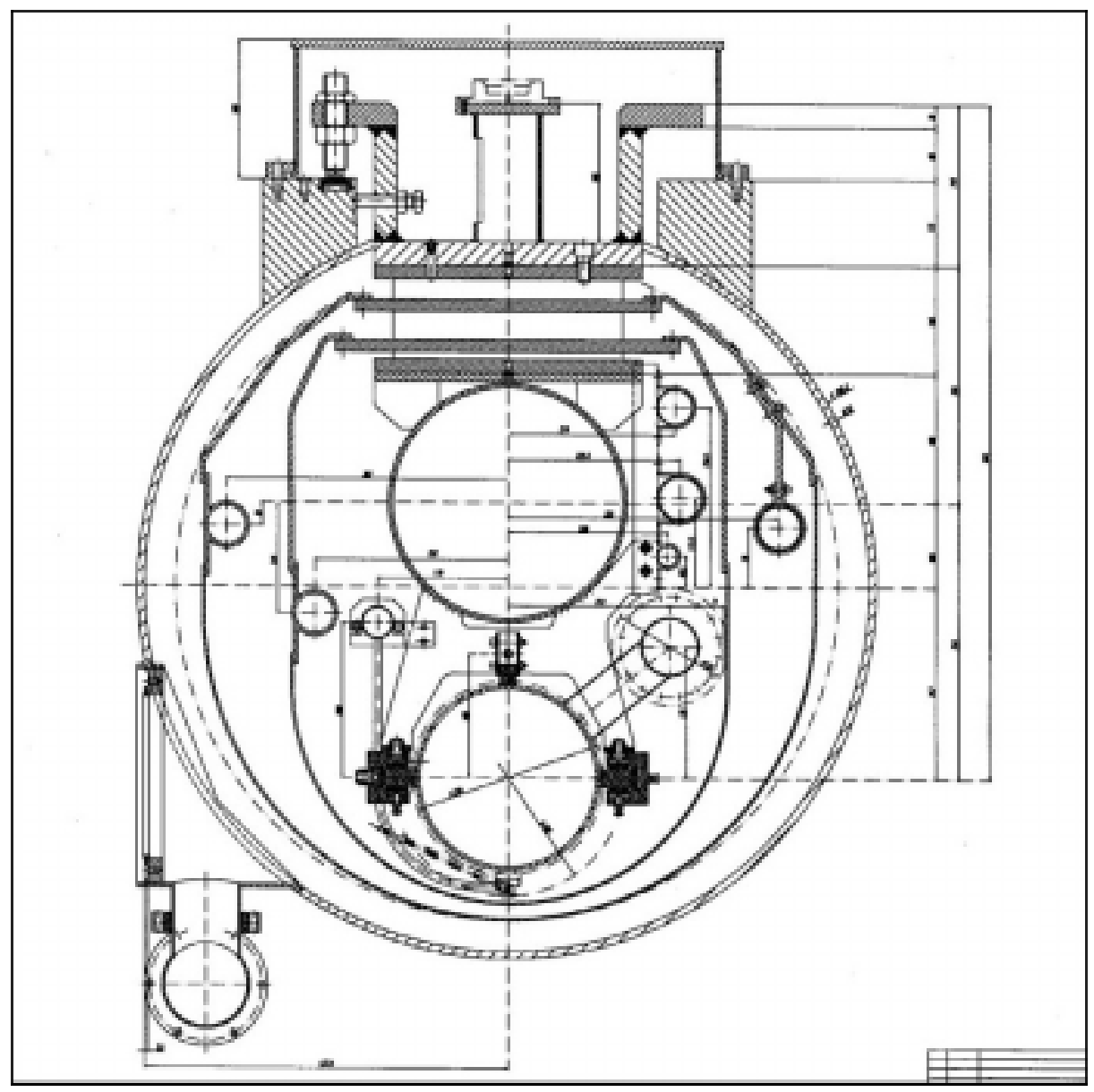

Figure 3. Cross Section of the $3^{\text {rd }}$ Generation TESLA Cryomodule

\section{iv. Fermilab Participation in Cryomodule Design}

Fermilab was involved in the design of the early cryomodules almost from the beginning. We participated in most of the collaboration meetings held from 1991 through 1993 and were active in the cryostat working group sessions. We developed several design alternates; most of our design input involved the suspension system and input coupler designs. We also encouraged the development of an input coupler without the flexibility of those in initial designs. Much design and analysis work was done to 
quantify the structural response of the suspension system when subject to shipping and handling loads and during cooldown. We proposed the development of a cryostat with the supports mounted on the bottom as a means of minimizing the cold mass deflection during transport [9].

The analysis of what modifications will be required to the present module assembly to allow for transport and shipping without damage or misalignment still needs to be thought through. This is an ideal area for future collaboration.

\section{v. Final Design}

The baseline cryomodule design is well documented in the Technical Design Report and doesn't need to be detailed here. It is largely based on the third generation design developed jointly between INFN-Milan and DESY. It consists of a 17-meter long vacuum vessel housing twelve 9-cell cavities, a quadrupole, steering dipole, and beam position monitor. The effective accelerating length has increased from earlier designs as a means of increasing the packing factor - the number of accelerating structures per unit length of linac, and reducing the number of interconnects, valves, bellows, etc. Another difference is the vacuum vessel diameter that has been decreased to a standard diameter pipe of 38 inches. The quadrupole and its integral steering dipole and beam position monitor has been moved to the center of the structure immediately adjacent to the center support as a means of increasing its structural support and long-term stability. Lastly, the quadrupole in the final design is cooled by the $2{ }^{\circ} \mathrm{K}$ rather than the $4{ }^{\circ} \mathrm{K}$ circuit.

Although these features constitute part of the baseline design, there is still some debate about several key elements of the design. For example, although the desire is for the longer 17-meter long modules mentioned above, there is still some interest in maintaining the current 12-meter length. The shorter modules are more compatible with existing machining equipment, may facilitate shipping, and allow for a smaller shaft diameter. There is also an effort underway to develop cavity superstructures - two cavities fabricated as a single unit to utilize a single input coupler. It is probable that whatever technology works at the time of industrial participation should be the design used in production, even if it is the current, individual cavity configuration. 


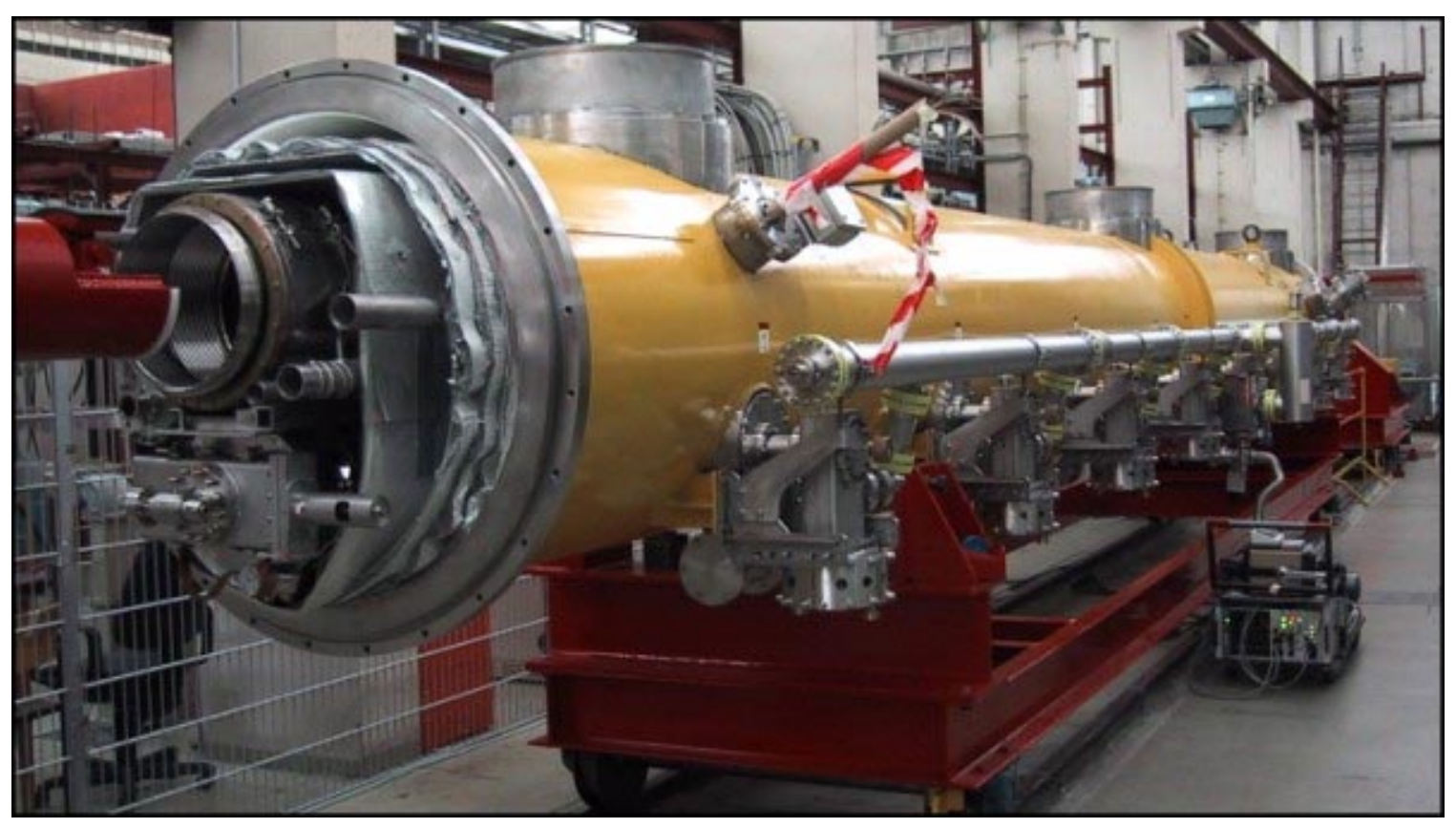

Figure 5. Completed final design cryomodule (12-meter long version)

\section{c. Cryomodule Review and Industrial Studies}

In 1999, an external panel conducted a review of the cryomodule design. The panel was chaired by Ph. Lebrun of CERN and also included O. Groebner of CERN and P. Kelley of LANL. The committee was impressed with the work done to date and could not identify any major problems that would prevent the cryomodule design from meeting its requirements. They did suggest that the quadrupole be placed in the center of the cryomodule to insure that it stays within its alignment tolerance and that vacuum barriers be added along the length of the cryomodule string to facilitate leak checking and repair. Both of these suggestions have since been included in the final design described in the TESLA TDR.

The committee also suggested that the TESLA collaboration should consider how the TESLA cryomodule design fits into the overall operation of the TESLA machine. The committee felt that analysis should be done on the impact of the cryomodule design on overall machine availability, reliability and the identification of failure modes. The committee was not able to point to any specific problems but felt that more work was needed. The TESLA TDR does explain how the cryomodule fits in with other subsystems in the TESLA machine but additional work on failure mode analysis, availability and reliability still needs to be done.

DESY also commissioned two independent industrial studies of cavity treatment and cryomodule assembly. These studies were carried out by the firms Accel and Noell. The studies lasted more than six months and involved a significant review of the designs, interviews with DESY staff and surveys of industry. The results of the studies included Tesla Engineering Study Review 
the facilities and process for producing completed cryomodules along with the cost of doing so. Since two industrial studies were done, DESY have two sets of cost numbers available. While these numbers were not greatly different from each other it is important to note that the TESLA collaboration took the higher number for use in the TDR cost estimate.

\section{d. Cryomodule Cost Study}

The $€ 1.13$ B estimate for the Main Linac Modules is for the off-site fabrication by industrial companies. It does not include any cavity or module testing at DESY or post-fabrication work at DESY, transportation, installation, commissioning, etc. TESLA Collaboration manpower for these tasks is included in the manpower estimate.

The major cost items that went into the overall cryomodule cost study include: naked cavities, including HOM couplers; cavity preparation; RF power couplers; vacuum vessel, shields, insulation, etc., including cryogenic valves, actuators, and instrumentation; tuner structure, gearboxes, motor, and electronics; titanium vessels, piping, and bellows; piezo tuner, quadrupole magnet package with beam position monitor; instrumentation; magnetic shielding material and fabrication; module interconnections and beam vacuum; module and end supports; and power supplies for magnets. However installation of modules ( 178 man-years est.) and installation of diagnostics ( $\sim 6$ man-years est.) are not included in the total cryomodule cost estimate.

\section{e. Concluding Remarks on Cryomodules}

The current design of the TESLA cryomodule is the result of an ongoing design evolution based on both actual tests of the cryomodules in the TESLA Test Facility and design reviews. Previous testing shows that the TESLA cryomodule described in the TDR should meet all its design requirements. Additional work on failure mode analysis and impact of cryomodule reliability on machine availability should be carried out. While the TESLA cost estimate does not have an explicit contingency in costs, the cost numbers used are somewhat conservative and not the lowest costs quoted. Continued $\mathrm{R} \& \mathrm{D}$ in cost reduction or lower bids could generate a contingency "margin".

In addition to these items and fine tuning the cost study included in the TDR, some effort should be made to determine how best to structure the collaboration to bring the expertise of all the involved organizations to bear as effectively as possible. Some potential areas of thought are development of U.S. suppliers of vacuum vessels, cavities, support structures, etc., continued R\&D into ways of further reducing costs, and continued involvement of laboratories outside of DESY in the detailed design process. 


\section{f. Cryomodule References}

(1) TESLA Technical Design Report, DESY 2001-011 (March 2001).

(2) TESLA Test Facility Linac - Design Report, Version 1.0, March 1995, (TESLA 95-01), Table 5.4.

(3) "Design, manufacture, and test of the TESLA-TTF cavity cryostat", F. Alessandria, G. Cavallari, M. Minestrini, T.H. Nicol, C. Pagani, R. Palmieri, S. Tazzari, and G. Varisco, Advances in Cryogenic Engineering, Vol. 41a, p. 825 (1996).

(4) "Construction, Commissioning and Cryogenic Performance of the First TESLA Test Facility (TTF) Cryomodule", C. Pagani, J.G. Weisend II, R. Bandelmann, D. Barni, A. Bosotti, G. Grygiel, H. Kaiser, U. Knopf, R. Lange, F. Loeffler, R. Pierini, O. Peters, B. Petersen, D. Sellman, and S. Wolff, Advances in Cryogenic Engineering, Vol. 43a, pp. 87 (1998).

(5) "The TESLA Test Facility (TTF) Cryomodule: A Summary of Work To Date", J.G. Weisend II, C. Pagani, R. Bandelmann, D. Barni, A. Bosotti, G. Grygiel, R. Lange, P. Pierini, B. Petersen, D. Sellmann, S. Wolff, Advances in Cryogenic Engineering, Vol. 45a, pp. 825-833 (2000).

(6) "Operating Experience with the First TESLA Test Facility (TTF) Cryomodule", J. G. Weisend II, R. Bandelmann, D. Barni, A. Bosotti, G. Grygiel, H. Kaiser, U. Knopf, R. Lange, F. Loeffler, C. Pagani, P. Pierini, O. Peters, B. Petersen, D. Sellmann, S. Wolff, Proc. ICEC 17, 601 ( 1998 ).

(7) " Design of the Thermal Shields for the New Improved Version of the TESLA Test Facility ( TTF ) Cryostat ", C. Pagani, D.Barni, M. Bonezzi, P. Pierini, J. G. Weisend II, Advances in Cryogenic Engineering, Vol. 43a, pp. 307 ( 1998 ).

(8) "Further Improvements of the TESLA Test Facility (TTF) Cryostat in View of the TESLA Collider", C. Pagani, D. Barni, M. Bonezzi and J. G. Weisend II, Advances in Cryogenic Engineering, Vol. 45A, pp. 939-946 (2000).

(9) “TESLA Test Facility Alternate Cryostat Design", T.H. Nicol, Advances in Cryogenic Engineering, Vol. 41a, pp. 839, Plenum Press, New York, 1995. 


\section{Concluding Remarks on Main LINAC Modules}

\section{a. Component comparisons and optimization}

The cavity and cryomodule

The TESLA cost estimate for a complete 17 meter module is $1131 / 1716=660 \mathrm{~K}$ Euros (or $\sim 450 \mathrm{~K}$ Euros for 12 meter modules). These include cavity processing and assembly labor, but not cavity or module test labor, to be done at DESY.

The DESY labor for TTF is difficult to estimate. The preparation labor for each cavity is about 185 man-hours prior to assembly into a string (this includes chemistry, tuning, and oven bake out. Multiplying by 8 cavities, this gives 185 man-days per cryomodule for this cavity prep work. The assembly of the TTF cavity string takes about 15 man-days. The assembly of the string into the module takes approximately 60-80 man-days (see below). This totals $\sim 270$ man-days $\sim 1.1$ man-year $=55 \mathrm{~K}$ Euros.

Present cost of an 8 cavity, 12 meter long TTF cryomodule is approximately 1-1.1 M Euros, including labor. Thus it appears that the overall unit cost for the TESLA production run will be $\sim 40 \%$ of that for comparable TTF cryomodules (scaling between 8 and 12 cavity configurations), including a rough estimate for labor.

Although it is difficult to directly compare costs for different technologies (frequency, configuration, cryogenics distribution, mechanical supports, etc.) Table 1 presents a listing of the costs for cryomodules for SCRF accelerators. The TTF costs are our best understanding, not carefully researched numbers. More details for CEBAF and SNS are available in the Appendix.

This comparison shows that the actual or projected cost of $1 \mathrm{MV}$ of accelerating gradient (integrated over cryomodule length) is \$ $41 \mathrm{~K}$ for CEBAF (5 MV/m), \$ $29 \mathrm{~K}$ for SNS hi- $\beta(12.6 \mathrm{MV} / \mathrm{m}), 5 \mathrm{~K}$ Euros + labor for TTF $(25 \mathrm{MV} / \mathrm{m})$, and $\sim 2 \mathrm{~K}$ Euros for TESLA $(25 \mathrm{MV} / \mathrm{m})$. This demonstrates the decrease in cost as cavity performance increases, the continued optimization and simplification of the cryostat and cryogenic distribution systems for TTF/TESLA, and the cost reductions due to industrial mass production for the evolution from TTF to TESLA quantities. It is also noted that the associated tunnel length and its cost decreases with increasing accelerating gradient. One should note that CEBAF is $\mathrm{CW}$, so high gradient operation is limited by cryogenic losses. Gradients at CEBAF have increased since initial operation to $\sim 7-8 \mathrm{MV} / \mathrm{m}$. SNS is considerably lower frequency, consequently the cavities and modules are bigger. 
Table 1. Comparison of SC RF Cryomodule Costs - in units of \$ $1 \mathrm{~K}=1 \mathrm{~K}$ Euros

\begin{tabular}{|c|c|c|c|c|}
\hline Item & TTF* & $\underline{\text { TESLA }}$ & CEBAF $^{* *}$ & $\underline{\text { SNS hi- } \beta}^{* *}$ \\
\hline frequency - MHz & 1300 & 1300 & 1500 & 805 \\
\hline \# cells per cavity & 9 & 9 & 5 & 6 \\
\hline \# cavities per cryomodule & 8 & 12 & 8 & 4 \\
\hline $\mathrm{E}_{\mathrm{acc}}(\mathrm{MV} / \mathrm{m})$ & 25 & 25 & 5 & 12.6 \\
\hline $\mathrm{L}_{\text {cavity }}$ (meter) & 1.04 & 1.04 & 0.5 & 0.91 \\
\hline $\mathrm{E}_{\text {acc }} \mathrm{L}_{\text {cavity }}$ (MV/cryomodule) & 208 & 312 & 20 & 47 \\
\hline \# (to be) produced & few & 1,716 & 43 & 12 \\
\hline date of estimate/cost & 2001 & 2000 & 1991 & 2002 \\
\hline cavity assemblies & & & & \\
\hline niobium & 136 & & 48 & 578 \\
\hline cavity fabrication & 264 & & 240 & sum \\
\hline helium vessel & 56 & & 32 & 90 \\
\hline input couplers & 240 & & 13 & - \\
\hline tuners & 40 & & 22 & 75 \\
\hline miscellaneous & 40 & & 23 & 6 \\
\hline HOM loads & & & 13 & \\
\hline cold windows & & & 20 & \\
\hline pair parts & & & 56 & \\
\hline top hats & 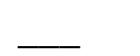 & & 26 & \\
\hline sum for dressed cavities & 776 & & 492 & 750 \\
\hline magnets & 30 & & - & - \\
\hline $\begin{array}{l}\text { cryo vessel including } \\
\text { helium headers, } \\
\text { shields, end cans, } \\
\text { mech. supports }\end{array}$ & 190 & & 131 & 327 \\
\hline labor & 55 & incl. & $\underline{200}$ & $\underline{294}$ \\
\hline Total Cryomodule cost & 1051 & 660 & 823 & 1,371 \\
\hline Cost per active meter & 126 & 53 & 206 & 377 \\
\hline Cost ( $\$$ K or K Euros/MV) & 5.2 & 2.1 & 41 & 30 \\
\hline
\end{tabular}




\section{The bare cavity}

Presently the cavity costs $\sim 50 \mathrm{~K}$ Euros. $2 / 3$ of the cost is in fabrication. $1 / 3$ in $\mathrm{Nb}$ material. The cost of fabrication of the "end groups" (end 1/2cells, beam tubes, couplers, flanges, etc is approximately equal to the cost of fabrication of the cavity body. For this reason, and because of the necessity for stiffening rings, fabrication by spinning or hydroforming may not result in a great cost savings.

TTF costs of cavity fabrication are dominated by the e-beam welding and prep for welding (50\%) and by machining of the cups (36\%) after they have been drawn.

\section{Niobium}

Present cost of $\mathrm{Nb}$ is about 50 Euros $/ \mathrm{kg}$ before refining and $\sim 500 \mathrm{E} / \mathrm{kg}$ for RRR300 after refining. The amount of $\mathrm{Nb}$ needed for production (500 tons) is not significant on the scale of world usage. However the purification process needs to be improved for cost reduction. It is proposed to have a special dedicated furnace for the $3 \mathrm{rd}$ melt (that would not be used for NbTi). This should reduce the number of melting cycles required.

Other cost saving measures include: band rolling instead of plate rolling as is done now (however there is some indication that band rolling may be causing problems at SNS), making one good side free of imperfections, having the supplier perform eddy current scans and supply disks of appropriate size for cup formation to the cavity fabricator. These are scrap saving measures. The fabrication company would be responsible for ordering and accepting the $\mathrm{Nb}$ material.

It is clear that an area where $R \& D$ might clearly lead to cost reduction is in the production of the $\mathrm{Nb}$ material. Wah Chang is the major supplier. Working with this US company to reduce costs could be a valuable direction for US TESLA collaboration.

\section{Electron Beam (e-beam) Welding}

Because of the present large fractional expense in welding, the industrial study focused on this procedure and recommended using an e-beam welder with 3 vacuum chambers and vacuum load locks to cut down the over all pump down and welding time. This production line approach of welding in the center chamber, while mounting pieces and pumping down for the next welding cycle in the first chamber, and letting welded assemblies of the previous cycle cool down and removing them in the third chamber results in an estimates improvement of reducing welding time for a cavity from $35 \mathrm{hr}$ to 5 hr. This reflects the present status that it takes 1-2 minutes to do a weld but $1 / 2 \mathrm{hr}$ to pump down, thus putting a premium on matching the pump down time with the number of welds that can be preformed at one time. 


\section{$\underline{\text { Cup fabrication and machining }}$}

Machining of the cups is a major fraction of the present fabrication. The fabrication industrial study did not investigate ways to improve this area. The study cost was based on estimates obtained from machine shops used to machining these sorts of pieces. However we note that machining $\mathrm{Nb}$ is not straight-forward and is critical to the welding process. There could be an underestimate of the cost of this operation.

\section{Cavity flanges and interconnects}

Cavity flange interconnects rely on bolted flanges that use specially machined AlMg seals. These connections are at the cavity ends and at the input coupler. For 20,000 cavities, this is a tremendous number of bolted connections that must be extremely reliable. It would be good if some other engineering solution could be found. We note that the superstructure would reduce the number of flanges for a two-cavity unit from 6 to 3 .

\section{$\underline{\text { Cryomodule cold mass }}$}

The cold mass consists of the vacuum vessel, gas return pipe, cryo supports, $4{ }^{\circ} \mathrm{K}$ and $70{ }^{\circ} \mathrm{K}$ thermal shields, cryo pipes and superinsulation. The cold mass comes preassembled and ready for mounting of the cavity string. The most expensive part is the vacuum vessel followed by the gas return header.

The TTF cryomodule cold mass is on its $3^{\text {rd }}$ design iteration. The cost for first cold mass was about 3 times the present TTF cost of $190 \mathrm{~K}$ Euros. It is expected in production that the present cost can be reduced by about a factor of two. Zanon, the producer of the present modules, carried out the industrial production estimate. The quantity needed for TESLA is not sufficiently large to invest in infrastructure necessary to implement efficient mass production methods.

The evolution of the cold mass design is noteworthy as it clearly indicates the benefits of a R\&D program where iterative prototype designs can be carried out. The first design was found to be very expensive to manufacture. There was difficulty in obtaining the necessary alignment tolerances in production and in assembly. In addition, the method of fabrication of the thermal shield was expensive. In iteration \# two, the shields were simplified by using welded "finger" connections to connect the upper and lower sections and aluminum cooling pipes were welded directly to the shields. By iteration \# three, the diameter of the vacuum vessel has been reduced to a standard pipe size (38" dia. $\mathrm{x} 3 / 8$ " thick). The gas return header, which is the key element in the support and alignment of the cavities, is assembled with its interface connections to the cavities and support posts in such a way that the critical connection surfaces can be machined on a long mill. It is not important to get the header pipe straight except that the ends of the header must be within exact tolerance $(1 / 2 \mathrm{~mm})$ in order that mating to the adjacent module can be performed without header distortion. Using the header ends as exact 
reference on the mill, one face, (that connects to the support posts) is machined accurately with respect to the ends. Then the header is turned over and the other face (that connects to the cavities) is machined. By this method, exact reference of the posts and cavities to the header ends is obtained. Additional simplifications include machining the coupler flanges after they are welded onto the vessel so as to take advantage of the accuracy of the mill. The present module has a design where the cavities stay fixed in longitudinal position when the module is cooled down and the cavity mount adjustments are now orthogonal in $\mathrm{x}$ and $\mathrm{y}$.

From the industrial study it is not clear whether the $17 \mathrm{~m}$ module length will be cost effective or not (estimated possible savings 5\%). This length of module would require mill beds, which are not conveniently accessible. Issues associated with over the road transport and drop hatch size must be addressed.

One of the areas which might deserve review is the procedure for installation of the superinsulation. It is felt at this time that manually assembled and fitting of insulation is most effective and that this is a critical operation if minimum thermal heat leak is to be obtained. There are many penetrations through the superinsulation that make effective thermal insulation much more difficult than for magnet cryostats. For production, some sort of manufacturing procedure, not based on human handwork, would need to be implemented in order to obtain reliable and uniform results.

$\underline{\text { Module assembly manpower (12 m module) }}$

Module assembly presently takes 4 people 21 days. It is expected that, by the next assembly, this can be reduced to 4 people for 15 days. For production, a reduction to $75 \%$ of this is assumed (or 45 man days).

No particular innovations to save manpower have yet been considered, so the assembly manpower estimate may be conservative. (We note that superinsulation blanket installation takes $10 \%$ of the total time.)

Cavity string assembly manpower( 8 cavities plus magnet, BPM, etc.)

Assembly of the cavities into a string to be ready for installation into the cold mass presently requires 3 people $\mathrm{x} 5$ days. For production it is assumed that $70 \%$ (10 man days) will be needed.

\section{b. Areas deserving further study}

There is a need for a complete flow chart from cavity production through complete cryomodule.

The cavity testing program should be evaluated to see if it is optimum. Should some part of the testing be performed at the assembly plant in order to avoid excess 
moving of the cavities? Are the cavities and modules capable of transport with out loosing alignment and field flatness?

The cryomodules will have cavities with a spread in operating gradient. What is the strategy to optimize this effect? How much spread can be tolerated within a module or RF unit without reducing gradient of the better cavities? Some modules will doubtless have unexpected weak cavities. When will it be better to just detune a weak cavity? It is unlikely that modules with one or two weak cavities will be removed from the tunnel unless their cryo or vacuum integrity is bad. How much loss in energy can be expected from low quality cavity performance?

An installation and module RF commissioning plan should be developed.

Beam vacuum failure analysis is started but a report should be prepared.

It would be useful to conduct a study of failure modes, risk analysis and tolerable failure rate. For example the AES company in the US did a similar study for a superconducting proton linac for APT commissioned by Los Alamos. The useful output of such a study is to determine where reliability needs to be increased, or spares and back-ups need to be put in.

It may be possible to automate the superinsulation installation.

As noted in the cryomodule section, further definition of alignment requirements should be worked out and understood by all.

\section{c. Opportunities for US collaboration}

We saw several opportunities for US collaboration in the further development program for TESLA. Superconducting RF facilities now exist at several laboratories in the US, at JLAB, Cornell, Fermilab, Argonne and Los Alamos. There is much interest in linac-based light sources that would benefit from a US capability for TESLA cavities and cryomodules. Several US companies have produced cryomodule components for CEBAF and CESR. The AES company has produced niobium cavities for and APT could be interested if the US wishes to develop SC cavity capability. Certainly, US companies hold a leading position in klystron technology and production of high purity niobium.

A critical area that needs collaborator laboratory participation in TESLA R\&D is the area of testing. Module systems testing is key to determining just how well improvements work, testing the operability of integrated systems, and long term reliability. At present, at TTF, there is a delay of years between when cavities are built and when they are finally operated in a module with beam. There is a real need for test facilities out side TTF in order to be able to carry out more tests more rapidly. 


\section{B. Main Linac RF System}

The TESLA RF System is based on conventional RF technology. There is very little technical risk and prototype systems have been operating in TTF for some years. The low level RF (LLRF) is quite complicated and needs careful development. The one tunnel linac layout leads to the necessity for high voltage pulse cables in the tunnel to connect between the modulators in service buildings and the klystrons located in the tunnel. This requirement for up to 200 cables in some sections of the tunnel is the most unattractive aspect of the RF system design. The low level RF system must be located in the tunnel as well.

\section{High Power RF - Klystrons and Modulators}

The RF system consists of 572 klystrons in the Main Linac. 560 are necessary for $500 \mathrm{GeV}$ operation and remaining 12 installed spares provide $2 \%$ redundancy. There will be 26 additional high power klystrons for the injectors. The modulators are located in surface buildings (approximately 4,450 square meters in area distributed over three floors) located 5 kilometers apart for the current accelerator design. The klystrons/pulse transformers are distributed in the tunnel, approximately 50 meters apart. The klystrons/pulse-transformer units are connected to the modulators through high voltage pulse cables. Typically there are about 100 modulator units per hall and cables in the tunnel must feed about 50 klystrons in each direction from the hall. Each modulator/klystron supplies power to 36 cavities (four $12 \mathrm{~m}$ modules or three $17 \mathrm{~m}$ modules).

\section{a. Klystron tubes}

Development of $10 \mathrm{MW}$ multi-beam (7-beam) klystrons (MBK) is part of the base plan. Three such klystrons TH1801 have been delivered to DESY from the French company Thales-Thomson. The multi-beam approach allows for more efficient klystron efficiency $(70 \%$ initial goal, $65 \%$ already achieved, with 67-68\% being a more achievable goal) with modulator/pulse transformer requirements of $117 \mathrm{KV}$ at 131 amperes. (Older single beam klystrons typically have $45 \%$ efficiency.) A prototype klystron operating at 3-4 megawatts in the TESLA Test Facility (TTF1) has been in operation for one year. This lower power is all that is required for the current TTF1 operations. A second unit was operated for two months before pulse transformer arcing stopped testing. Once the transformer was repaired, tests resumed on the klystron but other problems were found with this klystron. This tube has been returned to Thales for their evaluation. A third klystron is in test at Thales and has met acceptance tests. It will be installed and tested at DESY. The klystrons require 150-200 watts of drive from a 400-watt solid state $1.3 \mathrm{GHz}$ amplifier (SSB in Iserlohn, Germany).

TESLA currently plans to run a total of 5,000 hours per year (HEP and accelerator studies). Between runs, the klystrons would be placed in a stand-by mode at a reduced cathode temperature resulting in an average stand-by lifetime of 500,000 hours. For this operations scenario, it can be calculated that there will be a continuing need for 
approximately 76 new or possibly refurbished klystrons per year. This klystron replacement rate is based on an assumed average operating lifetime of 40,000 hours per tube, based on a reliability projection for these multi-beam tubes by Thales, which also proposes possible modifications to the existing MBK tube to attain the goal of an average lifetime of 60,000 hours per tube (50 tubes consumed per year). Thales believes that for the present design, the most likely lifetime failure would be cathode emission reduction. The 40,000 hr calculation also assumes no klystron turn-on casualty rate during shutdowns for tunnel access. Only continued operational experience with these new klystrons will tell if these operational plans, klystron lifetimes, and production/refurbishment rates are matched. But it is almost impossible to ascertain if lifetime will be sufficient prior to actual TESLA operation. If the lifetime is lower, then there is a resultant penalty in commissioning time, operating costs, and integrated luminosity (up-time). We believe that further studies of the klystron expected lifetime and failure modes with consideration of potential improvements will be of great benefit.

During initial TESLA construction, the peak klystron production rate is estimated to be 143 per year, with an average of 100 per year. Thales plans to maintain part of this klystron production capability to meet the replacement demands (half new, half refurbished tubes). TESLA can continue operations at the full $500 \mathrm{GeV}$ level with $\sim 2 \%$ of the RF systems off. This margin increases at lower energy operation where a larger number of klystrons can be turned down. This will also cover non-catastrophic failures of the cryomodules where cavities can be detuned and machine operations continued until convenient time for replacement. It is anticipated to have tunnel accesses for klystron replacement approximately every month or so, depending on the statistical nature of failures If the early klystron failure rate is higher during machine commissioning, which is likely, then the commissioning period will be longer and more costly.

Each modulator/klystron station is expected to consume 134 kilowatts from the mains. Modulator efficiency of $85 \%$ and total RF system efficiency of $55 \%$ at 9.7 megawatts output have been measured. The RF power required for operation of 36 cavities is $8.3 \mathrm{MW}$. There is $10 \%$ regulation reserve and $6 \%$ waveguide losses budgeted, leading to the 9.7 MW requirement. Total AC power (for RF systems) for a $500 \mathrm{GeV}$ center of mass collider is expected to be approximately 75 megawatts, plus an additional $6 \mathrm{MW}$ for XFEL operations. The other main power user is the cryogenic system at about $24 \mathrm{MW}$ (see section D.2. Cryogenics). For possible early operation at higher energy (up to $\sim 750 \mathrm{GeV}$ at $\sim 6 \times 10^{33}$ Luminosity) without increased power demand or additional RF and cryogenics, initial plans would be to lower the pulse repetition rate and pulse length.

The TTF1 functions with vertically mounted klystrons. The final TESLA design has all of the klystrons located in the accelerator tunnel, hence, requiring horizontal mounting. Both klystron and pulse transformer would be exchanged as a unit during klystron replacement. This change of mounting plane requires additional engineering to insure optimal klystron performance. There are also issues with the oil containment for the cathode of the klystrons. The first klystron capable of horizontal operation is expected to be delivered in 2003. 


\section{b. Modulators}

Work continues on modulator development. The baseline design will utilize the "bouncer circuit" concept developed at Fermilab. The cost of producing the large number of modulators required was estimated by a German company, PPT. PPT has extensive experience in building modulators for TTF, PITZ, SLS at PSI in Switzerland, and MAXLAB in Sweden, along with improving existing modulators for LINAC2 at DESY and for DELTA at Dortmund. The modulators are physically quite large, as they require capacitor banks capable of storing 70 kilojoules. This large capacity is necessary for good voltage regulation; the bouncer reduces capacitor energy storage to a minimum.

The charging HV power supplies proposed for TESLA are designed to allow a constant charge rate and steady AC power loading. The charging power supply uses a number of industrial pulse width modulated (PWM) power supplies. A DESY-developed self-learning regulation system maintains constant power draw on the AC mains with a distortion limited by law to $<0.5 \%$ at $5 \mathrm{~Hz}$.

The main HV switch utilizes series Insulated Gate Bipolar Transistors (IGBT) for TTF, which is specified to be upgraded to Integrated Gate Commutated Thyristors (IGCT) for the TDR. A one to twelve step up HV transformer with a pulse length of 1.4 msec (cost estimated by $\mathrm{ABB}$ ) is located in the tunnel at each klystron.

The current design for the TDR eliminated the backup switch from the Fermilab prototype. This backup switch, along with capacitor bank crowbar is designed to minimize energy deposition during a klystron spark in case of failure of main switch. It was eliminated under the assumption that ABB pulse transformer would absorb most of energy during a spark. Recent Fermilab computer simulations indicate that due to bouncer circuit, more energy is available (up to 100 Joules, compared to the klystron specification limit of $<20$ Joules) than assumed in TDR. It is recommended that consideration be given to risk vs. increasing the modulator cost by a very modest $(\sim 2 \%)$ amount for a backup switch. TESLA is preparing to include backup switches to the IGCT assemblies, including upgrading the new modulators at TTF.

Research on novel and improved modulator designs is continuing in an effort to reduce the size and cost of the modulators. (See Areas for further R\&D at the end of this section) A Superconducting Magnetic Energy Storage (SMES) type modulator is in the R\&D stages at Karlsruhe Research Center.

For the modulators, there is very little technical risk. The technology is well known and various modulators have been operating at TTF for years. The still open design issues have to do with cost optimization. The current TDR model would have a single company be responsible for constructing and testing modulators and delivering units ready for installation and commissioning by TESLA.

There are of course reliability issues. It will be very hard to prove that the modulator systems will indeed have the required reliability. Here again, as much life 
testing and systems testing as possible is at least one way to at find the more obvious problems before production.

\section{c. Pulse cables from modulator to transformer/klystron}

The modulator pulse at $\sim 10 \mathrm{KV}$ is delivered to the pulse transformers/klystrons in the tunnel via specially designed triaxial cables up to 2.8 kilometers in length. The cable is a custom (for the TESLA project) 25-ohm triaxial design and is currently produced by two manufacturers. These cables are similar to those for power distribution and the costs are well understood. The center conductor provides the main pulse with the outer conductor reserved for the bouncer pulse. Four such cables are required for each klystron. There is a question whether the cable impedance would have to be matched to the klystron load, other than a terminating RC at the pulse transformer. It is believed that a continuous 3-kilometer length can be fabricated, eliminating any need for splicing. Testing the performance of such a long cable is desirable well before beginning construction of the final linacs.

The cable installation logistics have been left up to the manufacturer/installer. The cables will be placed in trays below the personnel walkway in the tunnel. The cable is specially treated to be fire retardant (VPE safety cables). Details of "random accessibility", along with pre-installed "spares", need to be addressed. Also the details of cable replacement have not been addressed yet, including the logistics of the upgrade to the TESLA-800 configuration. Although TESLA remains confident, we found it difficult to assure ourselves that there was sufficient tunnel space allocated to the TESLA-800 klystron cable plant. The arrangement of components inside the tunnel has recently been optimized, allowing for installation of all the cables needed for $800 \mathrm{GeV}$ operations.

\section{RF Distribution, Low Level RF, and Operating Margin}

\section{a. RF Power Distribution}

The RF waveguide power distribution system consists of two symmetric arms from the pair of klystron windows per klystron. Each arm supplies 18 cavities and consists mostly of commercially available waveguide components. The phasing of each branch of the power delivery system will be controlled by three stub waveguide tuners. The current installation at TTF has manually tuned stub tuners that have been calibrated empirically with the beam. The tuning for two cryo modules takes about 8-16 hours. Automation of this procedure is necessary for the final linac. The first motorized tuner has been delivered from Beijing Vacuum Electronic Research Institute (BVERI) in China and has been tested in the Laboratory. It will be hot tested during the superstructure tests in the Summer of 2002.

No pressurized air, SF6, or other conditioning systems are intended in the waveguide system.. There is a high power 400-kilowatt circulator located at each cavitypower input coupler. The circulators are manufactured by Domen SPA Ferrite of St. 
Petersburg, Russia. The high power loads are also manufactured by the same company. Directional couplers are from Spinner in Munich, Germany.

The power distribution system is made from parts from all these different vendors. A single vendor to integrate all of the components into one assembly would greatly assist in the installation of the extensive distribution system and reduce costs. Such vendor has yet to be identified.

\section{b. Low Level RF (LLRF)}

Extensive development and prototyping of the Low Level RF system have been carried out. The system utilizes digital techniques extensively. As presently configured it uses 7 DSP (Digital Signal Processors) processors and is complex. The long pulse length (one millisecond) allows for sufficient processing time for sophisticated feed forward and feed back schemes. A functional system is currently in operation at TTF1. In the prototype that exists, a complete relay rack of hardware is necessary to support one klystron station. Much effort must be put into the hybridization and compacting of this hardware.

The timing and synchronization required for the TTF1 is substantially easier than for the actual Main Linacs. Very little effort has gone into the design of the local oscillator (LO) distribution system. The baseline LO system will use a $9 \mathrm{MHz}$ reference that is multiplied by 144 at each station to produce the required $1.3 \mathrm{GHz}$ signal reference. Experience with operating machines and the effort at the NLC project indicate that distribution of stable phase reference RF over the length of the linac is a significant effort. Tunnel temperature variations play a significant role in control of global phase stability. The TESLA approach will use the distribution system to hold the reference to +/- 20 degrees. Beam phase measurements are then needed to achieve the 1 degree tolerance. A third fiber optic system will be used to inter-calibrate and compare against cavity measurements. This is an area requiring continued development. (See Section F 2. vii also)

No special temperature control of the linac tunnels is envisioned. The expected tunnel temperature for the TESLA project is on the order of 35 to $40{ }^{\circ} \mathrm{C}$. Some $75-85$ megawatts of power will be dissipated in the tunnel; most is removed by a high temperature low conductivity water (LCW) system. It is planned that all electronics will be located within temperature controlled (HVAC) containers. The tunnel temperature is expected to vary by up to $\pm 3{ }^{\circ} \mathrm{C}$. This temperature variation is consistent with that estimated by the RF people. While this range does not appear to present a problem with the high power RF distribution, it will have an effect on the distribution of the $1.3 \mathrm{GHz}$ LO.

Many other details will also be controlled by the LLRF. A sophisticated automated system for tuning the linacs will need to be developed as part of the LLRF system. The piezo cavity tuners will be part of the feed forward loops. The LLRF while advanced still requires engineering on many fronts. The main interlock protection of the 
RF cavities, when powered, will be by sensing and tripping on any deviation from expected of forward or reflected power from the cavities. This will be one of the critical exception handling tools for machine protection and operation. This feature is included under the klystron or high power RF interlock system.

While a number of individuals have worked on the low level system over the years, there are only a couple of people assigned full time. This system is critically important to the success of the linac commissioning and operation. It must have a dedicated group that can focus on the development. The right people need to be found early so that the system can be properly engineered and tested long before any production is started. This is an excellent area for collaboration from experts in other laboratories.

\section{c. Operating Margin}

Based on initial discussion with the TESLA Collaboration, we have referred throughout this report to a $2 \%$ excess in linac modules and RF systems. The primary motivation for this margin is to have "hot" spare klystrons that can be turned on when klystrons fail. The idea is that down days for klystron replacement would take place every few weeks or so.

As subsequent work has proceeded on the emittance preservation, it has become advantageous to operate some of the low energy modules considerably off-crest $\left(-27^{\circ}\right)$ for BNS damping. The rest of the linac is phased at $+5^{\circ}$. When these phases are taken into account with the same cavity gradient, then the energy margin is only $0.7 \%$.

Energy margin will be needed not only for klystron failures, but for modulator and low level RF faults as well. In addition, there will be cavity trips during operation, which, until reset, will take stations off-line. All these effects will tend to reduce beam energy if not dealt with in some other way.

There are a number of ways to restore energy margin at the few $\%$ level:

1) At $500 \mathrm{GeV}$, where RF power, rather than operating gradient, will probably be the limiting factor, cavity gradients can be increased to restore nominal acceleration at the operating phase. This need not necessarily take more RF power, at least for small off-crest beam phases.

2) The beam current (i.e. luminosity), rather than energy, can be reduced by the few $\%$ needed to maintain nominal energy operation as the reserve is used.

3) If the piezo tuners were to be implemented for $500 \mathrm{GeV}$ operation, there would be a saving of RF power needed for regulation of about 10-12\% of the total power. This should be sufficient to recover necessary reserve.

4) By changing to an 8 cavity cryomodule configuration (from 12), a $12 \% \mathrm{RF}$ power reserve would be generated. However additional RF stations would be needed.

Even though there are a number of alternatives given here, it is clear that technical contingency is an area that will need further study and review. However, we also note 
that the main issues for any of the linear colliders are not at the few $\%$ level, but are more likely at a more basic level.

\section{Industrial Studies, Costs, and Commentary}

\section{a. Industrial studies}

Industrial studies have been carried out for production planning and project cost estimating. In some cases industrial studies were not preformed, but quotations were obtained.

\section{Klystrons - Thales-Thomson}

Thales-Thomson has been, for many years, the supplier of the conventional TH2104C klystron that has been used in TTF. They are the developer of the TH1801 MBK multi-beam klystron developed for the high efficiency requirement of TESLA.

Thales carried out an industrial study for production of the multi-beam klystron. The study included additional development, industrialization, investment, and production. The study incorporated their knowledge of present construction techniques and how they would proceed to modify and automate production in order to meet the production rate demand. They would make changes for higher volume and lower cost in a number of steps. In their study, they opted to have full production of over 5 years. Total production including end effects takes 7 years. Peak production would be $\sim 144$ klystrons/year. The plant capability would then exceed the expected replacement/rebuild rate during operation, and keep the plant operating at a good fraction of capacity.

\section{Modulator- PPT}

The PPT study covered questions of additional development, industrialization, and production strategy. The question of cooperation with partner companies specializing in certain modulator subunits and the question of assembly and testing of the complete modulators, in order to assure quality, was discussed. These subunits included pulse transformers and IGCT switches from ABB. PPT assumed a total production time of 6 years for the modulators, including startup time with a reduced production rate.

\section{Circulators and Loads- Domen SPA Ferrite}

Ferrite provided a quotation on TTF circulators and loads. Discussion took place with Ferrite personnel on possible savings on mass production. DESY applied scaling laws to obtain projected costs for mass production. 
Drivers - SSB - provided quotations on mass production of drivers

\section{Directional Couplers- Spinner}

Spinner provided quotations on TTF couplers and straight waveguides and bends. Discussion took place with Spinner personnel on possible savings on mass production. DESY applied scaling laws to obtain projected costs for mass production.

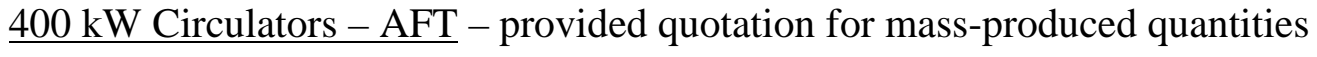

$\underline{\text { HV Power Supplies for Modulators }}$

Possible manufacturers are FUG and Beerwald who built HVPS for the new TTF modulators.

Pulse Cables-Nexans - provided quotation

\section{b. TTF RF Costs and Manpower}

The table below lists the major components and the approximate unit costs associated with the TTF installation. Here again, as in the Linac section, these estimates are our best determination, not detailed investigation. We give these costs as an of indication of the component cost breakdown and to show overall saving expected in mass production of the overall Main Linac RF System for TESLA quantities. 


\section{Approximate Present TTF RF System Costs}

(K Euro, assuming \$=Euro) $\frac{\text { Fermi Modulator }}{1996 \text { costs }} \quad \underline{\text { TTF Costs }} \quad \underline{\text { notes }}$

Modulator 209

IGBT switch 28

Pulse Transformer $\quad 110$

Ignitron 8

Interlocks and modulator controls $\quad 40$

Klystron auxiliary 6

Modulator total 401

(escalate $* 1.16$ for 2001 costs) $\rightarrow \quad 465$

adding escalated labor (see below) 337

less projected savings (see below $\quad-157$

total Fermilab modulator cost $\quad 645$

Klystron, solenoid, socket (TH2104C) assumes a $10 \mathrm{MW}$ tube costs same

Other

Pulse power cables

$\mathrm{RF}$ power distribution costs for $5 \mathrm{MW}$ circulators and associated

equipment has been subtracted because they

will not be used in TESLA

Interlocks (couplers $5.4 * 32$ )

Tunnel cable connections

LLRF

Driver

645 can drive $10 \mathrm{MW}$ tube

$1805 \mathrm{MW}-$ two 8 cavity modules $10 \mathrm{MW}$ - four 8 cavity

- $\quad$ not applicable at TTF

280 for four 8 cavity modules

TTF Total per system of four 8 cavity modules (32) $1467+?$ for cables, connections

TESLA Total per four 9 cavity modules (36) $\quad 1028 \quad$ (=587 ME/572 klystrons)

\section{Comments on Fermi Modulator Costs}

Fermi modulator parts costs are well documented. The numbers given above have been escalated by $\sim 1.16$ to bring to today's costs. The above costs do not include lab assembly and installation manpower. For the Fermi Modulator assembly manpower is estimated at $\$ 337 \mathrm{~K}$, escalated. (This is under the assumption that labor cost $=$ parts cost, counting only parts costs for items needing assembly, e.g. not the Pulse Transformer). German labor costs would be lower. 
Potential areas of savings, which have been identified for future prototype modulator development, are:

Savings Estimate - Labor \& Parts $(\mathrm{E}=\$)$

\begin{tabular}{lr}
\hline IGBT switch & $35 \mathrm{~K}$ \\
Hazy Self-healing Polypropylene Capacitors & $40 \mathrm{~K}$ \\
interlock/controls upgrade(2 windows) & $24 \mathrm{~K}$ \\
$5 \mathrm{~Hz}$ & $58 \mathrm{~K}$ \\
\hline total potential savings on modulator costs & $157 \mathrm{~K}$
\end{tabular}

With this saving one would expect for a Fermilab prototype modulator, if built now, a cost of: $465 \mathrm{~K}$ parts plus $337 \mathrm{~K}$ labor less 157 savings $=645 \mathrm{~K}$ total.

Simple "rule of thumb" mass production scaling with 5\% decrease for each doubling (95\% learning curve) would then result in production modulator cost of $\sim 400 \mathrm{~K}$.

\section{TESLA RF Manpower Estimate}

Greater than 1250 man-years of TESLA labor (18\% of the total) is expected throughout the construction period expressly for the RF systems. Over 200 FTE (fulltime equivalent) people are planned for the peak installation years. The TESLA RF Group manpower would perform management system control of RF components (EDMS), incoming inspection, preparation for installation, and quality assurance for the field installation by contractors. This would include rough adjustment of the components, e.g. for the phases of the waveguide distribution system and hardware checkout before beam is available. The fine adjustment is part of the commissioning and operation of the linac. This estimate does not include the manpower required for cavity testing or coupler conditioning. It is our understanding that the major body of these people will be associated with installation checkout, commissioning, and operation of the RF systems and cavities during the project period.

\section{$\underline{\text { RF Power Test Facility RF component test hall }}$}

A RF acceptance and test hall is planned. This facility will check and prepare components prior to tunnel installation. Infrastructure costs to set up this hall are included. An existing DESY hall will be used.

\section{Modulator Buildings}

Modulator buildings are planned in conjunction with cryo plant buildings. Space planned is approximately 4,450 square meters in area distributed over three floors (an increase from the TDR). 


\section{c. Tunnel configuration}

The one tunnel linac configuration has significant impact on the RF system configuration. This in turn will have potential impact on the RF system commissioning, reliability, and operability. The pros and cons of one vs. two tunnel configurations should be studied carefully to assess the potential impact (cost and technical) of both variants. This impact may be site dependent as well. The most unattractive features of the one tunnel layout from the RF point of view are the large number of pulse cables and the inaccessibility of the low level RF and interlocks. The inability to maintain tunnel RF systems while operating the linac will have impact on operability and availability. (Radiation damage to electronics and personnel safety are additional considerations.)

More compact modulator designs might be better suited to tunnel installation (in either 1 or 2 tunnel configuration), but certainly locating the modulators in groups of 100 in buildings appears to be a better maintenance feature than distributing them in the tunnel. This needs to be compared with the cable distribution. Modulators in the tunnel would allow for consideration of the SNS Los Alamos type of pulse width modulation pulse transformer /rectifier should it prove to be a reliable, space and cost saving option. Two tunnels would allow for complete assess to all the RF systems and repair/replacement during linac operation.

A careful study of all aspects of the 1-2 tunnel choice would be beneficial. Further $R \& D$ on the modulators for space savings (see below) would supply important information to such a study.

\section{d. Areas for further R\&D, Industrialization, and/or U.S. Collaboration Assistance in Modulators and RF Systems}

These could include the following items:

1: Currently there is only one manufacturer for modulators (PPT, located in Germany). An additional company capable of taking on the task has been identified in Boston, Massachusetts, but has not yet been contracted. Other US companies may be interested as well.

2: Thales is the only manufacturer of multi-beam klystrons. A contract has been placed with CPI of Palo Alto, California for delivery of a tube by early 2003. A Russian firm is in the process of providing a quote. It took Thales 5 years to develop a $10 \mathrm{MW}$ multi-beam klystron.

3: Reducing the size, complexity, and cost of the modulator controls and interlocks and integration of RF systems. Currently Fermilab is assembling an integrated interlock/control unit, which costs $\$ 18 \mathrm{~K}$ in parts (as compared to the $\$ 40 \mathrm{~K}$ for the original distributed system). This system could be used for future TTF modulators. 
4: High power RF component integration, e.g. mass production of integrated waveguide assemblies to reduce cost and installation complexity.

5: Simpler, cost optimized, and more compact modulator design. SNS Los Alamos is proposing the use of self-clearing polypropylene HAZY "traction" capacitors for energy storage; use of these capacitors would reduce the modulator size considerably. Although not currently an approach favored by TESLA, a Russian proposal for a mismatched pulse forming network (PFN) system should continue be evaluated and possibly a prototype developed.

6: A third source (in U.S.) of triaxial pulse cable.

7: Studies of commissioning, reliability, and operational aspects and scenarios. Experience is needed to discover the weak points, where improvements can be made.

8: Possible location of modulator in the tunnel, eliminating the need for the long pulse cables and allowing conventional AC power distribution. Consideration of SNS high frequency switch mode technique (if modulator could be located near klystron);

9: Development of LLRF components and LO distribution system, radiation hardness of components.

10: Continue implementation of a solid state back up switch for klystron protection. supply.

11: Development of a different solution for the constant charge rate HV charging 


\section{Tunnels and Buildings}

The description and definition of line elements of the TESLA WBS Dictionary for Conventional Facilities, based on the TESLA Budget Book (see Appendix), establishes a baseline for comparison, in terms of scope and content, with other design solutions for a Linear Collider project. The purpose of this study was to understand, in a general sense, what is contained in the TESLA Project so that accurate conclusions can be drawn. It is not intended to justify design approaches, unit costs or construction methods, or even the relative advantages or disadvantages of the national or international regulations and/or oversight organizations under which such a project may be constructed. Such in-depth analysis would require a much more concerted effort and at this stage of the various design efforts may be a bit premature. Nevertheless, fundamental differences do exist and they need to be identified in order to establish suitable levels of comparison. In any project (certainly one of this scale) when independent solutions are pursued, there is always a temptation to compare costs in an effort to rank the relative economy of one solution over another. While analyses of this nature are important, one must be very careful to "compare apples and apples". That is indeed the objective of these conclusions to follow.

The following analysis will be based on the Conventional Facilities information contained in the TESLA TDR and subsequent TESLA WBS Dictionary (see Appendix) and the information developed for the FY01 NLC design solution for the North/South, "less-deep" configuration at Fermilab including their current cost estimates. The TESLA XFEL was not considered at this stage of the comparative process. While unit cost comparison is not the objective of this report, percentages of costs that represent specific portions of the content of the total estimated costs are relevant. Therefore as a point of reference, the following total costs for Conventional Facilities will be used for the named projects. For the TESLA project, the cost of the elements described in the TESLA WBS Dictionary under TESLA WBS 1.1.3, "Tunnel and Buildings" (546 M Euro) as well as those contained in TESLA WBS 1.1.4, "Machine Infrastructure" (approximately $130 \mathrm{M}$ Euro for Water, AC Power, and Ventilation systems and for Damping Ring Infrastructure) total $676 \mathrm{M}$ Euro. The cost identified for the FY02 NLC North South configuration is $\$ 2,000 \mathrm{M}$ (rounded). For purposes of this analysis, the exchange rate between Euro dollars and United States dollars will be one to one, 1 Euro $=\$ 1$.

This analysis will identify four areas that represent fundamental differences in the way the total cost was developed for these two linear collider projects. In general they are:

1. Project Scope,

2. Oversight and Regulatory Requirements,

3. Relative Labor Rates, and

4. Construction Contingency, followed by a brief discussion of TESLA-specific topics of

5. Tunnel Siting, Stability, and Infrastructure. 
The conventional construction planning is being carried out by a DESY team, which includes a tunnel boring engineer (Lindemar Hänisch) from the Berlin subway, along with approximately 15-20 consulting engineers from Windels, Timm, \& Morgen, and from Amberg Ingenieurbüro and other companies.

\section{Project Scope}

Both projects, from the conventional facilities aspect, are intended to house a machine of relatively similar size and function. However the technology utilized for each of the different machines drives a very different configuration with respect to conventional facilities.

The TESLA design requires a $33 \mathrm{~km}$ long tunnel with only four "dog bone loops" for damping ring configuration and relatively simple additional tunnel construction for injection and positron by-pass of the interaction region. It is not necessary for the beam line to be laser straight and therefore the tunnel alignment follows the curvature of the earth minimizing the below surface depth in which it is to be constructed. It is a single tunnel design with regularly spaced shaft access for egress, material handling, piping for cryogenic and conventional mechanical cooling, HVAC support and connection to local electrical power supply. The tunnel construction is complicated by high water tables, which result in the requirement for a fully lined tunnel and specialized tunnel boring equipment. However the construction of the HERA tunnel and vehicle tunnels (Autobahn tunnel under the Elbe River) in the Hamburg area using the same construction method provides a good deal of experience in this construction technique. The TESLA design includes one interaction region and related beam dump configuration. Surface buildings include the 7 cryoplants (each 2,720 $\mathrm{m}^{2}$ ), 7 modulator/water plant halls (at the time of the TDR, each $\left.1,225 \mathrm{~m}^{2}\right)$, the access hall at Westerhorn $\left(400 \mathrm{~m}^{2}\right)$, and the module test building $\left(11,475 \mathrm{~m}^{2}\right)$, giving a total of $\sim 39,500 \mathrm{~m}^{2}$ or $\sim 425,000 \mathrm{ft}^{2}$. Since the TDR, the modulator/water plant buildings have increased to $4000 \mathrm{~m}^{2}$. (Note that there are inconsistencies in the sizes of these surface buildings between the TDR, the Budget Book, and the schematic table presented at Snowmass in July, 2001.) Additional office and support space that may be used for TESLA is considered to exist on the DESY site and is not included in the scope of this project. Therefore any associated cost to upgrade this existing space is also not included in this project. The acquisition of approximately 205 acres of land (which included the land required for the XFEL Complex) is included in the TESLA cost estimate. There is apparently no need, or provision, for the purchase of underground easements for tunneling. However, the TESLA project will have liability for any damage to private property, which may be an additional cost.

The referenced NLC design requires an overall tunnel length of $30 \mathrm{~km}$, which is laser straight and located in a rock layer averaging some 150 feet below grade. Furthermore, because of its depth and experience in exiting requirements from previous (conventional, non-accelerator) projects, a dual tunnel design has been incorporated. One tunnel will house the accelerator components and a parallel tunnel of equal size will house support equipment and satisfy egress requirements. In addition, there is a more 
complex configuration of injectors, pre-damping rings and damping rings centrally located in near surface enclosures and buildings in order to take advantage of the existing Fermilab site. Transfer beam lines and associated enclosures are also required to transport the beam to the ends of each Main Linac. This NLC design includes both a high energy and low energy interaction region and associated beam dumps and beam delivery enclosures. While the dual tunnel design minimizes the need for regularly spaced access shafts, some shafts are still required. Surface buildings specifically required for the injector complex total approximately $250,000 \mathrm{sq}$. ft. with additional new construction of anticipated support buildings totaling approximately $180,000 \mathrm{sq}$. ft. Further, the cost to upgrade and renovation of some 670,000 sq. ft. of existing Fermilab buildings anticipated for use on this project is also included in the total scope of the project. While a detailed functional analysis remains to be completed for the NLC project, and may reduce the building square footages noted above, these costs are contained in the existing estimate. There are approximately 16 acres of land beyond the Fermilab site needed for this project. Neither the cost of this land, nor that of underground easements is included in the current cost estimate.

It is clear that a detailed analysis of the information cited above is necessary before accurate conclusions can be made regarding cost comparisons between the "cost per meter" of the TESLA tunnel and the "cost per foot" the NLC tunnel. While that analysis is beyond the scope of this current effort one can identify some first order comparisons that give a general sense of differences in the unit costs utilized to generate portions of the current cost estimates. For the purpose of this report, a general comparison of the costs of tunnel construction, experimental halls and grade level buildings will be provided. Costs estimated for the TESLA TDR rely heavily on the experience gained during the construction of the HERA Project. It is anticipated that the ground conditions and construction techniques anticipated for the TESLA project will be very similar to those encountered during the construction of HERA.

With respect to tunnel construction, the unit cost for the Main Linac tunnel described in the attached appendix is $9,000 \mathrm{Euro} / \mathrm{m}$ or $\$ 2,750 / \mathrm{lin}$. $\mathrm{ft}$. (including the design and build costs). This is the cost used for a fully lined 5.2 meter finished diameter tunnel constructed in water bearing subsurface conditions. (Recently, the tunnel diameter has been changed to 5.5 meters.) The NLC unit cost for the Main Linac tunnel (average of 16 and $17.5 \mathrm{ft}$., 4.9 and 5.3 meter diameters) bored in dolomite rock with provision for $20 \%$ of its length to be lined due to potential water infiltration is $\$ 2,100 / \mathrm{lin}$. $\mathrm{ft}$. $(\$ 6900 / \mathrm{m})$. VLHC estimates for a tunnel to be bored in wet sandstone using similar technologies to the HERA/TESLA tunnel is $\$ 8,332 / \mathrm{m}$ (does not include invert floor). Costs for experimental halls can be compared in a similar manner although it should be noted that the TESLA experimental hall configuration is basically a rectangular box while the NLC configuration has both an underground component as well as a grade level access. This comparison will only take into consideration the below ground portions of the experimental halls. The TESLA cost estimate uses the cubic meter $\left(\mathrm{m}^{3}\right)$ as the standard unit of measure for this type of construction while the English unit convention is to measure building size in terms of square feet $\left(\mathrm{ft}^{2}\right)$. The cost factor for this standard unit of volume for the experimental hall is $600 \mathrm{Euro} / \mathrm{m}^{3}$. The TESLA experimental hall 
has a total volume of $61,500 \mathrm{~m}^{3}$; it measures, after translation to English units, $30,100 \mathrm{ft}^{2}$ in plan. This converts to a unit cost of $\$ 1,250 / \mathrm{ft}^{2}$. The NLC experimental hall $(73 \mathrm{ft}$. height) unit cost is $\$ 1,400 / \mathrm{ft}^{2}$, which translates to $\$ 677 / \mathrm{m}^{3}$ for drill and blast construction deep in dolomite. The grade level buildings utilize the same basic units of measure described above for the experimental halls. The TESLA grade level high-bay industrial buildings (10 meter height) use a cost factor of 75 Euro/ $\mathrm{m}^{3}$, not including offices and workshops, electrical, or HVAC. Translated into English units as above, this converts to a unit cost of $\$ 70 / \mathrm{ft}^{2}$. The NLC unit cost for new grade level high-bay industrial buildings is approximately $\$ 230 / \mathrm{ft}^{2}$, including customary basic utilities (including crane, but not special utilities for technical systems). There is a considerable difference here.

From the gross numbers indicated above, it is easy to see that a better understanding of the details behind these crude unit costs is essential to understanding the differences between them. It is true that all of these costs contain a large element of historical experience based on construction that has previously occurred at each of the respective laboratories. However they also reflect local labor rates, various levels of contractor input and different perspectives on what is considered to be a basic "barebones building". It should be further noted that there are many other aspects of these numbers that contribute to their similarity or lack thereof. The only certain thing that can be stated about the units costs indicated above is that none of them include any contingency. The TESLA Project has stated their reasons for not including contingency as a line item and the NLC adds contingency as a separate percentage mark-up in a different portion of the estimate. Before one questions why they are different, one must first ascertain what they represent and that will take a good deal more effort than has been expended to date. It is also important to note that a good deal of work also would be necessary before one might be able to calculate on the cost of building the TESLA machine in any location other than in Hamburg.

\section{Oversight and Regulatory Requirements}

This area of distinction address, in large measure, the differences between the way the content of the TELSA project scope was developed and the requirements for what we understand to be contained in the Department of Energy definition of Total Project Cost. The TESLA estimate is much closer to a DOE Total Estimated Cost (TEC) model. The scope of the TESLA cost estimate begins with the start of design and construction of conventional facilities. Because it is anticipated that most, if not all, of the conventional construction is intended to be under Design/Build contracts, this point in time signals the start of construction. This is an important point for two reasons. First, most of the A/E final design costs are included in the construction costs by virtue of the design/build process, and are thus included in the unit (per foot or per sq. ft.) costs. Second, a comparison of actual $\mathrm{A} / \mathrm{E}$ costs incurred for the project will be substantially different. In Germany, typically $13-15 \%$ of the cost of buildings up to $50 \mathrm{M} \mathrm{DM}$ is allocated for AECM costs. For HERA, the AECM costs totaled $7 \%$ of the conventional construction costs. The AECM support costs identified in the TESLA WBS for tunnel \& shafts and site \& halls represent approximately $2.7 \%$ of the construction cost which is primarily for the oversight of the construction process. Preliminary and Conceptual 
Design costs are also not included in the TESLA cost estimate. In addition, there is also a process to address safety and environmental concerns, legal aspects and public participation, which in German is called Planfeststellungsverfahren, loosely translated as the procedure to obtain legal approval to build. Since a research tunnel does not fit into any code, the particular safety considerations must be presented for approval to the Oberbergamt, the Head Bureau of Mining. It is hoped that the preliminary documentation will be completed and approval granted to begin public hearings by the end of 2002 . This process seems, in some respects, to correspond to our required Environmental Impact Statement. The cost for these activities is also not included in the TESLA cost estimate.

At this point a comment regarding the design/build process is appropriate. Simply put, the design/build process is a mechanism that combines the cost of final design and the cost to construct into a single contracted cost to the overall project. There are certain advantages to this way of doing business. If a design firm is working together with the company that will construct the project, there are economies to be gained in the level of detail required for both working drawings and specifications, since the drawings are not required to withstand the scrutiny of an open bid process. The responsibility to provide a building or tunnel of specific size and construction type in accordance with certain performance type criteria is provided by a single contract and source to the project as a whole. The result is savings for design costs as well as, to a certain extent, savings in construction costs. The savings in design costs are primarily due to the fact that less work actually needs to be performed by the design firm with regard to drawings and specifications. The major cost advantage to the contractor is the overhead of formal negotiations with an independently contracted design firm as well as the overall project management. In all cases, time is saved by the parallel nature of some of the design work and the elimination of another cycle of bidding and review between the completion of final design work and the start of construction operations. Schedule improvements in any project generally afford the opportunity for monetary savings in overhead and oversight costs.

The Design and Build contractor will include the AECM functions. TESLA would be responsible for performance specifications, conceptual design, technical aspects of bid packages, vendor liaison, quality assurance, and acceptance tests. This TESLA effort is included in the manpower estimate.

DOE Order 413 Section 3.2.1.1 does provide for design-build contracting of standard, not-unique components, however, with firmly defined needs and scope to prevent cost and schedule growth due to changing requirements after placement. Examples of such projects include road building, administration facilities, fire stations, etc. One would wonder if tunnels, cryo plants, and underground enclosures, as well as industrial buildings located on the surface, would qualify.

In terms of DOE Order 413.3 (see Section IV.F.), the time frame for when costs begin to be applied to the overall estimated project cost compares to the time at which Critical Decision 1 has been reached. However, in order to comply with the DOE 
definition of Total Project Cost (TPC), the NLC estimate must include Preliminary and Conceptual Design costs as well as final design costs. While the actual start of accounting for design costs for the TPC can be subject to discussion, the current NLC estimate includes an amount that totals $25 \%$ of the estimated construction cost for conventional facilities for all design efforts. This figure is intended to provide for all necessary preliminary design (5\%), conceptual design $(7.5 \%)$, and final design $(7.5 \%$ inhouse and 5\% Outside A/E). While these percentages may be adjusted in the future (e.g. the similar AECM activities for the even longer $235 \mathrm{~km}$ VLHC underground construction was preliminarily estimated at about $17 \%$ ), nevertheless, they represent a substantial difference for AECM between the TESLA and NLC cost estimates.

\section{Relative Labor Rates}

The topic of relative labor rates between Europe and the United States was discussed with colleagues at DESY. By any measure this is a "soft" topic when trying to estimate the costs for labor in a project of this size. However it was noted that in certain European professions and trades, annual salaries were thought to be only $\sim 65 \%$ of comparable professions and trades in the United States. This discrepancy is most likely attributable to the international exchange rate and not to other factors such as cost of living, union influences, etc. One thing is certain, this is an area that needs further investigation in order to fully understand differences in estimated costs. By way of example, a general rule of thumb in conventional construction is that costs often tend to be split equally between labor and materials. If one follows this model, and European labor rates are conservatively only $70 \%$ of those in the United States, the resulting differential estimated cost for construction in the United States could be $21 \%$ higher than a similar estimate developed based on the reality of European construction. This is a very simplistic example of a topic that needs further study, but certainly one that needs to be considered when trying to understand relative costs.

\section{Construction Contingency}

While this topic can certainly provoke widespread discussion and will continue to do so over time, its explanation in terms of this report is quite simple. There is not single line item in the TESLA WBS that addresses contingency. That is not to say that at some level, vendor estimates, HERA experience, and the overall development of applied unit costs do not contain some consideration of this topic. However from discussions with colleagues at DESY, it was apparent that there is at least a perceived fundamental difference in the way European contractors approach the bidding process. It was explained that while changed conditions and discrepancies do indeed come up in the course of construction, there seems to be a tendency to work out a way to complete the project for the agreed cost instead of immediately using the recourse of claims for additional money as is more the case in our litigious society. There appears to be a mechanism in place for sharing such risk between the customer and the contractor. Such risks can, of course, be minimized by investing in more extensive site geologic studies, up to $1 \%$ of the underground construction cost, beforehand, as appropriate. Be that as it may, however, there is still the simple fact that an average $30 \%$ contingency factor has 
been applied to the estimated total construction and $\mathrm{A} / \mathrm{E}$ costs for the NLC project. While one can indeed continue to discuss the relative merit of the European perspective or whether $30 \%$ contingency is too little or too much for an estimate in the United States, it is a fact that this multiplier is part of the current NLC estimate.

Below is a diagram that illustrates the relative impact of the four items that were discussed above. It should be noted that while numeric values are used for scale, it is more intended to be a visual representation rather than a definitive accounting exercise. It is intended to point out that there are substantial differences in the way total projects are defined between TESLA and the NLC process. Also the values indicated for the NLC are very broad based numbers and are subject further refinement. Escalation has not been considered in this comparison. In addition, as stated in the text above, there has been no attempt made to reconcile the exchange rates between the dollar and the euro. Numbers in parentheses are a reference to the items listed in the text above. 


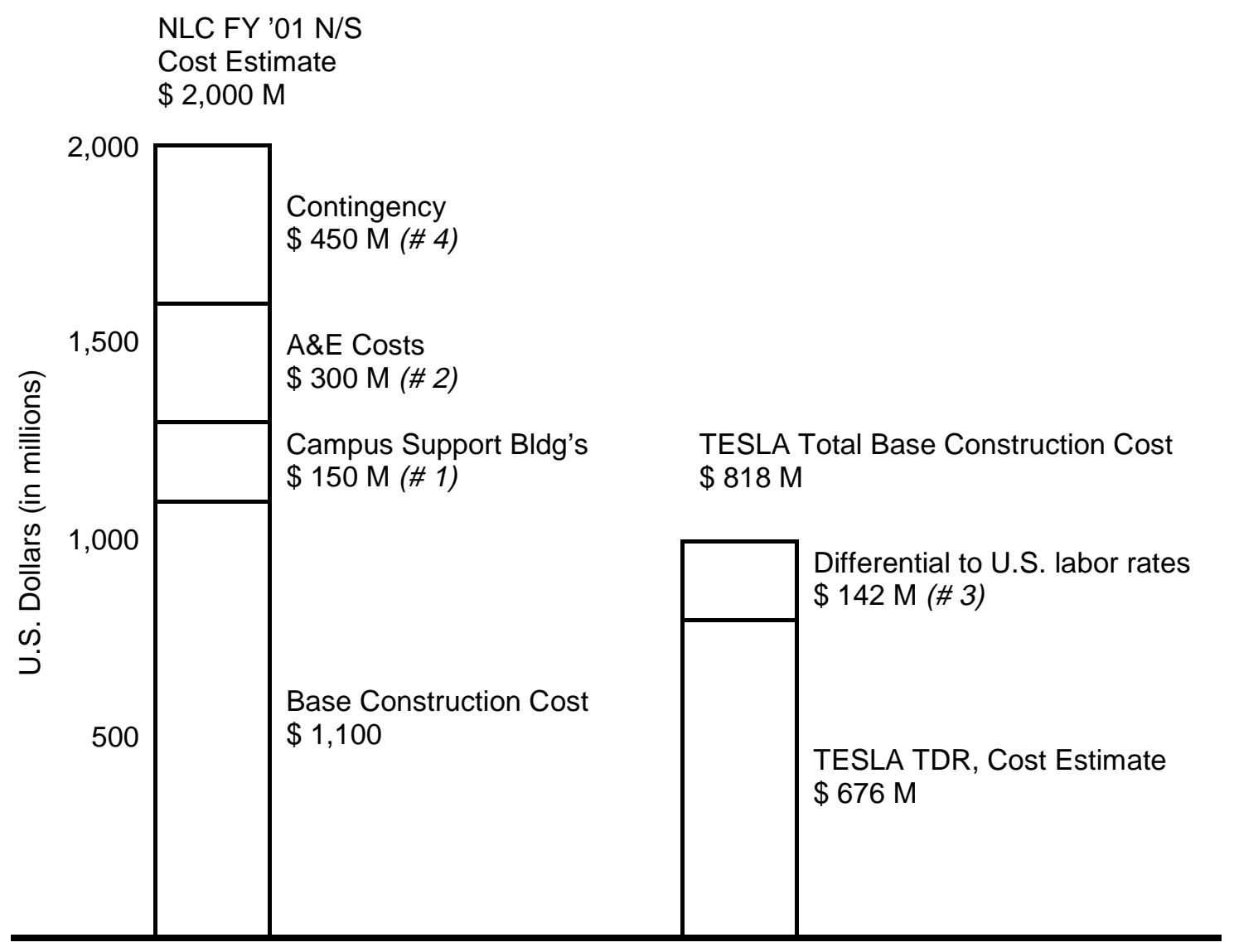

Conventional Facilities - Cost Comparison

At this stage of design maturity and cost estimate definition, the dollar values indicated are less important than the realization that fundamental differences in the content of the two estimates do exist. It only reinforces the necessity to understand these issues of content before one attempts to make any accurate comparisons. Further detailed analysis of construction methods, quantities and unit costs is needed, but as the graph above indicates, it will only serve to identify reasons for the approximate \$272 M difference in the normalized base construction costs. This is a good deal different than dividing the total numbers and reaching the singular conclusion that the NLC Conventional Facilities "cost" 2.6 times the TESLA Conventional Facilities. Both statements are correct in their own right, but highly dependent on what is included in the definition of "cost".

\section{Tunnel Siting, Stability, and Infrastructure}

The initial design of the TESLA project is for $500 \mathrm{GeV}$ center of mass collisions. Although the area chosen to site the project tangential to the DESY site clearly allows extension to the northwest to accommodate a longer tunnel for increased collision energies beyond $800 \mathrm{GeV}$, there is no plan to consider a longer footprint. Extension to the southeast is prohibited by the Elbe River, which is some 25 meters deep. Keeping the 
site for the proposed accelerator close to DESY allows taking advantage of the installed infrastructure on the DESY site.

Most of the data to support the proposed TESLA tunnel is based on and extrapolated from experience with the HERA tunnel at DESY. Over the years since construction (early 1980's), the HERA tunnel has shifted on the order of one millimeter. In recent years, new construction over the HERA tunnel has taken place by an outside developer. In this area, the relieved stresses in the ground strata have caused a 7millimeter shift in the tunnel. Since sections of the HERA tunnel are both in and above the water table, changes in the water table level have caused changes in the tunnel, as well as tunnel temperature variations, due to the different thermal characteristics between wet and dry soil. This might not be such a problem for the TESLA tunnel, which would be mostly under the water table, except for possibly for the area of the injector complex. For stability reasons, the tunneling technique used to build the HERA tunnel is clearly superior to cut and cover utilized at Fermilab where large tunnel motion (greater than 7 millimeters in the Main Injector/Recycler) has been observed over the last couple of years. Yet the above observations in the HERA locale may have some impact on TESLA machine alignment and support structures. We believe a review of all the alignment and tunnel stability issues would be worthwhile.

The expected tunnel temperature for the TESLA project is on the order of 35 to 40 degrees Celsius. Some 75-85 megawatts of power will be dissipated in the tunnel, most removed by a high temperature low conductivity water (LCW) system. This temperature variation is consistent with those estimated by the RF people. While this range does not appear to present a problem with the high power RF distribution, it will have an effect on the distribution of the $9 \mathrm{MHz}$ reference for the $1.3 \mathrm{GHz}$ Local Oscillator reference phase.

The amount of installed AC power at DESY is currently 100 MVA. The site actually consumes 50-60 MVA, which is closely monitored for peak, loads in the control room. The AC power consumption of TESLA 500 is estimated to be $155 \mathrm{MW}$ with approximately $1 / 2$ for RF systems and $1 / 6$ for Cryogenic Plants. These additional power requirements for the TESLA project do not present a problem for the available power on the local grid.

The civil construction also has impact on the size of the cryo modules. The original design has 15-meter diameter access shafts. The proposed size of the "superstructure" cryo modules is 17 meters. Lowering this longer device in the shafts would require some degree of tilt and a complete rework of the support structure for the cavities or a change in shaft size. At CERN, the length of the main dipoles was limited because of the existing shaft diameters. Magnet length will be set so they can be lowered in a level position. Weighing the costs of changing the TESLA cryomodule design over the diameter of the access shaft should be investigated. The present plan is to have shafts long enough to accommodate 17 meter long modules in a level position. 
The tunnel will have available three monorail movers (for tunnel components) per linac. Each has a safe lifting capacity of 12 tons. The current 15-meter cryo module meets this weight restriction. A 17-meter module would exceed the weight limit, requiring the use of two monorail units. The weight capacity of the monorail is dictated by the tunnel itself and is not clearly increased without large added expense. The weight of a superstructure and costs of increased installation time due to needing more than one monorail crane should be addressed. A new tunnel facility for TESLA Test Facility 2 (TTF2) has been constructed directly adjacent to the TTF1 facility. It is a full-scale 150meter long section of tunnel that will house the upgraded test facility. This tunnel has a monorail system installed. The completion of TTF2 will encounter most of the issues associated with systems installations before the construction of the final tunnels. Such a facility will allow for civil construction design changes before tunneling begins which is commendable and shows foresight. The planned diameter of the TESLA tunnel has now changed from $5.2 \mathrm{~m}$ to $5.5 \mathrm{~m}$.

Civil construction costs are based and prorated from experience with the HERA tunnel construction. It is estimated that the cost for the TESLA tunnels is 9000 Euros per meter. This sum includes ventilation and dryers to reduce humidity, but the other infrastructure costs such as plumbing for LCW, electrical installation, cable trays, and cryogenics. 


\section{Machine Infrastructure}

The Machine Infrastructure subsystem cost category (336 M Euros) includes the cryogenic plants, supply, and distribution ( $\sim 1 / 2$ of this cost element), other conventional utilities and systems, including Damping Ring infrastructure, which are typically included under the heading of conventional construction in the U.S. ( $~ 3 / 8$ of this cost element), and technical elements and operations related to component test systems $(\sim 1 / 8$ of this cost element). These test system technical elements are for the cryogenic and RF power test facilities. The cryo test facility is discussed in Section IV.A.1.

\section{Conventional Utilities and Systems}

These elements are described in more detail in section 1.1.4 of the Element Descriptions for TESLA Conventional Construction and Infrastructure in the Appendix. They include tunnel infrastructure, cable trays, power distribution (160 MW total), main power connections, cooling water $\left(30{ }^{\circ} \mathrm{C}\right.$ inlet $/ 70{ }^{\circ} \mathrm{C}$ outlet $)$ system, air conditioning and ventilation systems, and safety systems. An air drying system is required to reduce the dew point to below $12{ }^{\circ} \mathrm{C}$, the expected main tunnel wall temperature. The XFEL undulators tunnels need to be maintained at $22 \pm 0.3{ }^{\circ} \mathrm{C}$. 


\section{Cryogenics}

Cryogenics is divided between plants and distribution to the tunnel modules. Infrastructure such as power, water, and buildings is provided for in Section C (Tunnel and Buildings) or Section D.1. (Machine Infrastructure - Conventional Utilities and Systems).

The cryogenic system consists of 7 cryogenic plants, each typically capable of providing $4.2 \mathrm{~kW}$ refrigeration at $2{ }^{\circ} \mathrm{K}$, as well as $5{ }^{\circ} \mathrm{K}$ and $40{ }^{\circ} \mathrm{K}$ shield flows. A typical plant supplies 2 cryogenic units of modules, each $2.5 \mathrm{~km}$ long. The cryogenic units contain approximately 16 "strings" of 10 modules each. One module in each string is equipped with cryogenic valves and instrumentation (contained in the module costs). Each cryogenic unit contains 4 insulating vacuum barriers, dividing the insulating vacuum into 4 sections. Each plant has a distribution box that supplies the 2 cryounits and each cryogenic unit has a feed and end box. A plant has a cold box, warm and cold compressors, purification system, helium storage, and distributed control system capable of controlling both the plant and the integrated cryogenic module system in the tunnel.

During discussions, there were no identified potential R\&D topics. However, we believe that a process cycle simulation program would be of significant benefit. This program should be able to simulate the integrated plant and module system. It should be flexible The technical and cost risks were deemed to be either low or low/medium, and no budget contingencies were included in the TESLA estimate. However, there is a factor of 1.5 technical contingency in the sizing of the plants and the associated infrastructure.

The TESLA manpower effort requirements include: conceptual and system layout design; contract development; design review and acceptance; contract management; task management; and acceptance test management. Unless explicitly stated in the individual sections, there was no discussion on specific TESLA cryogenic personnel requirements nor sufficient breakdown to be useful for application to similar refrigeration systems.

Not explicitly called out is "cryogenic-infrastructure". This typically includes, if applicable: instrument quality compressed air, ODH systems, rigging services, etc. Much of this falls under the category of Miscellaneous.

\section{a. Cryogenic Plants and Supply}

The cost estimates for the cryogenic plants and supply systems were based on HERA and LHC (LHC Design Reports 317 and 391) experience, academic studies (under Dr. H. Quack of the Technical University of Dresden), industrial design and cost studies by Linde and L'Air Liquide, and budget quotations from Gardner Cryogenics.

During the study we noted that the intermediate piping costs to connect the various parts of the plant did not appear to be included. However compared to the LHC Tesla Engineering Study Review 
layout, the TESLA refrigerator components are situated closely together. The cost of interconnections between the various items plays a smaller role than with LHC. We have been assured that all such interconnection costs have been covered by the TESLA cost estimate.

Conventional infrastructure needed to support these installations should be included in Section C. (Tunnels and Buildings) or D.1. (Conventional Utilities and Systems above. This infrastructure includes, electrical power up to the equipment control panels, cooling water towers and systems for the Helium compressors, appropriate facilities (buildings with hatches and removable walls, building crane, sound proof walls, and tunnel shafts and caverns for sub-surface installation) with any required pipe ways, pits, foundations, and ventilation.

The Cryogenic Plant and Supply components include:

\section{i. Cold Boxes, Warm and Cold Compressors}

The cost basis was the LHC Design Report 317 augmented with Dr. H. Quack's cold compressor cost estimate, which was discussed with industry. The elements include the $4.5^{\circ} \mathrm{K}$ cold boxes, gas analysis system, motor starters, compressors, oil removal and oil pump skids, the $2^{\circ} \mathrm{K}$ cold boxes, cold compressors, cold absorbers, typical shipping charges, commissioning costs, and the required gantries and frameworks.

This element has a 50\% technical contingency since the cryogenics plant capacity is designed to be $50 \%$ larger than the anticipated load for TESLA-500. This element is deemed to have a low to medium technical risk and a medium cost risk which is driven by a potential lack of competition among industrial vendors.

\section{ii. External Purification System}

The cost basis was Dr. H. Quack's and industrial experience. This element included the external purifier and the purifier compressor.

\section{iii. Controls}

The cost basis was the experience of the air separation industry. This element included digital control system hardware and software specifically for the cryogenics plants and uninterruptible power supplies. This control system serves to control both the plants and the linac cryogenic module strings. (It does not include remote actuators and sensors located in the modules.) In addition, programming of the cryogenic controls and interfacing to the overall controls network is also required. (See findings.) The needed infrastructure includes the overall control system network and climate controlled control rooms.

\section{iv. Liquid Helium Storage}


The cost basis was a budgetary quotation from Gardner Cryogenics. This element includes $\mathrm{LN}_{2}$ shielded LHe dewars and LHe transfer tubes. A $2{ }^{\circ} \mathrm{K}$ load is integrated into the LHe storage dewars.

\section{v. Gaseous Helium Storage}

The cost basis is CERN experience. This element included gas storage tanks and associated piping.

\section{vi. Helium Inventory}

The cost basis for Helium procurement is DESY experience.

\section{vii. Miscellaneous}

The cost basis is DESY and Dr. H. Quack's experience. These elements include instrument air, $\mathrm{LN}_{2}$ tanks and associated distribution piping, and an "extra" $2 \%$ of the total cryogenics plants and supply cost estimate to cover missing elements.

\section{viii. Findings and Discussion}

The TESLA collaborators intend to have single contract for design, installation, and commissioning of the cryogenic plants. The contract will also include costs of connecting compressor skids, cold boxes and other elements of a turnkey cryogenic plant. However, the methodology used to arrive at the cost, LHC Design Report 317, excludes the following elements: piping contract to connect compressor and cold box skids, inventory control hardware, and $4.5^{\circ} \mathrm{K}$ and $2^{\circ} \mathrm{K}$ cold box test calorimeters. In the case of LHC, this added scope amounted to an additional $4 \%$ of the cryogenic plant cost predicted by the methodology used.

It is both CERN and DESY policy not to charge laboratory employees' time to a project. During our communication with colleagues from CERN/LHC we have discovered, that the part of the LHC cryogenic project cost overrun is related to the Industrial Support manpower cost. Over the years, reduction of work force at CERN has resulted in shortage of CERN personnel to execute completion of LHC cryogenics. This is particularly true in categories of technicians and designer/drafters. As a result, this workforce had to be contracted and charged directly to LHC project. This may not be a problem for TESLA project, however in case of LHC it has resulted in an unforeseen cost of $10 \mathrm{M}$ Euro $€$ or $\approx 4 \%$ of the cryogenic estimate.

No breakdown of cryogenic manpower requirements from DESY or other TESLA collaborating institutions for design, fabrication, installation, and commissioning was available or was included in the cost estimate. 
With international collaboration, there is a need and desire to award contacts to contributing countries. This can result in higher then anticipated shipping cost. In case of LHC, this contributed to the cryogenic cost overrun.

Partially due to an international collaboration and due to the limited number of cryogenic equipment vendors, there is an extra cost associated with maintaining competitive cryogenic equipment supply from industry by splitting contracts over multiple vendors. In case of LHC, this resulted in 5.5 M Euro extra cost.

Oxygen deficiency hazard $(\mathrm{ODH})$ safety systems are not planned and costs associated with ODH detection and abatement systems are not included. (See discussion 2e below.)

The TESLA cryogenic control costs, for both plants and cryomodules, were arrived at by using $10 \%$ of the cryogenic plant capital cost for the first plant, and $5 \%$ for subsequent plants. CERN LHC experience has shown the hardware component of controls to be considerably higher than $50 \%$, making software scaling for multiple plants less effective. item.)

Costs associated with the oil processing system are not included. (This is a small

Process cooling water towers are considering to be provided under the Machine Infrastructure (Conventional Facilities and Systems) element.

It is standard practice for European Laboratories like CERN and DESY not to use cost contingency for project budget estimating.

Cryogenic costs were not generated using a WBS (or equivalent) structure.

\section{b. Cryogenic Distribution}

\section{i. Distribution Boxes}

The cost basis was on a DESY experience-based equation based on the size, quantity, and costs of valves plus instrumentation, heat exchangers, vacuum barriers, etc. The sources of information are previous procurements of valve boxes, industrial valve prices, and detailed flow schematics with valve lists. This element includes valves, internal piping, external piping connections for integration with the refrigerator cold box, cold compressors, cryogenic units, and damping rings, low temperature counterflow heat exchangers, vacuum shell, and $80{ }^{\circ} \mathrm{K}$ thermal shield. $4{ }^{\circ} \mathrm{K}$ and $80{ }^{\circ} \mathrm{K}$ test calorimeters are incorporated in the distribution boxes.

\section{ii. $\quad$ Findings and Discussion}


Valve box and distribution box cost estimates by DESY for TESLA are based on three sources of information. One is previous experience in valve box procurement. From their latest procurements of valve boxes (HERA-FEL-valve-box, FEL-subcooler box, HERA-H1 and HERA-ZEUS luminosity up-grade valve boxes), DESY created a "rule-of-thumb" for the overall average costs of cryogenic valves of usual sizes inside a box. This rule-of-thumb includes all the tubing and other equipment. A second source of information is industrial valve prices. For the large valves in the $2^{\circ} \mathrm{K}$ return circuits (up to DN250), DESY obtained estimates from the WEKA company in Switzerland, one of the only suppliers of cryogenic valves of these sizes. The resulting valve cost estimates agree well with those used by CERN. Lastly, detailed flow schematics and valve lists were used in cost estimation. Figure 8.7.2 in the TESLA Technical Design Report shows only a simplified scheme for the distribution boxes. There will be different distribution boxes, which must also include the supply for the injector linacs and the damping rings. DESY made preliminary layouts of the distribution boxes including more or less complete flow schemes, and DESY made preliminary valve lists for these boxes. A design study has been started for one of the more complicated valve boxes.

Cost estimates for the distribution boxes came from Equation 1, which includes instrumentation. Note that based on the DESY valve box procurement experience previously mentioned, the "valve" cost is actually a cost of all the tubing and other equipment as well as the cost of the valve itself. Thus, total procurement cost is being scaled from the number and sizes of valves. This is the cost to buy the box, and presumably does not include installation costs.

cost of a valve box $=\mathrm{N} 1 * \mathrm{AC} 1+\mathrm{N} 2 * \mathrm{AC} 2+$ SofINSTR + Specials

where: N1 - number of "standard" valves < DN 250

$\mathrm{AC} 1$ - average overall costs of one valve of type $\mathrm{N} 1$

$\mathrm{N} 2$ - number of large valves DN250

AC2 -average overall costs of one valve of type N2

SofINSTR - sum of instrumentation (complete channels for temperature, pressure and flow sensors)

Specials - estimates for heat exchangers, vacuum barriers, etc.

(The TESLA distribution boxes will contain the low temperature heat exchangers and transfer connections to the cold compressor sets, not shown in Figure 8.7.2.)

For the special string end module cost estimates (feed boxes and end boxes), DESY added valve costs $(\mathrm{N} 1 * \mathrm{AC} 1)$ plus instrumentation costs to the module costs. (These, and the vacuum breaks, are included in the cryogenic distribution costs.)

For the injector linac feed boxes concept, DESY copied the Fermilab-TTF-feed box since the injectors will look similar to the TTF linac.

DESY did not obtain an industrial supplier's estimate for the distribution system. During their latest procurement procedures, DESY found quite large price differences Tesla Engineering Study Review 
between the different suppliers. Large price differences are not uncommon in cryogenic box procurements. Six quotations in 1999 for one magnet test feed box for the Fermilab Magnet Test Facility ranged from $\$ 73,085$ to $\$ 435,000$ with the median at about $\$ 150,000$. The Fermilab estimate, based on a scaling process similar to the one used by DESY, had been $\$ 230,000$. Only the high bid was from a European company. All bids were from experienced manufacturers of cryogenic equipment, including valve boxes and dewars.

Given the large range of quotes in the final procurement process, following a "rule of thumb" to scale prices as DESY has is a perfectly adequate way of making cost estimates.

The costs are for the cryogenic valves, actuators, instrumentation, and controls in the cryogenic string connection boxes depicted in TESLA TDR Figure 8.7.3 are included in the costs for the Cryomodules, Section III.A.2.

\section{c. Cryogenic Instrumentation}

There is little information available in the TESLA TDR detailing the cryogenic instrumentation and its costs for the project. The basic plan divides this instrumentation into two groups, (1) instrumentation associated with the cryogenic modules and (2) instrumentation associated with the cryogenic plants. The cryogenic instrumentation of the linac cryogenic modules was estimated to be 10.2 M Euro. These costs are included with the Main Linac Module estimates. Although a detailed list of instrumentation for the modules exists, it was not reviewed in sufficient detail to understand if this estimate is reasonable and all-inclusive. The plants' cryogenic instrumentation is included in the base costing of the plants.

\section{d. Cryogenic Operations}

Optimization of operating and capital costs was considered with the additional constraint of turndown capability. (Turndown means running a refrigerator at lowered capacity without a large sacrifice in efficiency. This would occur during times when the $\mathrm{RF}$ is off, or if the actual static heat load would be lower than design estimates.) The existence of a sub-atmospheric warm helium compressor allows for a wide range of system turndown. The proposed cycle has been successfully used in several existing low temperature cryogenic systems requiring turndown. The plants are foreseen to be fully automated to operate unmanned with a high availability.

\section{e. Oxygen Deficiency Hazard in the TESLA Tunnel}

There are presently no plans to include oxygen level detection equipment in the TESLA tunnel, thus no cost estimate was included in the TDR. The basis for this decision was experience gained with the operation of HERA, which also does not have oxygen detectors in their tunnel. Since both tunnels are of similar design and have the same inside diameters, this assumption appears to be reasonable. Additionally, HERA 
personnel conducted two 500 liter liquid helium dewar spill tests in their tunnel which demonstrated that an oxygen deficiency hazard (ODH) condition was relatively short lived and confined to a very localized area in the immediate vicinity of the spill location. Therefore, personnel in the tunnel near the release point would have sufficient time, given the visual and audible indications accompanying such an event, that a safe evacuation could be accomplished. Liquid Nitrogen will not be used in the TESLA tunnel.

We would recommend that the consequences of a long duration release of warm, gaseous helium from the system be analyzed before a final decision regarding oxygen detectors for the TESLA tunnel is made. The accumulation of helium gas in the upper portion of the tunnel due to an extended GHe release might result in an ODH condition if insufficient tunnel air exchanges occur. Since the equipment configuration in the tunnel results in the personnel walkway being located almost at the mid-plane of the tunnel, the person's head and shoulders will be in the upper third of the tunnel volume and could expose them to low oxygen concentrations should the ventilation rate be inadequate. We also recommend that TESLA personnel consider stratification in the helium-air mixture produced during a cold gas release scenario. A recent study conducted and detailed in LHC Project Report 508 might prove useful for this assessment.

\section{f. Alternative Options for a deep site}

The proposed cryogenic design could be used for a deep site installation. The cold box would have to be split into above and below ground boxes at about $20{ }^{\circ} \mathrm{K}$ level, similar to the LEP installation. The below ground cold box will contain low temperature heat exchangers, cold compressors and expanders. Additional requirements for deep site would include extra length of a helium transfer line and headers, tunnel level caverns for the equipment, power and cooling water utilities.

\section{g. Conclusion}

LHC design report 317 is used to estimate cost of a reference refrigeration system elements consisting of cold box, compressor skids, oil removal skids and oil pump skids based on $4.5 \mathrm{~K}$ equivalent load. This methodology is consistent with one described in LHC Design Report 391 for $1.8 \mathrm{~K}$ system, when heat load at given temperature level is properly converted to $4.5 \mathrm{~K}$. DESY experience is used to estimate cost the of the TESLA cryogenic distribution system. HERA and LHC experience, industrial and academic studies were considered in the cryogenic technical design and costing.

The level of thought and detail presented in the TESLA TDR by the TESLA collaborators is very impressive. The report addresses the major issues associated with a cryogenic system related to building a superconducting linear collider. It is evident that years of discussion, collaboration and required $\mathrm{R} \& \mathrm{D}$, have been compiled into a workable design. We would like to commend the authors for considering the operational impacts when designing the system. The extensive use of LHC and industrial experience 
reduces technical risks and optimizes the cost effectiveness of the TESLA cryogenic system. 


\section{E. XFEL Increments}

\section{a. Introduction}

This section reviewed the incremental costs associated with the x-ray FEL (XFEL) facility to be built along with the $500 \mathrm{GeV}$ Linear Collider construction described in the TESLA Technical Design Report and a subsequent presentation to the German Science Council.

The XFEL concept described in the TDR shares a part of the collider electron LINAC up to the $50 \mathrm{GeV}$ station in an alternating pulse mode. The estimated incremental cost associated with this design for the accelerator systems is 242 M Euros. In addition, there will be a need of 662 full-time equivalent man-years from DESY and collaborating institutions for these XFEL increments.

Since the publishing of the TDR, a subsequent proposal investigated an independent linac for the FEL, which would be built along with the TESLA linac and would share utilities between two linacs. This scheme would de-couple the operating parameters of the collider and XFEL programs. The estimated incremental cost for the independent linac scheme for accelerator system would rise from $242 \mathrm{M}$ Euros to $464 \mathrm{M}$ Euros. This proposal has been adapted and is now part of the baseline design and is being incorporated into the Planungfeststellungverfahren.

This study has not addressed the additional FEL experimental equipment costs estimated at $290 \mathrm{M}$ Euros for 10 beam lines, undulators, and 20 experimental stations. However based on the APS/ANL and ORNL/SNS experience, the estimate seems to be adequate for the initial complement of experiments.

\section{b. Description and Scope of the X-FEL}

The TESLA project proposes to build a coherent hard x-ray source using SASE (self amplified spontaneous emission) principle with a source peak brilliance better than $10^{34}$ photons/sec $\mathrm{mm}^{2} \mathrm{mr}^{2}$ with $0.1 \%$ energy bandwidth as an integral part of the collider facility. This peak brilliance is 10 orders of magnitude higher that existing $3^{\text {rd }}$ generation $\mathrm{x}$-ray sources. It is anticipated that a source of this brilliance with both spatial and temporal coherencies would open new realm of research in atomic and molecular, biological, chemical, condensed matters, and materials sciences.

We note the very significant result from TTF FEL operation, namely the successful laseing in the vacuum ultra violet (VUV) region at wavelength of $80-120 \mathrm{~nm}$. A power gain of $10^{7}$ has been obtained. At saturation, GW peak power is produced with a high degree of transverse coherence. (V. Ayvazyan, et al., Phys. Rev. Lett. $\underline{88}, 104802$ (2002)). The pulse length can be adjusted between $30-100 \mathrm{fs}$. Peak brilliance is about 8 orders of magnitude higher than that of $3^{\text {rd }}$ generation light sources. These results at TTF are a critical step in understanding and implementation that is needed for the XFEL proposal. 
Two key components of an XFEL source are: 1) a high-energy linac with high peak-current and small emittance beam, and 2) a long undulator. To make such a source a user facility for multiple beam lines to accommodate many scientific programs simultaneously, the linac must have high average beam current as well as high peak current. High average current has its origin in large number of bunches in a macro-pulse, and these bunches can split into several bunch trains containing smaller number of bunches. Such a high-energy high current linac is available using TESLA technology.

It is generally agreed that a linac of 15 to $50 \mathrm{GeV}$ in energy is required for such an XFEL facility. Although operational machine characteristics may not be same for XFEL use and for collider use, e.g. the XFEL use may not require such a high gradient compared to the collider use, the technology is same. Therefore, there are several advantages as to construct and operate both machines together. There would be substantial construction cost savings due to integrated design effort, quantity purchasing, and shared infrastructure to name a few examples. There would be also savings of operating costs due to the shared infrastructure, expertise, and operating crews.

The original proposal described in the TESLA Technical Design Report (TDR) was to use sections through the $50 \mathrm{GeV}$ station of the electron linac for both the collider and XFEL modes by an appropriate arrangement of pulse structures. For example, this portion of linac operates at $5 \mathrm{~Hz}$ for the collider mode alternating pulse-to-pulse with 5 $\mathrm{Hz}$ for the XFEL mode, resulting in $10 \mathrm{~Hz}$ effective operation for those sections of the linac. The plan was to be able to extract the linac beam in two different places for the FEL uses. One port would allow extraction in the 13-27 GeV range and a second in the 20-50 GeV range. In this coupled-operations scheme, one would expect much interference in scheduling, operations, and maintenance between the collider and FEL modes.

An additional complication in the scheme is that the FEL injector system is located at DESY site as the collider injector, and the FEL experimental area is located some $16 \mathrm{~km}$ away in the same campus as the collider detectors. This implies the FEL electron beam must be transported an extra $10 \mathrm{~km}$. Since the publication of the TDR, the TESLA collaboration has preformed a study for having an independent linac for the FEL program.

The alternate option (now the preferred option) is to build an independent and dedicated linac of an energy more optimized for the XFEL and located in a separate enclosure. This linac would share utilities and other infrastructure with the collider linac. The FEL injector would be moved away from the DESY site, and located some $4 \mathrm{~km}$ from the undulator area. DESY documented this alternate option together with the additional cost requirements.

The original TDR estimate of the incremental costs for FEL accelerator and associated infrastructure was $241 \mathrm{M}$ Euros. This included the FEL injector, additional RF power sources, tunnel, electron beam transfer lines, and utilities. The FEL experimental 
equipment cost estimate was 290 M Euros, which covered undulators, beam lines, and experimental stations of 5 coherent x-ray beam lines with 3 experimental stations per beam line and 5 other beam lines with one experimental station per beam line.

The updated DESY estimate of the FEL accelerator part incremental cost with a separate $50 \mathrm{GeV}$ linac is $464 \mathrm{M}$ Euros. This number should be compared with $241 \mathrm{M}$ Euros for the original scheme.

In this study, we followed the steps taken by DESY and TESLA collaborators in estimating the costs. Since the $50 \mathrm{GeV}$ accelerator is only $10 \%$ of the collider linac, the DESY and TESLA collaborators scaled from the Main Linac and its enclosures and support structure for most of the FEL cost estimates. Those elements unique to the FEL facility were estimated by DESY and TESLA collaborators. This was the methodology used for the TDR estimates.

For the new estimate for the independent linac scheme, an add-and-subtract method was used to obtain the incremental cost of the separate-but-simultaneous construction of the integrated collider-FEL facility.

\section{c. Discussion on the TDR Estimate for FEL Increments}

The initial incremental cost for the FEL accelerator system addition described in the TDR consists of:

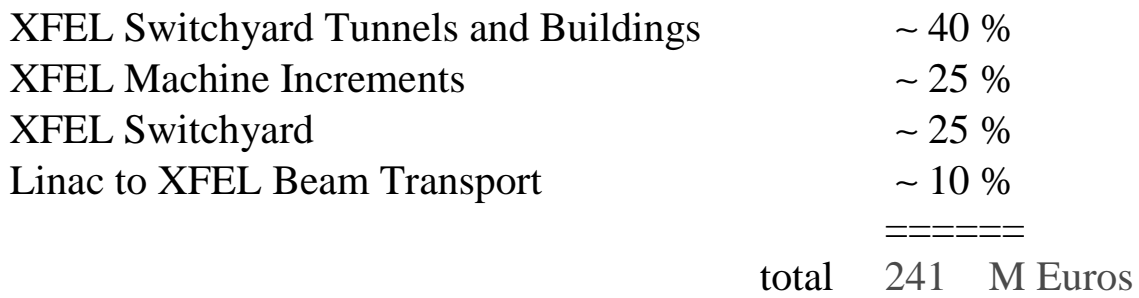

XFEL Tunnels and Buildings - This sum of funds covers the costs of switchyard tunnels, switchyard infrastructure, experimental hall and beam dump halls.

The total length of the switchyard tunnel is $8,000 \mathrm{~m}$. DESY estimates 7,500 Euros/m for these tunnels based on previous tunneling experience. Also included is the conventional infrastructure associated with the tunnels. These appear to be reasonable estimates.

The XFEL Experimental Hall has a dimension of $15 * 50 * 200 \mathrm{~m}^{3}$. DESY uses a unit cost of 150 Euros $/ \mathrm{m}^{3}$, a reasonable unit cost for a high bay building of this size. One might question whether a height of $15 \mathrm{~m}$ is necessary.

There are 4 underground beam dump buildings of $20 * 15 * 20 \mathrm{~m}^{3}$ with a unit cost estimate of 500 Euros $/ \mathrm{m}^{3}$. It would require detailed designs of the beam dump structures 
in order to assess the cost estimate. The experience at the SNS shows that high power beam dumps can be very complicated and expensive.

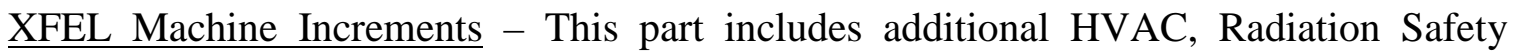
System and Interlocks, XFEL Gun and Injector, and additional water plant and distribution. Although we have no detailed design information, the estimated cost of each item appears to be reasonable.

XFEL Beam Switchyard - This section includes beam dump components, dipole magnets at 30,678 Euros each, and quadrupole magnets at 10,226 Euros each. These magnet costs are reasonable estimates.

Linac to XFEL Beam Transport - not discussed.

\section{d. Discussion on the Independent XFEL Linac Cost Estimate}

As noted earlier, this part of estimate used an add-and-subtract method. The subtraction amounted to $37 \mathrm{M}$ Euros from $241 \mathrm{M}$ Euros described in the previous section. This $37 \mathrm{M}$ Euros came from the fact that no additional RF, cryogenic, and beam line to XFEL from the linac described in the previous section are needed for the independent LINAC scheme. However, the cost estimate for the stand-alone XFEL LINAC assumed that it was constructed along with the Main Linac to take advantage of the large industrial procurement scale and the technical expertise of TESLA.

The added items are $10 \%$ of linac modules for the stand-alone linac, $10 \%$ of main RF system for the RF system of the new linac, $10 \%$ of Main Linac machine infrastructure for the new linac, etc.

Total incremental cost for the stand-alone XFEL Linac built at the same or contiguous time scale as that of the collider linac then rises to 464 M Euros.

\section{e. Manpower for XFEL Increments}

The total laboratory manpower needed for TESLA was noted in the TDR as 6933 FTE years. Of these, 662 FTE years were earmarked for the XFEL program. When one takes into account the fact that the most of hardware would be designed and constructed along with the Main Linac systems, an average of 83 FTE per year (peaking at 113 FTE per year) for the FEL design/construction is reasonable for the ED\&I (engineering, design and inspection) of the FEL portion of the work, not including the overall project management. It should be noted that the majority of accelerator ED\&I is to be done by the collider construction. 


\section{f. Assessment and Conclusion}

The incremental cost estimate (beyond TESLA LC estimate) for the stand-alone linac scheme is now $464 \mathrm{M}$ Euros, not including ED\&I and project management costs. This study reviewed these incremental estimates for the FEL accelerator, finding that:

1. The majority of the XFEL subsystem cost estimate is scaled from the Main Linac and infrastructure.

2. The cost estimates of those items unique to the XFEL program are quite reasonable.

3. The estimates of manpower effort requirement seem reasonable.

With respect to "independent LINAC" scheme for the XFEL, this study believes that there is a possibility of further optimization to reduce costs. The LINAC energy could be sufficient at 17 or $25 \mathrm{GeV}$, rather that at $50 \mathrm{GeV}$. Is a maximum energy of 50 $\mathrm{GeV}$ really required?

In January 2002, TESLA submitted a paper to the German Science Council discussing a staged installation for the XFEL, starting with and energy of $20 \mathrm{GeV}$, but leaving sufficient room to raise the energy at a later stage to $50 \mathrm{GeV}$, if required.

For the stand-alone XFEL scheme, it may be very useful to optimize not only the machine energy but also its buildings and tunnels. For example, what are the optimum depths for the XFEL LINAC tunnel, switchyard, beam dumps, and experimental hall?

The optimization of the tunnels and buildings is under study, as is the desirability of an initial phase with reduced energy and fewer beamlines. 


\section{F. Project Coordination, Overview, Integration, Logistics, and Management}

(Note: in the Appendices, there is a Glossary of U.S. and DOE Terms and Acronyms in common words, which may be helpful in reading this section.)

\section{Introduction}

This chapter includes several items that span the entire TESLA project. Specifically the proposed overall Project and Cost model will be discussed relative to procedures followed in other large modern projects. This section will first discuss the process used in the TESLA cost estimate, then laboratory personnel, testing and installation. The last sections will discuss the project model and project management.

TESLA has been conceived as an international project from its beginning. The $R \& D$ is being carried out by a multinational collaboration. It is fully realized that an international project management organization and procedures will need to be created and worked out that incorporate costing and management procedures of the various collaborating institutions and that provide international project oversight. Clearly developing such governance will take some time and needs the active participation of the major collaborating institutions. It will doubtless reopen and modify many of the project and cost models used in preparation of the TDR and project costs. This is understood by TESLA management. Be that as it may, some model had to be chosen in order to prepare the TDR. That model was the one most familiar to the group, i.e. the German model close to that used for HERA.

The DESY project model is different from the standard U.S. procedures. It has been used successfully in large projects like HERA. Main differences are associated with contingency and laboratory personnel. Other European projects follow a mixture of procedures. The scope of TESLA is of a scale of magnitude larger than HERA and that scale will need to be planned for.

Project management and critical milestones are also different. We will try to compare the status and steps of TESLA with DOE type procedures. The German procedures to start a project appear to be more time efficient and more pragmatic leading more quickly to the possibility of a project decision.

Use of project management and systems engineering tools are as yet mostly undeveloped at DESY. Use of these tools at some level will surely benefit such a large project as TESLA. It is getting them in place soon enough to do the most good, and getting the optimum level of implementation and use, which will be challenging. This is an area for possible collaboration. 


\section{International Engagement and TESLA Project Methodology}

As TESLA proceeds toward becoming a global laboratory, it may be necessary to accommodate and assimilate some practices that may be outside standard procedures of a German project. These include not only management practices, but also technical design choices. Where possible, TESLA should be careful not to foreclose design options at too early a stage without partner participation. If the final project collaboration is to be successful, it will be important to have broad participation in selection or review of design options. DESY should not get too far in front in making these decisions unilaterally. But this of course, is in conflict with (argues against) the necessity and natural inclination to get on with the decision process. TESLA cannot wait for project collaborators in order to move ahead, but it must be willing to revisit decisions when the project collaboration has been formed. Early involvement of collaborators is very important. There is a lot to do and there are technical choices that need working out.

When the collaboration for the project has come together and responsibilities are being distributed, clearly a fresh definition or role-up of costs, manpower, contingency, and management procedures will need to be carried out.

As TESLA moves toward becoming an international project, we recommend consideration of:

- contingency in the cost estimate,

- manpower in the cost estimate (although this will be dependent on country),

- choice of project tools and level of implementation consistent with the project size, and

- not completely freezing the project base line until major international players are

on-board. (This was a major mistake made at the SSC.)

\section{TESLA TDR Scope, $500=>800 \mathrm{GeV}$}

The design and cost estimates presented in the TESLA Technical Design Report (TDR) are based on the $500 \mathrm{GeV}$ collider configuration $(250 \mathrm{GeV}$ x $250 \mathrm{GeV}$, TESLA500). This is the "baseline design" for which TESLA believes the technology is in hand. In addition, the TDR "assumes that TESLA will be built from the very beginning with superstructures and cavities reaching gradients of average $35 \mathrm{MV} / \mathrm{m}$, thus allowing a total collision energy of $800 \mathrm{GeV}$." (TDR I-34,35) The plan is to install RF cavities and couplers with capability of operation at $800 \mathrm{GeV}$ within the TESLA-500 cost estimate. Within the "500 GeV" cost envelope higher energy operation at lower luminosity would be possible without upgrading the RF or cryogenics. Operation at $\sim 750 \mathrm{GeV}$ appears possible at $\sim 6 \times 10^{33}$ luminosity. Additional AC power and utilities, RF systems (klystrons, modulators, and cables), and cryogenics plants could be later added to reach $800 \mathrm{GeV}$ capability at high luminosity without extensive civil construction. The ability to explore energy higher than $500 \mathrm{GeV}$, at low luminosity should allow for a sampling of the physics potential without additional investment. Then, if there is an indication of physics, further investment could be made. 
Realizing the $35 \mathrm{MV} / \mathrm{m}$ goal for $800 \mathrm{GeV}$ is the ambitious goal of the ongoing R\&D. Cryomodules and cavities of this gradient do not exist at present and module tests at this gradient may be some years away. We note that recently a 9-cell cavity has achieved $35 \mathrm{MV} / \mathrm{m} \mathrm{CW}$, an encouraging result. Even so, one might expect that were the project to proceed ahead, cavities with the best gradient at the time above the $500 \mathrm{GeV}$ level would be installed with the expectation that gradient improvements would continue throughout the construction phase. We note that the steps to project start will take some years under the most optimistic of schedules. It makes no sense to put the start of these approval procedures in series with achieving solid $35 \mathrm{MV} / \mathrm{m}$ production capability. It would be better if the cavity $\mathrm{R} \& \mathrm{D}$ and project approval process proceed in parallel.

An increase of energy beyond $800 \mathrm{GeV}$ is not contemplated and would require either a different technology (higher accelerating gradient) or a different site layout. For the Hamburg site, an extended layout is not under consideration. Also it has been assumed that substantially higher energy would have to be achieved with a different technology, and most likely at a different site.

The TESLA site design is limited at the southeast end by the city of Hamburg and the deep Elbe River, but is unlimited to the northwest, except for the Baltic Sea Canal (which is a considerable distance away and under which a linac tunnel could be installed). Extending the layout would involve moving the IP point and a number of other tunnel features like the position of the positron damping ring. In order to maintain the possibility of colliding TESLA electrons with $930 \mathrm{GeV}$ protons stored in HERA, and to most fully utilize existing DESY infrastructure, the DESY site was chosen as the southeast terminus for the linac. If another site were chosen (e.g. a U.S. site), issues of site length and layout would doubtless be reopened.

\section{TTF Status and Goals}

\section{a. TTF Cost and Effort Reference}

The total non-manpower materials \& services $(\mathrm{M} \& \mathrm{~S})$ cost for TESLA R\&D (to create TTF1) to date is 45 million Euros from DESY and an additional 13 million Euros from collaborating institutions. Manpower is estimated at 550 man-years from DESY staff (approximately 70 full time equivalents per year over the project time scale) and 400 man-years from collaborating institutions. The ratio of M\&S for TTF (as listed above) vs. FEL elements has been about $75 \% / 25 \%$. This vigorous R\&D program has put the collaboration in the position of having considerable experience with prototype fabrication and systems operation.

\section{b. Priority Technical TTF R\&D Status and Goals}

Gradient Performance and Cryomodule Operations 
The TESLA Test Facility, TTF, has successfully operated for approximately 12,000 hours. Since October 2001, typical up time for 15 weeks of continuous FEL operation has been $>90 \%$. Flat top gradient duration has been between 800 microsec (60\% of the time) and 400 microsec (40\% of the time). Gradients based on FEL requirements were $18.5 \mathrm{MV} / \mathrm{m}$ (module ACC 1 ) and $11 \mathrm{MV} / \mathrm{m}$ (module ACC 2).

The FEL operation at $\sim 250 \mathrm{MeV}$ established laseing in the vacuum ultra violet (VUV) region at wavelength of $80-120 \mathrm{~nm}$. A power gain of $10^{7}$ and a peak brilliance of about 8 orders of magnitude higher than that of $3^{\text {rd }}$ generation light sources were observed. These impressive results help establish the viability of the XFEL proposal.

Experience with operation at higher gradients is now being given high priority. Prior to October 2001, ACC 1 was run above 20MV/m (20-22) for $235 \mathrm{hr}$ with 800 microsec flat top and $1 \mathrm{~Hz}$ rep rate. Since mid-March, 2002, the plan is to run ACC 1 at high gradient. The gradient achieved after a few days is $21 \mathrm{MV} / \mathrm{m}(165 \mathrm{MeV}$ energy gain for the module) with 800 microsec flat top and $5 \mathrm{~Hz}$. The plan is to increase the bunch train from 80 to 800 microsec (with care toward beam transmission) and to run until May 2002. Current R\&D efforts will include extended operational experience in TTF at high gradient. Future plans include installation and test of a new module for high gradient operation. Within TTF, there is continuing priority competition between operation for linear collider validation and for FEL application. The shutdown for TTF II installation will create a major pause in the module testing program. However it is planned to test three new modules for TTF II in spring 2003. Commissioning of TTF II is planned for late summer 2003. It would be more optimal if an alternate test facility were available for further module operating tests. This could be a priority goal for U.S. collaborators.

Continued cavity R\&D toward realization of $35 \mathrm{MV} / \mathrm{m}$ is the major priority. As noted above, one 9 cell electropolished cavity has reached $35 \mathrm{MV} / \mathrm{m}$ in a recent $\mathrm{CW}$ vertical dewar test. The first test of a full cryomodule (\#6) with electropolished cavities is scheduled for early 2004.

The German Science Council recommended that high priority be given to the realization of operation at the gradient goal of $35 \mathrm{MV} / \mathrm{m}$.

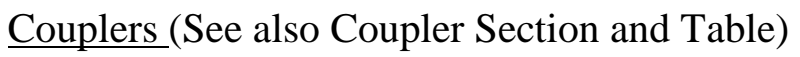

The cavity RF input couplers are as critical a component as the cavity itself. Four different evolving prototype designs have been tested to date. Two for these designs (Fermilab and TTF II) are presently installed in TTF operating modules.

Breakdowns in cryomodules appear to almost always occur at the coupler. After two years of operation the FNAL coupler is limiting the gradient of one of the modules (ACC2). It is believed that this is because of a combination of reasons related to the vacuum on the warm side of the coupler. The module has not been warmed up for considerable time (about 2 years). Whether a warmup and reconditioning of these couplers would restore their power capability has not been tested.

Tesla Engineering Study Review 
High gradient operation of ACC1 (discussed above) with TTF II coupler will be key to testing coupler limitations as well as cavity limitations. The TTF II coupler has been tested on the warm test stand to $1.8 \mathrm{MW}$ of traveling wave power (the test was terminated because of time limitations, not coupler limitations). This is sufficient power for operation of an individual 9 cell cavity at the $800 \mathrm{GeV}$ level. Superstructure coupler design is underway. It is to be noted that superstructures give only marginal beam energy increase. It could however make for considerable savings in coupler and RF distribution costs. For TESLA construction, the coupler costs must be substantially reduced from present.

Tests of the new modified configuration of HOM couplers to damp the $2.585 \mathrm{GHz}$ mode are scheduled for TTF in 2002.

\section{$\underline{\text { Superstructure }}$}

The superstructure concept of connecting two or more cavities with one coupler is in principle a way to improve the active gradient filling factor of the linac. The TDR says this would be a $6 \%$ improvement and the number of couplers would be reduced by a factor of 2 for the $2 \times 9$ cell scheme. It is planned that a superstructure will be tested in TTF during the summer of 2002 and HOMs will be measured. Plans are going ahead to facilitate processing $2 \times 9$ cell, and input coupler prototyping is underway. There is considerable work to be done in order to validate this configuration. Only time will tell if the approach is truly a good idea. It is part of the R\&D program toward $800 \mathrm{GeV}$ capability.

\section{Comments on Technical Accelerator Design}

The basic scope of this study was the five subsystems with largest cost estimates. Purposely, we did not try to study the accelerator physics design (which could be a separate study activity). During this study, some of our group have noted that two of the large subsystems can not really be completely tested or verified before actual construction. The large damping rings and positron source are non-standard and innovative. We did not investigate these sub systems. Alignment throughout the accelerator under the influence of ground motion also came up. We suggest that careful attention be given to the accelerator physics of these systems and that care be given to understanding the operational coupling of the positron source with the rest of the complex. These technical questions are to be addressed by the International Linear Collider Technical Review Committee (ILC-TRC). 


\section{Operability and Reliability}

\section{a. The One Tunnel Concept}

The one tunnel design concept has impact on many of the Main Linac systems and on operability and maintainability. Some of these issues have been discussed in the RF section. Klystrons can only be changed out on maintenance days. A few days per month will be required for tunnel access. Modulators in service buildings are connected to pulse transformer/klystrons by long high voltage pulse cables. Low-level RF, local controls, diagnostics and instrumentation all must be located in the linac tunnel. These systems are inaccessible during RF or beam operation and may need protection from radiation damage. Fire safety is an additional concern that is being addressed in the Planungfeststellungverfahren.

Though a two-tunnel layout would undoubtedly cost more in tunnel and civil engineering cost, it might have major benefits as far as component layout and facility operability/maintainability. Modulators of a more compact design might be located in the tunnel. Not the least of these advantages might occur in the commissioning stage. We believe that a careful study including life cycle considerations of the pros and cons of one- vs. two-tunnel configurations should be carried out to determine the most advantageous choice. Certainly if a TESLA Linear Collider were to be considered for siting in the United States (and possibly in a deep tunnel), a review of this question might assign different relative weighting and optimization to reliability, safety, and cost, leading possibly to a different conclusion.

\section{b. Additional Technical Considerations for Reliability/Operability}

We note here some additional technical areas of consideration, which have come up during this study:

\section{i. Module testing}

During the most active installation period, $75 \%$ of the cryomodules will be installed in the tunnel without test. Possibly there will be cavities with low performance. What is the strategy for dealing with this? What is the expected impact?

\section{ii. RF power budget}

There is a budget for RF losses and regulation (see RF Sections IV.B.1. and IV.B.2.). We did not discuss this budget, operational margins, and their adequacy. However, it is clear that sufficient RF contingency is one key to reliable RF control and operation.

\section{iii. Vacuum failure of the beam tube}


Potential beam tube vacuum failure has always been a significant worry because of the possibility of contamination of large lengths of superconducting cavities. The TDR design has remote-operating vacuum valve isolation every $2.5 \mathrm{~km}$. (In HERA bore tube segmentation is $1.6 \mathrm{~km}$ and has not cause operational problems.) A memo (TESLA Report 2002-06) is beginning to address the vacuum failure issue. Protection from the beam making a hole in a cavity is key and a main function of the Machine Protection System (see below). Other failure modes include coupler ceramic leaks (manageable with careful venting), equipment failure (including common mode failure, e.g. global timing), and human error (from experience this is considered to have the highest potential for a major error). A model for what happens if a hole is produced is being developed. This is a cryogenic question as to how helium will enter the beam tube and become distributed. The driving pressure is only 30 mbar. The model must take into account the expected area of liquid helium coverage before corrective action can be taken. This is an example of on going work to understand the damage extent and will be coupled with review of valve segmentation spacing. It is possible that additional segmentation with remote actuating valves that operate both warm and cold, every $150 \mathrm{~m}$ (cryosector) would be very beneficial. We encourage experiments and actual failure tests as well, to make sure the process is indeed understood.

\section{iv. Insulating vacuum failure or leaks}

The insulating vacuum has vacuum barriers every $500 \mathrm{~m}$, and the modules have manual warm bore tube valves on either end of each module. Thus during installation or repair, module bore tubes can be checked individually but the insulating vacuum must be checked in $1 / 2 \mathrm{~km}$ sections.

Leaks or failures in either the beam tube or insulating vacuum require warming up of $2.5 \mathrm{~km}$ and this is expected to require up to a month for repair of a straight-forward fix. Whether this is the optimal segmentation and its impact will need to be reviewed in the future. (In HERA $1.6 \mathrm{~km}$ sections are warmed up at a time. This is done every year or two for maintenance. Failures requiring special warmup have been few or non-existent.

\section{v. Machine Protection System (Beam Interlock System)}

It is clear that a sophisticated machine protection system will be essential to protect against beam induced bore tube failures. Prototype systems have been or will be implemented in TTF1 and 2. Many of the requirements for such a system have been specified already. These include: monitoring of magnet excitations, valve and vacuum status, loss and BPM monitors, RF, cryo, and water ready, kickers operational, damping ring ready, selection of type of operation, etc. Some of these requirements will be more difficult to determine than others and will require testing at low beam power before full beam operation. The assumption is that all hardware will be checked before beam is injected into the linac. Once in the linac, beam cannot be interrupted (worst case time of flight) for 100 microsec. Fast loss monitors may be of most use to monitor halo, dark current and locate weak cavities. The logic and implementation of this system will doubtless evolve over the duration of the project as potential failures or weaknesses are 
uncovered. For injection into the linac, collimation protection is planned. At the end of the linac, a fast kicker system will be able to deflect beam to an emergency dump. In the linac itself, failures of devices have been considered. The shorting of one pole of a quad will still result in beam staying within the aperture. Steering correctors do not have full aperture strength. Cavities decay slowly if the RF trips, etc. Even so, this will clearly be an area of continued study and failure mode analysis. The present TTF system uses PLC's. For TESLA it would be necessary to set trip window values from the control system when operating conditions change. In conclusion, it is clear that there will be on going studies as to possible failure modes, determining the optimum protection system, and balancing between too much and too little protection. Working on the logic of the machine protection system is an excellent area for collaborator involvement.

\section{vi. Controls System}

While TTF uses an in-house system (Distributed Object Oriented Control System), its applicability for TESLA will need to be reviewed (especially in terms of its extendibility). We note however that there are a number of years between now and operation and that control technology will likely change considerably in the meanwhile. This is an area where the international community could work toward what a future system might be like. It is unlikely that any existing system will be appropriate. Work could begin on specifying desirable features of controls as well as the development of the Global Accelerator Network (GAN) ideas.

\section{vii. Reference RF System}

During our study, questions have been raised about how the reference RF would be distributed, and its stability. First we must note that from beam loading measurements the cavity phase relative to the beam can be determined. This is a very important feature of the superconducting cavities so local corrections can be tracked. But there must be beam. A reference system, which gets the cavity phases close to correct, will be important. An RF reference distribution system probably can be implemented that maintains stability to $+/-20 \mathrm{deg}$. Beam is then used to get 1 degree (at $1.3 \mathrm{GHz}$ ) short and long term stability. The present idea incorporates 3 integrated concepts: $9 \mathrm{MHz}$ will be distributed along the linac to supply local oscillators; calibration will be obtained with the beam; and a fiber optic system will be compared and calibrated against all the cavity measurements. This is a concept where more work certainly will be needed. A first step would be development of a hardware and beam commissioning plan which addresses the needed requirements of the overall low level RF system and how the system gets turned on. Such discussions are only just beginning. This is an excellent area for further collaborator involvement.

\section{viii. Design of installation for modulator power cables}

Modulator cables have been discussed in the RF section. Here, we will only note that they appear to be a considerable logistics problem in the tunnel, both in terms of installation and their failure modes. Cabling systems with access points every $2.5 \mathrm{~km}$ 
must be carefully planned in the tunnel design phase to facilitate necessary repairs or upgrades. A first pass understanding of the cable plant, including spares, cable lengths, installation and cable removal should be done. We note that it is unclear if there really is sufficient space for cables needed for the $800 \mathrm{GeV}$ upgrade. (The initial Fermilab construction installed power cables that were underrated with regards to heat extraction. These cables have caused continued problems over the years. It is often very difficult to rectify deficiencies in the original installation.)

\section{Codes and Standards}

The process for projects done in Germany includes adherence to several codes and standards. The tunneling and below-ground facilities are subject to approval by the Oberbergamt (Bureau of Mines). Above ground construction and infrastructure are subject to various ISO codes. The technical equipment is subject to the ADMerkblaetter/AD2000 (new European Union pressure vessel code) and to the W-10 code, which is similar to ASME, and includes lower temperature properties. The TüV (Society for Supervision of Technical Items) has requirements which apply to various kinds of equipment including pressure vessel, welding additives, and safety valves (including two phase systems).

German codes and safety standards are very equivalent to U.S. codes and standards. However details and the method of meeting these requirements may be different. For example, Fermilab has Oxygen Deficient Hazard (ODH) policies which mitigate the risk of fatalities in the case of release of cryogens (Helium, Nitrogen, etc.) TESLA has minimized these hazards by choosing to not have any liquid Nitrogen below ground. Based on tests performed for HERA and the HERA ODH assessment, DESY does not believe further mitigation is necessary. We recommend that this be further reviewed (see Cryogenics Section).

\section{The Process Used for the TDR Cost Estimates}

The review of the TDR cost estimating in this study report is not intended to be a validation of the costs. Rather, it is intended to examine the process by which the costs were estimated, for example to identify what items are included in the cost estimate in the TDR, and to identify how the estimates were done.

\section{a. Initial Guidance in Preparing the TESLA TDR and Cost Estimates}

In commissioning the preparation of the TDR and its cost estimate, the TESLA management specified that the design of systems and their cost estimate be sufficient for $25 \mathrm{MV} / \mathrm{m}$ operation of all systems, and that the cavities and input couplers (i.e. things inside the modules) be capable of $35 \mathrm{MV} / \mathrm{m}$. The overall design was driven by technology choices to produce reliable operation, and not by minimum cost. The technologies recently developed for TTF would be scaled to the TESLA project. 
We believe there were only minimal trade-offs between initial capital costs and lifetime (operating, maintenance, and repair) costs. Specifically overall electrical power consumption was limited to acceptable level (low compared with other LC's). There were no formal value engineering reviews, but there were internal project reviews and iterative $R \& D$ prototyping.

\section{b. Cost Basis of Major Subsystems, and Series Prototyping}

Costs of major subsystems reported in the TESLA TDR were based on industrial studies conducted over six months. Several institutes were involved in cost evaluations. A planning group supervised layout of production and test facilities for mass production. The experience of many labs has been factored in. In particular the synergy between cryogenics, superconducting cavity and magnet expertise from the HERA project at DESY has been of great benefit to the TESLA project.

Significant cost reductions have been realized through several iterations of prototype TESLA components.

\section{c. Component Cost Estimating}

The TESLA cost estimates are based on TTF R\&D experience and industrial studies. The estimates were based on scaling of costs of few components into the massproduced quantities required. In some cases mass production methods were applied. TESLA management expects the costs to be conservative. They expect a few \%, plus or minus uncertainty on each item, which are expected to balance out, hopefully to a plus $0 \%$, minus a few $\%$, over the entire project. These are bottom-up cost estimates. In particular, no engineering contingencies were allowed to be included. The cost of manpower used by industry in producing their deliverables was included in the cost estimates. However, the 6933 person-years laboratory manpower requirement for DESY and collaborating institutions was included as a separate manpower estimate, and not included as a cost within the 3.1 B Euros project estimate.

TESLA management related only one instance where a top-down cost estimate was invoked for a major component. This was for the new RF tuners and their mechanism. The old design took too much space between cavities. The price for a tuner of a new design was set to the price of the old tuner.

\section{d. Involving Industry}

TESLA has worked extensively with industry in the R\&D phase and in performing mass-production industrialization studies and in producing the cost estimates. In fact, TESLA expects that the cost estimates will be very close to the final contract bids. They have worked hard to pre-qualify multiple vendors on each sub-task. To be eligible for a TESLA contract, it is envisioned that a company would have to be either pre-qualified by participation in the $R \& D$ phase or to have successfully completed a small pre-production run before being awarded the final contract The idea is to develop 
trust in the low-bid company. It is intended that the contracts (if there are only minimal cost disadvantages) will be let to more than one company. We believe this is both good politics and allows a fall-back position. If one company has a major problem, their fraction of the overall task could then be re-assigned to other companies. There will also be the implied threat that, "TESLA will do it themselves."

\section{e. Cost Scaling from a Few to Production Quantities}

Although cost estimates of most items were based on bottoms-up industrial studies, TESLA applied reality checks to these projections by using a quantity scaling algorithm based on industrial experience. The general form of the accumulated average unit cost (what would be the average cost if one had to produce a large number of items) as a function of $\mathrm{N}$, the number of units produced, was assumed to follow the form $\mathrm{C}_{\mathrm{N}}=\mathrm{C}_{1} * \mathrm{f}^{\log \mathrm{N}}$ where $\mathrm{C}_{1}$ is the cost of producing the first items in a small pre-series, and the factor $\mathrm{f}$ varies between 0.75 and 0.85 depending on component.

It is noted that this TESLA formula with $\mathrm{f}=0.8$ (e.g. for 20,592 RF cavities) is similar in behavior to the well-documented "Learning Curve" model with a slope of $93 \%$. A 93\% Learning Curve means that there is a reduction in unit cost of $93 \%$ with each doubling in quantity, for example the $1000^{\text {th }}$ unit costs $93 \%$ of the cost of the $500^{\text {th }}$ unit. (See references at the end of this section.) We note that NLC scales costs according to $85 \%$ - 90\% learning curves (a larger reduction for various mechanical components.

These simple formulations and their characteristic parameters cannot be taken too literally. Their projections depend critically on the particular process and will vary component to component. The TESLA costing was not based on this scaling; it was used only as a check.

It would seem that the uncertainties in the projected cost estimates themselves are larger than the differences in the TESLA and Learning Curve parameterizations.

\section{f. Number of Vendors}

A single vendor model was assumed for the costing exercise. That is, that major contracts like the cavity production, module assembly, or tunnel construction would be let to one company only. These companies would of course have their mixture of suppliers. This assumption simplified the cost studies, that otherwise might have gotten into more complicated scenarios of company size and ability to do part or all of production, procurement strategy, etc.

During our study there has been considerable discussion on this model, if it were to actually be used. Points, issues and strategies that have been discussed include:

- Considerable effort is going into trying to engage and qualify multiple vendors for the large cost high technology TESLA systems. This should make for competitive bidding by vendors TESLA has confidence in and experience with. 
- TESLA states that selection of vendors (one or more) would finally be based on cost and minimization of risk.

- What is the impact of more than one vendor on the cost? Naively from "learning curve" arguments having more vendors might be more costly, if for no other reason than fewer units are produced by each supplier. Start up and infrastructure costs might be higher. But on the positive side for multiple vendors, smaller size contracts may bring in more interested companies that may have the infrastructure on a smaller scale and be more interested in a fraction of the work.

- Risk is a big issue with only one vendor. Here there was considerable controversy. We did not agree with the TESLA position, but as one will see below, this is probably a mute point.

- It is believed that the single vendor model helps assure more competition between vendors and will result in better bids if winner takes all. This feeling is based to a large extent on recent CERN experience. (CERN has very special governance so it is not clear to us just how relevant their experience is.)

- International collaboration is basic to the TESLA project. That collaboration will probably result in multinational sharing of responsibility of the large cost high technology components. Thus the one vendor model is probably mute, and should be treated as nothing more than a model for straightforward cost estimating. Actual procurement strategies will come later.

At this stage, TESLA is developing multiple vendors for many components in order to obtain competitive bids. It is worrisome to TESLA that, for at least some elements, they are working with a sole vendor, with either the technical ability to meet specifications, e.g. RRR 300 Niobium, or experience in the R\&D phase, e.g. $10 \mathrm{MW}$ multi-beam klystron tubes, or the economic interest to participate at this time, e.g. modulators.

TESLA management prefers to concentrate on single companies to provide a particular service or component. This is a means of reducing costs such as non-recurring engineering expenses. The learning curve model mentioned above would imply (for a 93\% model) that the average unit cost produced by a single vendor would be $93 \%$ of the average unit cost for a model where two vendors equally shared the production.

In actual practice, it is not clear if one vendor is most cost efficient. This will depend on production time schedules and available infrastructure at individual vendors. More vendors may be more cost efficient because they may be able to produce at a lower rate with existing or less costly infrastructure. Though TESLA favors a single vendor model, they are willing to consider multiple vendors provided this does not incur substantial cost penalties.

Experience at CERN with the LEP project has shown that having two or three vendors for components, e.g. cryogenics, was a saving grace to getting that project completed on time and on budget. HERA had two dipole suppliers. One of the concerns of the TESLA RF group for example, is the lack of variety of producers of components and systems.

Tesla Engineering Study Review 
We believe that a one-vendor model, although adequate for cost estimating purposes, will not be good practice for the project where there will be too much risk, in terms of both cost exposure and schedule slippage. There will be a need to involve more than one company in specialized areas in order to support the overall industrial base. (See Cryogenics section.)

Most importantly, working out details of international commitments will probably require completely re-evaluating procurement strategies.

\section{g. Financing and Profit Basis}

During the study, there was discussion as to whether profit had been included in the industrial cost studies or not. There were ideas put forward as to possible other ways to provide companies with benefits possibly in lieu of monetary compensation. These ideas were surprising to us. Here we will attempt to clarify our understanding.

Most cost estimates did indeed include profit. The two industrial studies that apparently did not were those carried out by Babcock-Noell for the production of the cavities and modules. (See Section IV.A.1. Cavities). Here, the company chose not to discuss profit to Company A or B and had not included it in their estimate. Profit for subcontractors were included, as these estimates was based primarily on quotations. TESLA believes that of order 5\% profit margin would be more than sufficient. They believe this order of cost could be made up by the project supplying pre-financing for infrastructure and startup, so no correction was made. We are not in a position to assess these complications.

As described above, TESLA discussed infrastructure financing and profit models that are creative and could reduce cost. These ideas may not be relevant in the U.S. Many companies would have to acquire the infrastructure to be able to produce TESLA components. In order to attract companies, TESLA is considering no-interest loans or up-front infrastructure funding. It is also considering transfer of ownership of TESLA financed infrastructure at the end of construction, allowing a company to use its new expertise and infrastructure for its own endeavors. Just what cost advantages these concepts would have is unclear to us.

\section{h. Personnel Cost Estimating}

The personnel cost estimate in the TDR is 6933 person-years which includes the following activities at the TESLA site: design, procurement, follow up of fabrication, QC, system assembly, testing, installation and hardware checkout and (pre-beam) commissioning. This total does not include TTF R\&D or other pre-project costs. It does not include beam commissioning, pre-operating efforts, or manpower overheads for administration. The manpower estimate was based on HERA and TTF experience at DESY. We note for comparison that LEP and LHC each estimated less than 5000 manyears laboratory staff effort.

Tesla Engineering Study Review 
At DESY, the conversion number for the cost of manpower is $\sim 50 \mathrm{~K}$ Euros per person-year on the average. For German industry, this manpower cost is $\sim 60 \mathrm{~K}$ Euros per year. This may be compared to the $\$ 83 \mathrm{~K}$ per person-year, on the average, used at Fermilab. We note the favorable manpower rates in Germany relative to the U.S. or Japan. This can have a significant impact when considering the relative costs for projects around the world.

It is very hard to know if 6933 man-years is a reasonable estimate without a detailed study. It was stated by TESLA leadership that approximately $50 \%$ of the estimated laboratory manpower effort would be for tunnel installation and hardware commissioning. It is likely that some of this labor force would include contract labor.

\section{i. Installation Costs}

For projects installed in the U.S., a fraction of the installation costs would be covered under the Davis-Bacon law. There would need to be a high-tech/low-tech determination whether specific tasks could be accomplished by the industrial/union workforce, or would required skilled laboratory employees. In any case, the average cost (salary plus fringes) per union worker in the Chicago area is not very much different than the average cost per Fermilab employee.

As examples of some of the labor categories, we list the following: Technical coordination and administration (EDMS, coordination, civil engineering, and safety) is estimated at $\sim 540$ man-years. Physics support is $~ 500$ man-years. By far the largest effort demands are in the RF and vacuum systems at approximately 1300 and 1000 manyears, respectively, including component testing, installation, and hardware commissioning.

\section{j. Component Testing}

The largest component testing cost element will be discussed as an example of how testing would be done. This element includes the superconducting RF cavities and the cryomodules (cryostats) for the Main Linacs.

TESLA is planning to test $100 \%$ of the RF cavities in a vertical dewar before assembly into the cryomodules. This qualifies the cavities at the earliest time in production, and could allow for the option of sorting and grouping of cavities of similar performance, which will improve the overall performance of the linac. Initially, every one of the assembled cryomodules will be tested at DESY before installation. This will be during the production ramp-up phase where testing can keep pace with production. Once confidence is reached that operational quality cryomodules are being consistently produced, the testing rate is anticipated to drop to approximately $25 \%$. This will occur when the production rate exceeds the rate capacity of the envisioned test facility. It is estimated that $\sim 300$ man-years will be devoted to this effort (71 people for 4 years plus startup). It is under consideration to commission an industrial study to determine, given 
the expected failure rates and consequences, whether this $25 \%$ testing level is the appropriate goal.

\section{Differences in Cost Estimating Between the TDR and U.S. Practice}

Here we identify several items which are handled differently in U.S. DOE-style and German-style cost estimates for a project such as a Linear Collider. We note that the so-called "European cost estimate" is not a uniform standard and varies from project to project. We close this section with a rough comparison of ongoing TESLA activities relative to DOE critical decision points, and an attempt to map the TDR costs into a U.S. type framework.

\section{a. Contingency Accounting}

In Germany, projects are expected to be completed on or below budget and on or ahead of schedule. The proponents are expected to have done all of their homework, including working with industry on realistic cost and schedule estimates, before submitting the proposal. The successes of PETRA and HERA give the TESLA group confidence in the estimates for the TESLA project. Nevertheless, recent experience with larger projects, such as CERN's LHC, raises concern as to the prudence of proposing an international project of the scale of TESLA without explicit inclusion of contingency.

In addressing the issue of the required magnitude of contingency, the DESY Director pointed out that PETRA at DESY was built at a cost of 98.5 MDM and within budget. And HERA was built at a cost of 1.372 B DM, with a $2.8 \%$ overrun. HERA's overrun was absorbed within the normal DESY operating budget. It is to be noted that the current understanding of the design and hardware of TESLA is ahead of that of HERA at a comparable stage. For that matter, the TESLA modules are probably further advanced than LHC magnets at its project start.

We learned that on the German project forms, there is no element for including, or requesting contingency funding. The lack of added contingency in the TESLA cost estimate is based on the assumption that the R\&D and industrialization studies have been mostly completed on the large cost items, and that further R\&D and competitive bidding will result in decreased production costs, thereby generating some contingency "margin". The costs for the tunnel, buildings, and utilities/infrastructure are also considered to be well understood for construction in the Hamburg area. There are no surprises anticipated for these elements. However, details of the complete design have not been worked out at this stage, and contracts have not been let. There undoubtedly remain some technical components which still have technical uncertainly. Without a contingency reserve, any overrun would have to be absorbed in the future operating budget, schedule slippage, reduction in scope or capability, or in a request for supplemental funding. 
A contingency reserve and contingency management throughout the project would appear to be sound and conservative project procedure. Flexibility in managing changes is essential for efficient adjustment for on-going developments. If the project does not need to use its contingency, or uses excess contingency to provide additional scope, it is all the better. The percentage allotted should depend on perceived risk. Certainly any U.S. commitment would contain contingency.

We list below examples of accelerator projects both in the U.S. and in Europe and their contingency history (to the best of our knowledge). Initial contingencies as a percentage of cost to complete are listed.

\begin{tabular}{|c|c|c|}
\hline Project & Initial Contingency & $\underline{\text { Total Cost (incl. Contingency) }}$ \\
\hline FNAL (200 BeV) & $32 \%$ & $\$ 243 \mathrm{M}$ final cost. \\
\hline SSC Lab (TEC, SCDR 7/90) & $18.4 \%$ & $\$ 5,913 \mathrm{M}$ initial baseline \\
\hline Advanced Photon Source & $22 \%$ & \$ 459 M initial cost est. \\
\hline RHIC & $14 \%$ & $487 \mathrm{M}$ final cost \\
\hline PEP-II & $23 \%$ & $177 \mathrm{M}$ final cost \\
\hline Fermilab Main Injector & $21 \%$ & $230 \mathrm{M}$ final cost \\
\hline CEBAF & $20 \%$ & $\$ 313 \mathrm{M}$ final cost \\
\hline Spallation Neutron Source & $17.6 \%$ & $\$ 1,293 \mathrm{M}$ initial cost est. \\
\hline ESRF & $5 \%$ & $880 \mathrm{M} \mathrm{CHF}$ \\
\hline SPS* & $12 \%$ & $1,780 \mathrm{M} \mathrm{CHF}$ \\
\hline LEP* & $-0-$ & 1,294 M CHF (18\% overrun) \\
\hline & \multicolumn{2}{|c|}{ ( $12.5 \%$ contingency added at 1986 mid-project re-baseline) } \\
\hline LHC* & \multicolumn{2}{|c|}{ (18.3\% increased baseline, under discussion, Nov. 2001) } \\
\hline HERA* & $-0-$ & 1,372 M DM (2.8 \% overrun) \\
\hline
\end{tabular}

The LEP construction experienced an overall $18 \%$ overrun relative to the initial cost estimate. This overrun was distributed as $-2 \%$ machine components, $59 \%$ machine infrastructure, $17 \%$ injector systems, $18 \%$ underground civil construction, and $67 \%$ surface civil construction. (CERN/FC/3313, 1990)

* We note that the projects denoted with an asterisk * did not include laboratory personnel in the project cost. Some overrun in both LEP and LHC is attributable to lack of laboratory personnel to fill the project demand. Also, we note that if internal laboratory budgets do not include personnel costs, it is inherently more difficult to move people to areas of higher priority and resource demand.

ESRF is interesting because it was green field construction. The total cost included manpower. There was contingency. The civil construction was $\sim 25 \%$ of the total and $8 \%$ of this was for an AECM contract. 
Thus we can see that although there is a perception of a "European Cost Model", actual projects differ one from another.

\section{b. Schedule, Scope and Technical Contingency}

\section{Schedule}

As noted earlier, concepts of installation, commissioning and associated schedules and required manpower are at a rudimentary planning stage. The level of development is probably appropriate for this stage in the project. Detailed plans often are not worked out until well into the project and production schedules are set. Inevitably, industrial problems will translate into TESLA cost and schedule problems. We note that there appears to be little explicit schedule contingency built in and schedule risks have not yet been explicitly analyzed.

A slight cost over-run could likely be absorbed within the operating budgets of DESY or the institute of that project task. Larger over-runs would likely require additional project funding.

\section{Scope contingency}

The possibility of scope contingency was briefly discussed. We note that, given budget problems, for a linac, there is always the possibility of reducing the top energy by building fewer cavities and RF systems, whereas this is not an alternative for a circular accelerator. In the U.S., the top-level project scope specification is clearly defined at the time of base lining and scope reduction would require difficult negotiating, unless it could be shown to have little physics impact.

Technical Contingency (operability, maintainability, failure modes)

Technical contingency has been included in some areas (e.g. additional linac as a buffer for the anticipated klystron failure rate during operations). This margin can as well be used to cover high power and low-level RF system failures. Extra RF stations are planned for the injector linacs. Tunnel power supplies have spares. Also $50 \%$ extra capacity for the cryogenics plants has been included. But a full investigation of areas where technical contingency is desirable and possible has yet to be carried out. Technical contingency is strongly related to operability, maintainability and failure modes as well. At this stage in the project, it is probably reasonable that thorough studies have not been carried out. However, we recommend that such investigations proceed in the near future. This is an area for collaborative effort. 


\section{Project Implementation, Management and Cost}

\section{a. Current TESLA Proposal for Project Management}

The TESLA project organization is envisioned to consist of countries or institutions operating as a partnership of limited-liability shareholders. The partners would elect a supervisory council that directs and manages the project. The partners would contribute components or subsystems (as part of their own national programs). The facility would be the common property of the participating countries or laboratories, which would also share in the responsibility and cost for operation. Each collaborator would be funded, reviewed and managed by its own country and responsible for its own overruns.

DESY's confidence in being able to manage its part of the project is in part based on the successes of the PETRA and HERA projects. These were financed primarily by the German Federal Government and the State of Hamburg (78\% in the case of HERA), and received additional in-kind contributions of hardware from collaborating countries. It is intended that TESLA scientists and engineers would manage this project.

\section{b. Civil construction}

There would be no overall AECM (Architectural Engineering and Construction Management) firm, in the U.S. sense. The plan is to put great reliance on "design and build" contracts, which transfer much of the AECM effort to the contractor. A small engineering management group, working with TESLA, would then oversee the work. Presently the firms of Windels, Timm and Morgen, and Amberg Ingenieurbüro have been working under contract with DESY on the TESLA conventional construction conceptual designs, specifications, and Planungfeststellungverfahren. Three other firms are involved as well. The cost is $\sim 1 \mathrm{M}$ Euros/yr for about 10 FTE's of effort. Such firms would likely provide construction management as well. About 3\% of the civil construction budget is devoted for these services. For comparison, about $7 \%$ of the HERA civil construction costs were for AECM, CERN had approximately $3 \%$ (CERN staff costs) for managing the design and build tunnel contract for LEP, but Fermilab had $\sim 4 \%$ internal staff and $\sim 14 \%$ external contract costs for AECM activities on the conventional construction for the Main Injector. This \% difference possibly indicates the advantages of "design and build" over conventional U.S. procedure where a separate firm prepares the design review and construction packages, and supervises the construction contractor.

The tunneling, anticipated to be the largest single contract, would be handled as a Design and Build contract. This approach would also apply to the conventional buildings and utilities (power and water), as well as possibly even to the cryogenics plants. This approach minimizes the amount of construction engineering needed on the project by TESLA staff, while reducing costs by integrated optimization of the design relative to the construction capabilities of the contractor. It is noted that for the HERA tunnel, the winning contractor, a joint venture headed by Wayss \& Freytag, submitted a superior 
proposal based on different means and methods than originally planned by Windels and Timm (Tunnels and Tunneling, January, 1986, pages 26-29).

\section{c. Project Management Tools \& Systems Engineering - Management Information Systems}

TESLA is now testing commercial production scheduling software EDMS (Engineering Data Management Systems) which has typically been developed for, and used by, auto manufacturers. This system has strong potential for use in cavity production, for instance. It is recognized that an additional system will be needed to bridge between this and the financial SAP package in order to support scheduling, procurement, cost, and earned-value management.

During the few years of most intense activity, the TESLA manpower time profile shows approximately 67 full-time equivalents per year (FTEs) for technical coordination administration (civil engineering, safety, coordination, and EDMS). This amounts to about 500 man-years, about half of which is devoted to EDMS tracking.

We believe that it is very important to develop to the appropriate level project management and systems engineering tools and procedures as much as possible before construction starts. Getting the optimum level of implementation to make these tools most useful without undue overhead is the issue. Use of these tools at the appropriate level will surely benefit such a large project as TESLA.

\section{d. Administrative Overhead}

Administrative overhead is not included in the project cost estimate. It is estimated that there would be another average of $\sim 87$ FTEs, summed over DESY and collaborating institutions, added for business, administration, and bureaucratic overheads. This administrative overhead represents $10 \%$ of the technical manpower invested in the project $(10 \% * 6933 \mathrm{M}-\mathrm{yr} / 8 \mathrm{yr})$ and is comparable to the administrative to technical ratio at DESY today. TESLA's share of the total overhead expenses or the accounting for the infrastructure costs, including manpower for administration, services, computing, library, security, site maintenance, etc., is estimated to be $\sim 25 \%$ (35 M Euros per year) of the continuing DESY operating budget.

\section{e. Environment, Safety and Health (ES\&H)}

As mentioned above TESLA is presently preparing a Planungfeststellungverfahren. This documentation and process are similar to that required for an environmental assessment in the U.S. It is the complete legal basis for construction permission. It includes exact definition of the site layout, and addresses environmental concerns (geology, water, spoils, smog, etc.), public concerns (traffic, line sag on the electric power grid, etc.), public and worker radiation and safety, tunnel safety (fire), safety during both construction and operation, etc. 
DESY intends to submit this plan for review by the authorities in summer to fall of this year (2002). It is hoping for authorization to proceed with the public hearings phase of the environmental process by the end of the year.

Clearly TESLA already is proceeding with various ES\&H activities that normally would come later in the DOE project approval process after site selection. (A more complete comparison of the maturity of the TESLA project compared to DOE Critical Decisions is given later in this section.) TESLA costs do include ES\&H provisions required by German law. U.S.-specific ES\&H specific requirements, of course, are not considered in the TESLA TDR.

\section{f. Pre-Operations Costs}

Pre-operations costs are not included in the TDR. In DOE cost accounting, these costs would be included in the Total Project Cost (TPC) but not in the Total Estimated Cost (TEC). Thus, the TESLA cost estimate is best compared with a TEC estimate (see Below).

An example of pre-operations costs would be that for the cryogenic systems operation during the commissioning phase for the entire machine. We note that personnel for commissioning is included in the estimate as well as helium inventory but operating costs are not. It is expected that pre-operating costs will be supported by the on-going DESY budget. The TESLA TDR estimates that operating expenses will be $120 \mathrm{M}$ Euros/yr when the machine is operating for experimental physics. Pre-operating testing and commissioning costs are not anticipated to be larger than this operating budget.

\section{Comparison of TESLA Project Activities to DOE Project Management Timelines}

It should come as no surprise that the past and ongoing activities of the TESLA collaboration do not map neatly onto DOE project management guidelines, simply because Germany is not bound by those requirements for managing a project. In this section we will attempt to compare TESLA project activities with the DOE project time line. This comparison is given in a Table at the end of this section. The DOE project management timelines can be summarized around five critical decisions that allow the project to proceed and that trigger the accounting of costs for the project.

The critical DOE decision steps CD-0 through CD-4, between phases (and subphases) of a large construction project, such as a Linear Collider project, are outlined below. Note that the Acquisition Performance Baseline is a key element. It contains the performance (scope, key parameters), schedule, and cost, as a contract between the project and the DOE. 
Initiation Phase (Pre-Acquisition and Pre-Conceptual)

CD-0 => Approve Mission Need (by Deputy Secretary of Energy)

Definition Phase (Conceptual Design Phase)

Permits work to begin on pre-project planning activities that will identify products to be provided and requirements to be met.

CD-1 => Approve System Requirements and Alternatives

Execution Phase (Preliminary Design Phase 20-35\%)

Permits commencement of final design activities, studies, specifications, drawings, regulatory permits, etc., initiate long-lead procurement actions. Can request special Project Engineering and Design funds (part of TEC) for preliminary and final "design only".

CD-2 => Approve Acquisition Performance Baseline (request \$ from Congress) (Final Design Phase)

Authorizes final design to proceed, provided funds are available.

CD-3 => Authorization to Complete Implementation

Authorizes Procurement and Construction

Transition/Closeout Phase (Acceptance/Completion)

CD-4 => Approve Project Transition Complete

(Completion of Project $=>$ Begin Operations $)$

The total project cost (TPC) includes the total estimated cost (TEC - specifically funded through the U.S. Congress) plus other project costs (laboratory operating costs for activities such as conceptual design, $\mathrm{R} \& \mathrm{D}$ during project, pre-operations, etc.). The accounting of the TPC begins with CD-0 and ends with CD-4. The accounting of the TEC begins with CD-1 and continues until well after CD-3 when other project costs are used for the transition to operations. For a Linear Collider project, the TEC, which includes the construction and equipment costs, would dominate the TPC. Although the TDR cost estimate does contain the bulk of the TEC, it does not contain as explicit costs all the costs that DOE would account in the TEC, like manpower, overheads, contingency, etc. (See next section)

A brief comparison of TESLA activities with respect to some of these DOE project management timelines is given below. This is not intended to be a comprehensive or exact checklist as to what TESLA has completed relative to U.S. requirements, but rather to indicate the level of progress and formality TESLA has achieved.

The DOE timeline and key requirements are outlined from Section 2.3.1 and Figures 2-2, 3-11, and 3-15 from the exposure draft for the Implementation Manual for DOE Order 413.3. See reference at end of this section.

A few remarks about the TESLA project status in comparison to DOE decision steps are in order. 
The German government has asked the German Science Council to evaluate the TESLA proposal. It is expected that the recommendation from this body will be available by the end of 2002. The government is expected to react to that recommendation in mid2003.

The TESLA TDR and associated cost estimate seems to satisfy many of the requirements for CD-0, namely the performance goals, technical descriptions, and preliminary order of range estimates for the technical scope, schedules, and costs, and a few for CD-1. Notable exceptions are formal analysis of risk and related contingency in the systems engineering sense, and a preliminary acquisition execution plan. CD-2 approval for requesting project funding would require a specific Acquisition Performance Baseline and formal management planning requirements.

It is to be noted that the TESLA Collaboration is already working on elements that would most likely require prior CD-0, or higher, approval for a DOE project. Site selection, environmental assessment, and project base line development activities are all well underway.

We see that TESLA is well into the DOE project Definition or Conceptual Design Phase. It has prepared both conceptual and technical design reports and cost estimate.

TESLA appears to be very close to having determined some elements of the project baseline including site-specific design and environmental assessment. The next hurdle will be encouragement to proceed from the German Government.

TESLA is already preparing advanced designs on some topics such as civil construction and infrastructure. TESLA management intends to pre-qualify companies for the bids on major contracts. These activities will allow them to efficiently award the bids shortly after "project approval" or availability of funding. Even so, it is expected to take about a year after this approval to place the contracts. This "project approval" might be compared to CD-3 in the DOE system.

Exactly how one might map TESLA's status into the DOE requirements, doubtless, is open for discussion. Our attempt is given in the following table. What is clear is that the German system has provided a fast and efficient mechanism for getting to the project decision point, and for preparing to actually move ahead on the project once the go ahead is given.

The German system does require internal laboratory review (Scientific Council), national scientific review (German Science Council), scientific community support (ECFA), and will require international support. However, it appears that many of the checks and requirements of the U.S. system do not apply in Germany and that TESLA is undoubtedly years (or well) ahead in its German approval process in contrast to the situation if it were a proposal for a project in the U.S. 
As TESLA moves toward becoming an international project, a whole new set of negotiations will need to get underway. A question that will come up is the interaction of the international participants in the development of the final project baseline and management structure. We note from SSC experience, that full involvement of international collaborators is necessary at an early stage, and the lead country must be careful to engage partners as soon as possible in both the project planning and governance determination and in the technical decision process.

TESLA is at a stage where it is important to begin to build the governance of an international laboratory. This is well understood by the TESLA collaboration and will be necessary for whatever LC is built. Cooperation from all parties in beginning to discuss these issues will be necessary if a project start of any LC will be realized perhaps as early as 2005 . 


\section{U.S. DOE 413.3 Acquisition Management System Summary Level Processes \& Key Primary Requirements}

\author{
Initiation Phase \\ / Pre-Acquisition and Pre-Conceptual \\ Laboratory Operating Funds (non-TPC) \\ | Req.: Mission Need Statement (goals, objectives, capabilities) \\ Small -Scale Testing \\ Preliminary Environmental Strategy \\ Preliminary Acquisition Plan \\ Integrated Project Team \\ V/ Risk Comparison and Assessment \\ CD-0: Approve Mission Need (Deputy Secretary of Energy) $====>$ \\ / Definition Phase \\ Other Project Costs (part of TPC - Laboratory Operating Funds and Project Engineering \& Design Funds) \\ Conceptual Design \\ Req.: Conceptual Design Report \\ Prelim. baseline ranges for technical scope, schedules, \& costs \\ Identify engineering development vs. proven process \\ Preliminary safety $\&$ hazard studies, analyses, \& documents \\ Complete Proof of Concept Testing \\ Work Breakdown Structure \\ Complete Environmental Requirements \\ External Reviews \\ Assign Project Manager \\ Selection of Alternatives \\ Risk Management \\ Project Assessment and Reporting System (PARS) \\ Project interfaces defined and responsibilities assigned \\ Project Execution Plan \\ CD-1: Approve System Requirements and Alternatives \\ / Execution Phase \\ Total Estimated Costs (TEC Project Funding) \\ Preliminary (20-35\%) Design \\ Req.: Project Execution Plan \\ Project Management System \\ Earned Value Management System > \$ 20 M \\ External Independent Review \\ Acquisition Performance Baseline <= key item \\ Reporting and PARS \\ Performance Verification: \\ a. Full-Scale Tests \\ b. Refinement/Optimization - Engineering Scale Tests \\ * TESLA TDR \\ * TESLA TDR \\ * TDR and TTF \\ * Planungfestellungverfarhen \\ * demonstrated $500 \mathrm{GeV}$ at TTF \\ $\sim$ demonstration of $800 \mathrm{GeV}$ \\ although TESLA would deny it \\ $\sim$ Planungfestellungverfarhen \\ $\sim$ German Science Council \\ $\sim$ \\ $\mathbf{x}$ \\ $\mathbf{x}$ \\ $\mathbf{x}$ \\ $\mathbf{x}$ \\ ? \\ $\sim$ TTF components and \\ conventional construction design \\ CD-2: Approve Acquisition Performance Baseline \\ TEC Project Funding <==> Congressional Authorization \\ Final Design \\ CD-3: Authorization to Complete Implementation \\ V/ Procure/Construct \\ / Transition/Closeout Phase \\ Other Project Costs (other TPC - Laboratory Operating Funding with minor TEC Project Funding) \\ Acceptance/Completion \\ V/ Req.: Project Closeout, Contract(s) Closeout, Lessons Learned Report \\ CD-4: Approve Project Transition Complete
}




\section{Cost Estimate Mapped to U.S. Format}

A major question at the start of this study was how the TESLA cost estimate would compare to an equivalent cost estimate produced in the United States. There are real differences in what is included and what is not included in these two methods of cost estimating. For the sake of a concrete example, we will consider only the estimated capital construction cost of the Linear Collider part of the TESLA project, (without including detector(s) or FEL) which amounts to 3.136 Billion Euros in year 2000 funding. Converting at $\$ 0.90$ per Euro, this estimate becomes $\$ 2.822 \mathrm{~B}$. This is the cost to be paid out by the TESLA collaborating institutions, not including institution manpower, contingency, general and administrative overheads (G\&A), and escalation (inflation) factors which are included in similar U.S. calculations of the total estimated cost (TEC). Note that costs for acquisition of surface land parcels $\left(860,000 \mathrm{~m}^{2}\right.$ or 213 acres), but not underground easements, are included in the TESLA estimate. Neither surface land nor underground easements would be included in a U.S. estimate. Below, we will try to see what the cost might look like with these U.S. inclusions and with U.S. labor rates.

It should be noted that neither the TESLA estimate following the German methodology and labor accounting, nor a U.S. style format with U.S. labor accounting and contingency, attempt to address what might be an international format where many of the differences will need to be worked out in the overall collaboration governance, and partner by partner, nation by nation.

What we attempt here is a comparison of the TESLA estimate as modeled as being built as a German project, with a U.S. style estimate as modeled if TESLA were built as a U.S. project.

\section{a. Manpower}

The manpower from the TESLA institutions for technical coordination, inspection, and administration (EDIA) are included in the project manpower estimate, but not explicitly included in the Euro cost estimate. As TESLA is planned to be an international project with international laboratory personnel involvement, it is more transparent to give the manpower as FTE's (full-time equivalents), not as Euros.

As all of the skilled personnel may not be available from DESY or the collaborating institutions and to map cost to the U.S. method, we translate the 6933 manyears to Euros and \$. Note that there is a higher average cost for labor in the U.S. $(\$ 83 \mathrm{~K}$ per man-year at Fermilab) than in Germany (51 K Euro at DESY and $60 \mathrm{~K}$ Euro for industrial manufacturing rates (technician, craft, laborer)). This difference affects not only laboratory personnel costs, but also is hidden in the cost of purchased materials and services, taken to be about 50\% material and 50\% manpower, on the average. If TESLA, or various components were to be built in the U.S., those purchased items would have another multiplicative factor of $(0.5+0.5 * 83 / 60 * 0.9)=1.27$ to account for the difference

Tesla Engineering Study Review $114 \quad$ July 8, 2002 (final) version 
in the cost of manpower used to produce those items. There would also be a question of how much of the installation work would be covered under the Davis-Bacon act. However, the Davis-Bacon average wages are comparable to the average Fermilab wages.

The industrial manpower rate difference between Germany and the U.S. make for a substantial increase in the project cost. It would appear that value in Germany is considerably better. This is probably an idiosyncrasy of the international monetary exchange rate and not a statement of standard of living at either location.

\section{b. General and Administration}

On a project of the scale of TESLA, the General and Administrative (G\&A) overhead costs would likely be negotiable between each collaborating institution and its funding agency. Using the current Fermilab model, the G\&A costs would be a strong function of the number of large $(>\$ 500 \mathrm{~K})$ contracts and their average cost. A realistic estimate of the G\&A cost depends on the contract procurement strategy and general overhead support function. In the U.S., the G\&A rate is a matter of negotiation, project by project. In this example, we use $30 \%$ overhead on personnel costs and $3 \%$ on materials, services, and contracts (M\&S). This results in $\sim \$ 280 \mathrm{M}$ addition to the cost. As noted above, TESLA expects administrative support for the project to be $10 \%$ of the laboratory effort. Adding in the other overhead functions also described in section F.10.b, TESLA anticipates that the total overhead/infrastructure support rate will require approximately 25\% (35 M Euros per year) of the continuing DESY operating budget, or $280 \mathrm{M}$ Euros over the 8 years of construction.

\section{c. Escalation}

Escalation will be included in the German project at the time of approval. The present estimate is in 2000 year Euros. For the U.S., a model might be $2 \%$ per year with a mid-construction average of year 2007. This gives $15 \%$ escalation or $\sim \$ 670 \mathrm{M}$. This escalation factor is also applied to the TESLA estimate for this example.

\section{d. Contingency}

In the U.S., contingency would be applied based on perceived technical and cost risk. Without that detailed analysis, for this example, we choose to apply a somewhat arbitrary $20 \%$ based on historical experience for other projects (see table in section IV.F.9.a) at the time of their initial project data sheet or base lining. It is also recognized that at this level of understanding of the TELSA cost estimate (before base lining), some might have chosen to apply a larger (30-40\%) contingency for this illustrative example. For TESLA based in Germany, the recommended amount of contingency might be

considerably lower, were contingency to be accepted as a concept. We note the success of ESRF project with a modest contingency. For an international project, contingency will be a matter for overall project governance and also for the individual collaborators to address in their specific commitments. 
The following is a very informal model showing representative elements of a U.S. project cost estimate. This is specifically not an estimate of what TESLA would cost to be built in the U.S. Such an estimate would have to be very site-specific including geology, land/easement acquisition, and existing infrastructure issues. U.S. industrial cost estimates would need to be obtained. It more closely represents what it would cost, in our traditional accounting terms, for U.S. institutions to build TESLA-500 at the Hamburg site, not including the extra travel, housing, and administration costs of working so remotely from the U.S.

We see that in U.S. terms, the quoted cost estimate of TESLA might be about a factor of 2 greater than the TDR cost estimate. This, in itself, is not surprising. What is interesting is the major impact of the difference in labor rates and the inclusion of the cost of laboratory labor. These labor cost differences, when G\&A, escalation, and contingency are applied result in $\sim \$ 1.5 \mathrm{~B}$ of this $\$ 3 \mathrm{~B}$ difference! 


\section{A Re-Maping of the TESLA Estimate to a U.S. Style Format and Labor Rates}

This is not a validation of the TESLA estimate nor an estimate of what TESLA would cost if built in the U.S.

\section{TESLA Project Cost Estimate Comparison}

Base cost estimate - from TESLA TDR

(to be paid out to industries and companies)

U.S. industrial manpower cost factor of $27 \%$ of $\$ 2.822 \mathrm{~B}$

Personnel Costs from TESLA Institutions - 6933 man-years

European (51 K Euros/yr)

U.S. (\$ $83 \mathrm{~K} / \mathrm{yr})$

EDIA: Included in Institutional manpower

General and Administrative Overheads

TESLA: adds $25 \%$ of DESY operating budget for business, administration and other overhead functions $35 \mathrm{M}$ Euros per year for 8 years

\section{$\underline{\text { European Union U.S. ( TEC) }}$}

3.136 B Eur \$2.822 B

0.354 B Eur

\$ $0.575 \mathrm{~B}$

$-0-\quad-0-$

U.S.: assume model $30 \%$ of laboratory manpower plus $3 \%$ of material and contracts (negotiable)

Escalation (Inflation) - TDR quoted as year 2000 prices

TESLA: to be added at time of approval (assume 15\%) 0.566 Eur

U.S.: Assume 2\%/yr \& 2007 average costing date $\rightarrow$ 15\%

\$0.666 B

Contingency added to Cost Estimate

TESLA: none

-0 -

U.S.: assume $20 \%$, of above sum

$\$ 1.021 \mathrm{~B}$

total estimates

ニニニニニ=ニ=
4.336 B Eur $\quad \$ 6.126 \mathrm{~B}$

European Union $\quad \underline{\text { U.S. }}$ 


\section{References for Learning Curves:}

R.D. Stewart, R.M. Wyskida, J.D. Johannes, Cost Estimator's Reference Manual, Second Edition, John Wiley \& Sons, Inc., New York.

H. Kerzner, Project Management - A Systems Approach to Planning, Scheduling, and Controlling, Sixth Edition, John Wiley \& Sons, Inc., New York.

Cost Estimating Guide, DOE G 430.1-1, 03-28-97, Chapter 21, U.S. Department of Energy, 1997. http://www.directives.doe.gov/pdfs/doe/doetext/neword/430/g4301-1chp21.pdf

C.J. Waterworth, Relearning the Learning Curve: A Review of the Derivation and Applications of Learning-Curve Theory, Project Management Journal, March 2000, page 24-31.

\section{References for DOE 413 Series: Project Management:}

\section{http://www.directives.doe.gov/pdfs/doe/doetext/neword/413/XXX.pdf}

where XXX.pdf refers to:

p4131.pdf Program and Project Management Policy for Planning, Programming, Budgeting, and Acquisition of Capital Assets (Policy)

04131.pdf Management Control Program (DOE Order 413.1)

n4131.pdf Extension of DOE Order 413.1 (Notice)

o4132a.pdf Laboratory Directed Research and Development (Order)

04133.pdf Program \& Project Management for Acquisition of Capital Assets (Order) An exposure draft for Implementation Manual for DOE Order 413.3 is also available at http://www.cfo.doe.gov/oecm/

see also the Glossary of U.S. and DOE Terms and Acronyms in the Appendices 


\section{Summary, Conclusions, and Recommendations}

\section{A. Summary}

\section{This Study}

This study was undertaken to understand the engineering design, status, and costing methodology of the major TESLA systems (Main Linac modules, Main Linac $\mathrm{RF}$, civil construction, conventional and cryogenic machine infrastructure, XFEL). It is not meant to be either a cost validation or a physics study of the technical accelerator design.

\section{History}

TESLA from its inception has been proposed as an international project.

A decade ago when TESLA R\&D began, superconducting RF cavity technology was considered by most people to be too costly, too unreliable, and too low a gradient to be a serious contender for linear colliders. At the same time it was perceived that the low frequency cavities with their large iris diameter would make much lower wake fields resulting in easier tolerance and control of emittance growth. The superconducting cavities in addition have efficient conversion of electric energy to beam energy.

\section{TTF R\&D}

Cavity R\&D was initiated with the goal of reaching $15 \mathrm{MV} / \mathrm{m}$ in TTF and proving feasibility of $25 \mathrm{MV} / \mathrm{m}$ for a TESLA-500 LC. Gradient operation in TTF is in excess of $20 \mathrm{MV} / \mathrm{m}$ and cavities in modules yet to be installed, on the average, exceed gradients of $27 \mathrm{MV} / \mathrm{m}$.

\section{Status for $500 \mathrm{GeV}$}

The baseline design of the TDR is $500 \mathrm{GeV}$. It appears that the basic technology is well in hand for TESLA-500. This study concludes that the cost of the superconducting technology no longer is a major issue in determining the technology for a Linear Collider. The substantial reduction in superconducting RF costs per MV over that in existing projects reflects: the cost effective design of long cold module strings, the dramatic increase in operating gradient, and economy of mass production.

\section{TESLA-800}

Major cavity R\&D activity is directed toward the goal of obtaining $35 \mathrm{MV} / \mathrm{m}$ production cavities over the next few years so that were the project approved, high gradient cavities and power couplers could be installed from the beginning. The goal is to try to install $800 \mathrm{GeV}$ cavity capability at the outset. RF power and cryogenic upgrades 
necessary for high luminosity $800 \mathrm{GeV}$ would come later, though initial operation up to $700 \mathrm{GeV}$ would be possible at lower beam current and luminosity.

We note the recent successful test of an electropolished 9-cell cavity to $35 \mathrm{MV} / \mathrm{m}$ $\mathrm{CW}$, the first 9-cell TESLA cavity to reach this gradient. (Other cavities have operated above $34 \mathrm{MV}$ in pulsed mode.)

We note that the superstructure is also part of the $800 \mathrm{GeV}$ plan. We do not consider this as fundamental or important a development as achieving the high gradient.

\section{Progress to Date and Future}

The TESLA Collaboration is to be congratulated on the dramatic progress that has been made to date. The progress on cavities and operation of TTF has been outstanding. The technical design of the Linear Collider with cost estimate and site consideration has been carried out. The project has been proposed to the German government. There still is much to do in cavity $\mathrm{R} \& \mathrm{D}$, module life testing, refinements of the accelerator design, engineering systems, project logistics, etc but the basic proposal and proof of principle has been carried out very effectively.

We note as indication of systems status that TTF has operated with two cryomodules for about 12,000 hours. A beam of $\sim 250 \mathrm{MeV}$ has been supplied for FEL development and the FEL has demonstrated saturation in the $100 \mathrm{~nm}$ wavelength region.

We believe that the TESLA technical status is at a stage consistent with submission of the project proposal to the German government for response in 2003.

\section{Priority}

The German Science Council in its review of the project recommended that high priority be given to the realization of operation at the gradient goal of $35 \mathrm{MV} / \mathrm{m}$. The TESLA Collaboration has always realized the importance of this priority and is proceeding. We certainly second this goal. It will undoubtedly be a key discussion point when international project participation is initiated.

Though recent emphasis has been directed toward steps required to obtain German government approval, future emphasis will be directed toward further development of the international laboratory project structure. 


\section{B. Findings and Recommendations}

\section{Project Overview}

\section{a. R\&D Iterative design}

Many systems are evolving through a series of prototype designs or improved procedures. These iterations are directed toward simplified design, improved technical performance, and reduced cost. This is in a sense a direct method of "value engineering" that we believe will clearly benefit the project. The more R\&D that can be carried out the better the final engineered system.

\section{b. Industrial Studies of the Manufacturing Process and Cost Estimate}

Industrial studies were contracted to analyze the manufacturing process, determine a possible production procedure with necessary infrastructure and resources and estimate the costs. In particular, detailed studies were done by at least two separate firms on the cavity manufacturing and on the cavity processing/module assembly. We believe this provides a level of confidence in the estimates beyond what would be achieved by laboratory personnel.

\section{c. One Vendor (Contractor) Model}

Cost estimates were based on a one-vendor model for each large procurement. This was a simple approach to the costing exercise. The strategy that is underway calls for developing and qualifying as many vendors as possible for the high technology critical systems. (We note that there are three qualified cavity manufacturers.). Qualified vendor competition would then be assured for the production and the one vendor selection would probably result in obtaining best offers. Besides assuring competition, the single contractor model could well be less costly if based on volume cost reduction and single infrastructure investment.

We believe that the one vendor approach would not be realistic during the project. It entails both too much risk and does not give economic incentive over a broad enough industrial base. Consequently, the estimates of cost and risk may be optimistic. We realize that strategies for obtaining best proposals are complicated and can only be seriously addressed in the project.

However most importantly, we note that for an international project, multiple vendors may happen naturally.

TESLA is trying to broaden the number of vendors qualified on major components during the R\&D phase. This is one important area of possible collaboration now. 


\section{d. Industrial Profit}

There was considerable discussion over profit and if it had been properly included in the estimate. Confusion arose because the industrial studies for cavity fabrication and module assembly did not include profit and the company was unwilling to supply that number. We believe that other studies and estimates all have included profit. In dealing with the profit issue for the cavities and modules, TESLA management realized that up front pre-financing by the TESLA project to provide for infrastructure and pre-operation costs could well save sufficient cost to cover the profit expense. In conclusion, no additional cost (expected to be at most 5\%) was added to these two elements.

\section{e. Manpower}

Laboratory (and collaborator) manpower is estimated at 6933 man-years. About half of this is for installation and hardware commissioning. (Beam commissioning is not included in the project cost or effort.) This manpower could be contract people as well as laboratory employees. It is impossible to know if this is reasonable without further study.

The cost of manpower was not included in the TESLA estimate as an explicit number in Euros, but rather it was expressed as needed FTEs (full-time-equivalents). This was because of two reasons: first, the German project system does not usually include the cost of laboratory personnel in the estimate; second, for an international project where laboratory resources will come from many international partners, a cost expressed at the German labor rate has less meaning than the needed head count.

Even so, as TESLA looks more into the international aspects of the project, we recommend that the cost of this effort be included in the project cost. This gives a better perspective of the overall project magnitude and overhead requirements. The information of required manpower resources need not be lost. Also experience shows us that Laboratory personnel are not always available in which case the effort becomes a cost expenditure to the project.

\section{f. Manpower rates}

Based on present exchange rates, German manpower rates are low compared to comparable U.S. rates. This has a significant impact on overall project cost if one tries to estimate what the project would cost in a U.S. framework as compared with a German framework. A very rough model gives a $\$ 1.5 \mathrm{~B}$ estimated increment just for adjustment of labor rates with associated overheads (G\&A, escalation, contingency) on the labor differential. This one factor goes a long way to explain the differences in the estimated costs that we arrive at when comparing the overall cost including manpower of the TESLA project in Euro (4.05 B Euro) with the estimate using U.S. practices (\$ 6.1 B). (See section $\mathrm{m}$ below) 


\section{g. Schedule, Installation and Hardware Commissioning}

Development of resource loaded installation and commissioning plans are only beginning. This is to be expected at this point of project planning. We recommend that the installation/commissioning plan including beam commissioning be further developed in order to identify needed procedures and to see just how reasonable the schedule and manpower estimates actually are. There is no explicit overall schedule contingency identified. (This is an area for potential future collaborative effort.)

\section{h. Technical Contingency}

As the goal of TESLA is to install cavities with gradient capability above that needed for the $500 \mathrm{GeV}$, there is in that sense built in contingency for the baseline. There is a small amount of excess LINAC to allow for klystron and RF system failure during operation. However the potential for technical contingency in other areas still needs further study, as well as investigation of operability/maintainability, risk and failure modes. Examples noted are: strategy for dealing with weak cavities once they are installed, does the RF power budget have enough reserve? Will the low level RF reference system be stable against long term drifts? What will be the effect of vacuum failure of the cavity beam pipe? What is the effect of halo and dark current? Further specification of the machine protection system is needed, and what beam failure modes will or will not be protected against? What failure modes may result in cavity damage? etc. We realize that work is ongoing in many of these areas, and encourage further development of analysis and test to assure sufficient technical contingency, operability, and risk abatement. (This is an area for potential future collaborative effort.)

\section{i. One Tunnel}

The TESLA Main Linac (including other beam transport systems) has a onetunnel layout with all necessary distributed systems located in this one tunnel. This layout has significant impact on the engineering component configuration (especially the RF system) and on the availability/maintainability of systems. The installation and maintainability of the large number of modulator cables required needs further study, especially for the $800 \mathrm{GeV}$ upgrade where we suspect there may not be sufficient tunnel space. The arrangement of tunnel components has recently been optimized allowing for the installation of all $800 \mathrm{GeV}$ cables. The safety and radiation damage aspects of one tunnel as well as the cost increment of two tunnels are, of course, significant considerations as well. We believe a study of the pros and cons of one vs. two would be very helpful in clarifying the tradeoffs. If TESLA becomes an approved international project with an international scientific program, the impact of this choice will need to be analyzed. Were TESLA to become sited in a different location (country) the tradeoffs between cost, safety requirements and maintainability might well be different. 


\section{j. Administrative Support and Laboratory Overhead Functions}

Administrative support and Laboratory overhead functions are not included in the project cost estimate. TESLA estimates that these functions will require $\sim 25 \%$ of DESY's operating budget.

\section{k. Project Management Tools and Systems Engineering Methodology}

Tools such as EDMS are presently under evaluation. It is recognized that considerably more work must go into the area of management tools. We strongly encourage effort in this direction. Tools that are understood and can be put in place before project start are of the greatest value. It is extremely important to search for the optimum level of managing, reviewing, resource scheduling, and forecasting. These are tools developed to be useful but the project must choose a course that applies to it neither too little nor too much - in a way to be most effective.

\section{Contingency}

The TESLA cost estimate does not include contingency as a separate line. The German project system does not accommodate it. However there is considerable evidence that it is prudent to have available contingency funds for unexpected and unforeseen developments. For TESLA as an international project, we recommend the inclusion of contingency. The amount chosen will be that which seems most appropriate when reviewed case by case for risk.

\section{m. Project cost - German / U.S. comparison}

We have tried to make a simple model of cost comparison. This model is not to be taken too seriously. It certainly does not represent a cost review or validation but rather an approximate remapping of the TESLA costs into U.S. terms (specifically TEC). We find that the 3.1 B Euro of the TESLA basic cost becomes 4.3 B Euro when laboratory manpower, administrative manpower, and escalation are added. Not added are other overhead functions and any contingency. For the U.S. mapping, we have used a simple approximation for the difference in labor rates in the two countries, and applied this approximation to both industrial and laboratory manpower. We included a model for General and Administrative Overhead (G\&A), then added escalation and contingency. The cost estimate in U.S. terms would become $\$ 6.1 \mathrm{~B}$. Of this estimate $\sim \$ 1.5 \mathrm{~B}$ is related to the labor rate difference assumed and associated G\&A, escalation and contingency.

Needless to say, neither model is really all that relevant, they just set a comparative scale. A Linear Collider will not be built as a national project, either in Germany or the U.S. It will be international and with different countries responsible for different systems. Costs to the various countries may be either more or less expensive than assumed here for either mapping. Exchange rates will have little impact on in kind contributions. 
We do believe and recommend that in the international context, contingency reserve be included and managed by the international project management group, as well as by individual partners.

\section{n. Process to Project Approval}

We have tried to compare the TESLA activities to date that have been needed to get a proposal before the German government and to position TESLA to move ahead should approval be granted, with U.S. guidelines. We find that the German procedures to project start appear to be more time efficient and more pragmatic and lead more quickly to a project decision. We believe that a TESLA Project in Germany is considerably ahead of a comparable project in the U.S., even if the same technology is used.

\section{o. Project Status - Baseline}

It is clear that the status of TESLA as a German project is approaching the point of baseline definition. The preparation of the Planungfeststellungverfahren (a project description for environmental and safety assessment) is underway. This document is the complete legal basis for construction permission. There is the hope that the plan can be put before the public in 2003 .

\section{p. Toward an International TESLA Project}

The TESLA collaboration for R\&D has been international from its inception, and there are many areas where further assistance from collaborators now would allow for greater progress and engagement in the technical decision process.

Steps toward seeking approval for TESLA in the German system are well along. The German government is expected to react to the recommendation of the science council by 2003. What is less clear is how to proceed (and on what time scale) to turn TESLA into an International Project with international commitment. Once the German government response is known, if it is favorable, then the building of the international community will become critical.

Here, there is a conundrum. We worry that if the project scope and baseline are too frozen, there will not be opportunity for international groups to interact in major technical choices. On the other hand, TESLA planning and technical choices must proceed ahead if progress is to be made. 


\section{Cavities and Modules}

\section{a. Niobium}

There is one major supplier of high RRR material and it is in the U.S. Cost of the material is a significant part of the cavity cost. It could be beneficial if U.S. collaborators could work with this U.S. company to reduce expected cost.

\section{b. Cavity R\&D}

Cavity R\&D has been and continues to be the critical area of TESLA. The present goal of $35 \mathrm{MV} / \mathrm{m}$ is of first priority.

\section{c. Superstructure}

The superstructure with one coupler per two cavities is a goal for TESLA-800. Though this does give some energy benefit and may reduce cost because of the reduction by a factor of two of the number of couplers, flanges, and number of units to be processed, we do not find this direction as compelling and necessary as the cavity gradient R\&D.

\section{d. Cryomodules and $12 \mathrm{~m}$ vs. $17 \mathrm{~m}$ modules}

We note the potential for further mechanical design advances in the areas of flange connection, interconnect regions, coupler bake out, and module transportability.

We do not find the differences between the $12 \mathrm{~m}$ and $17 \mathrm{~m}$ lengths of great significance. We note that the longer length may require larger shafts. We did not review the cost impacts.

\section{e. Couplers}

Input couplers continue to remain a key risk component. A number of prototypes have been developed and tested with good success. More and longer testing is needed.

\section{f. Module Life Tests}

High priority should and is being given to module tests at their maximum operating gradient. This tests both cavities and couplers. There is the worry that the planned shutdown for TTF II installation will limit module tests at a critical stage. Every effort should be made to see that module testing can proceed as much as possible. This is an area where system testing by collaborating institutions could be of considerable benefit. 


\section{g. Vacuum Failure}

Accidental rupture of the cavity bore tube vacuum has always been a considerable worry. A study is ongoing to understand just exactly what would happen if a hole were made in a cavity. We encourage this study and believe that a test experiment should be developed to check out predictions and resulting deterioration of gradient performance.

\section{h. Alignment and Tunnel Motion}

During our discussions on module assembly, it was not completely clear if all parties understood all the necessary alignment specifications. A review of this subject and/or clear specifications might be valuable. Of particular note are systematic errors of one or more modules and the relative role of quad and cavity errors.

We believe a review of potential long-term tunnel motion and how it would be handled could also be of value.

\section{RF Systems}

\section{a. Klystron tube lifetime}

The assumed average lifetime (40,000 hours) of the new klystron tube has not yet been independently analyzed, and meaningful life testing takes years. If the lifetime would be lower, then there would be a commensurate penalty in commissioning time, operating costs, and integrated luminosity (uptime). We recommend further independent analysis of the expected lifetime and failure modes, and a study of possible design changes to improve the expected lifetime as suggested by Thales.

\section{b. R\&D on modulators}

Both TESLA and our study group believe there is potential for further R\&D on the modulators and their controls and interlocks. This could save on size, cost and complexity. We recommend review of the need for a modulator backup switch.

\section{c. Modulator Cables}

As noted above the one tunnel arrangement has significant impact on the RF power system layout. The modulator cables are not an attractive feature.

\section{d. Low Level RF System}

The low-level RF system is by necessity complicated. We believe there is significant R\&D engineering work still to be done in this area and that sufficient manpower should be assigned. We agree with the TESLA LLRF designer that expert engineering on specification development and design for production is critical at this 
stage in order to have a solid production system. This is an area where collaborator assistance would be very welcome.

The stability of the reference RF along the Linac to temperature variations was questioned. We note, however, that because of the superconducting cavities, beam loading will give a clear calibration signal at individual cavities. We suggest that this question of RF phasing and reference phase be reviewed. We note that a commissioning plan for both hardware and beam would help clarify what will actually be needed and that further collaboration here would be helpful and educational.

\section{Civil Engineering}

\section{a. Design \& Build Contracts and AECM}

We note that TESLA does not account for an external Architectural Engineering Construction Management group responsible for construction design and review and contractor management. Instead they rely on Design Build Contracts where the builder is also responsible for carrying out detailed design. The result is that the engineering construction group is much smaller and responsible for limited conceptual design and construction oversight. In the TESLA estimate $\sim 3 \%$ of construction cost is budgeted for this activity, whereas a typical AECM percentage would be $20 \%$. This difference also may be due to the additional formal review processes required in U.S. government construction. We recommend that serious consideration be given to the Design-Build concept in the U.S. for construction of this type.

\section{b. Necessary construction}

The TESLA conventional construction includes only items that are absolutely necessary. It is assumed that office and support space (except for the Module Test Facility) requirements exist at DESY and are not part of the project scope.

\section{c. Construction cost comparison}

We find that for conventional construction, there is considerable difference between the TESLA estimate and an estimate done for an NLC sited at Fermilab (this factor of $\sim 1: 2.5$, respectively). Most of this difference is not in the base numbers of tunnels and technical buildings, but in add-ons, such as campus buildings, AECM, contingency, etc. We direct the reader to Section IV.C. for further details of a comparison between a comparison of construction costs between TESLA and a U.S. type estimate. 


\section{Cryogenic Plants and Distribution}

\section{a. Cryo Plant and Distribution System Cost Basis}

The cryo plant costs are based on recent DESY and LHC studies and experience, academic studies, industrial design and cost studies, and budget quotations. We noted that interconnect of refrigerator components might not have been included in the estimate, but were assured that it had been covered. Compared to the LHC layout, the TESLA refrigerator components are situated very close together, so the cost of interconnections between the various components are relatively smaller than for LHC.

\section{b. Cryo Plant Technical Contingency}

The cryo plants have 50\% technical contingency since the plant capacity is designed to be $50 \%$ larger than the anticipated load for TESLA-500.

\section{c. Process Cycle Program}

We believe that a process cycle simulation program would be of significant benefit for simulation of the integrated plant and module system. Such a program should be flexible enough for application in other similar refrigeration systems.

\section{XFEL}

\section{a. XFEL Sharing Part of the Electron Linac with the Collider}

The TESLA TDR proposal includes an XFEL concept that shares part of the electron linac up to $50 \mathrm{GeV}$ energy. Beam would be extracted at $\sim 20 \mathrm{GeV}$ and at $\sim 50$ $\mathrm{GeV}$ points and would then be transported in a separate line in the linac tunnel to the FEL beam lines and experimental areas. This arrangement strongly couples the operation of the LC and the FEL. Beam pulses would be interwoven and there is a long transport to the beamlines. The estimated incremental cost to the accelerating systems is $242 \mathrm{M}$ Euro in addition to the LC estimate. This assumes construction at the same time as TESLA LC so as to benefit from the large procurements.

\section{b. XFEL Using an Independent LINAC}

Since the publication of the TDR, an independent linac for the FEL has been studied. This would be built along with the TESLA linac at its high energy end and would share utilities. Additional costs would be associated with $10 \%$ extra linac modules, RF systems, and infrastructure. The resulting XFEL cost is estimated at $464 \mathrm{M}$ Euro (the above $242 \mathrm{M}$ for integrated increments plus $222 \mathrm{M}$ for an independent linac) incremental on the LC estimate, again, if built contiguous with the collider. We believe there may be the possibility of further energy optimization, and suggest consideration of energy lower than $50 \mathrm{GeV}$. In addition it may be useful to consider what is the optimum depth of the 
stand alone XFEL. Since the TDR this XFEL layout has been accepted as the preferred plan and review of design features as proposed above is on going. 


\section{R\&D Opportunities}

TTF R\&D has made tremendous strides over the past decade. We believe TESLA has established the superconducting RF approach as a serious linear collider technology.

The U.S. TESLA Collaboration (Argonne, Cornell, Fermilab, JLab, and UCLA) has been involved in TTF R\&D providing both expertise and hardware for superconducting RF cavities, photo-injector components, modulators, RF couplers, cryogenic components, and undulator vacuum chambers. These U.S. TESLA Collaborating Institutions and individuals are listed as authors on the TESLA TDR. Other U.S. Institutions not currently part of the U.S. TESLA Collaboration (such as SLAC) also may have significant expertise to offer. In recent years, because of external constraints, the U.S. collaboration with TESLA has continued at a much reduced level. TESLA welcomes increased international collaboration and, in fact, needs it in order to carry out the multitude of tasks necessary in preparing for such a big international project. Such international participation can be in proof-of-principle R\&D, component testing, qualifying local national industries and vendors, and reduction of project risks and costs. Some examples of such areas are given below. Throughout the text other examples have been noted.

modulators - optimization and investigation of alternative technologies, and design of cost effective control and interlock, review of need of backup switch;

vendor sources - developing national vendor source(s) - for klystrons, bare RF cavities, RF couplers, cryomodules, modulators, etc.;

Niobium - working with suppliers of high purity Niobium, in the U.S. and elsewhere, to understand and try to reduce cost;

cavity R\&D - for very high gradient, high Q proof of principle;

module engineering - improved flange and interconnect designs, transportability;

low level RF systems - work on the design and prototyping of LLRF systems and frequency reference;

FEL - providing components, such as vacuum chambers, and undulators, which also would have direct application to national FEL programs;

positron production - testing of concept of positron production (both non-polarized and polarized), and helical undulator development;

fast kickers - prototype development of damping ring injection and extraction kickers; this is an area of R\&D that would benefit all LC options; 
accelerator controls - work on software and the design of the Global Accelerator Network;

injector systems - development of electron guns, both polarized and non-polarized, 3.9 GHz cavities for long low space charge bunches, flat beam development (high emittance aspect ratio);

beam dynamics studies - both theoretical and experimental, such as coupling, space charge, and instabilities in damping rings and component alignment, stability, ground motion compensation, etc.;

operational studies - such as commissioning scenarios, failure analyses, lifetime analyses;

life testing - module and RF systems testing and operation.

In addition, TESLA has proposed to provide a $1.3 \mathrm{GHz}$ cryomodule to the Fermilab-Northern Illinois Photoinjector Lab (FNPL) to upgrade its energy capability and to acquire experience in installation and operation of these systems.

As members of the international TESLA collaboration, several U.S. groups, both from universities and national laboratories, have been playing an active role in the development of the project. As outlined at several places in the report, there exist many opportunities for additional R\&D, which would be helpful and needed to further optimize various aspects of the design. This work should continue to be done within the framework of international collaboration.

The U.S. HEPAP Subpanel has recommended that the highest priority of the U.S. Program be a linear collider, wherever it is built in the world. They emphasize the importance of making an early technology choice, and recognize that this will require a focused and intensified R\&D program, which must be given very high priority.

TESLA is clearly at a stage where the concepts of what makes an international laboratory, and what are its governance and political outreach structures need to be further developed. These are difficult problems that any LC will need to address along with the need for wide international participation. Developing these ideas needs to proceed in parallel with technical development. 


\section{Appendices}

\section{Charge for this study:}

\section{TESLA Engineering Study/Review (Draft - HTE 9-20-01)}

\section{Goals}

1) Understand the TESLA Cost Estimate, what was done, its structure, basis, scope, and methodology.

What is the overall scope of work for the main individual estimate elements?

What is included (not included) in the estimate?

How was the estimate obtained?

Understand industrial studies, schedules, and supporting documents compiled by TESLA.

What were the technology choices and optimizations?

Are there maintainability issues?

2) Understand the overall project scope, integration, schedule, logistics, etc. (TESLA Project Overview Group)

Both Overview and Individual Element Groups should:

Review manpower estimates, schedules, production, test, and installation plans, and their phasing in time. What type of manpower is assumed for what effort?

Understand areas where estimates may contain contingency (safety factors) or where different approaches or R\&D are expected to lead to cost reduction.

Review manpower assigned for project management, engineering design, inspection, administration, accelerator physics support, construction management, etc.

Overview Topics:

TTF costs vs. TESLA costs - scaling factors used

Company profit? Financing? Investments and factories constructed?

Use of Project Management Firm?

3) Undertake a re-mapping of the TESLA information into US context to reflect differences in approach between standard US costing procedure and the procedures followed by the TESLA collaboration for the specific Hamburg site.

Compare TESLA construction estimates with Fermilab construction estimates performed for near surface and deep sites for the NLC.

This study, or subsequent studies, could analyze the impact of surface or deep site on the cryogenic design and cost.

The FEL layout may be re-evaluated with reference to a FNAL site.

Subsequent studies could analyze the impact on conventional construction for

Tesla Engineering Study Review 
different layouts (surface and deep) of the TESLA Collider, the XFEL, and the Experimental Halls and Facilities.

EDIA, AECM, Project Management, G\&A, and Contingency costs and effort should be explicitly addressed and mapped from the TESLA estimate.

SWF costs per man-year should be estimated.

$\mathrm{R} \& \mathrm{D}$, pre-operating costs, and detector costs could be addressed.

4) Identify (but do not investigate) possible alternative options and R\&D which might be interesting to develop because of their merit or because of US specific and industry context.

\section{Procedure}

Working groups and group coordinators will be appointed in the following areas:

Construction (tunnel, shafts, buildings, site, infrastructure, power, water)

FEL

Cryogenics Plant and Distribution

RF Cavities

Cryostats

RF Power (modulator, RF distribution, Low Level RF)

TESLA Project Overview (see item I.2 above)

Working group leaders will contact counterparts in TESLA and arrange for transfer of information either by e-mail, trips, video, or other exchange. It is recommended that as many as possible of those unfamiliar with DESY plan to visit.

\section{Deliverables}

Presentation after initial introduction

Presentations at intermediate point in the study

Presentations at end of study

Write-up at end of study

\section{Schedule}

August-September - Groups get organized, meet, and absorb published info

September $\quad-$ Setup initial contacts, initial scope presentations

October-November - Visit DESY where necessary

November-December - Mid-term presentations and draft reports

January, 2002 - Finalize Report, final presentations (to TESLA representatives also)

V. The Final Report will be reviewed by the TESLA Collaboration Management and U.S. laboratory management prior to final release.

To whom the report will be addressed is currently under discussion.

The goal is to familiarize and acquaint ourselves with the design and cost process.

The activity should be carried out in a collegial atmosphere. 


\title{
TESLA Engineering Study Participants, Working Groups, and TESLA Contacts
}

\author{
Study Leaders: $\quad$ H. Edwards ${ }^{1}$ and P. Garbincius ${ }^{1}$
}

\section{A. Main Linac Components}

1. RF Cavities - H. Padamsee ${ }^{2}$ and J. Preble ${ }^{3}$, leaders; M. Foley ${ }^{1}$, A. Rowe ${ }^{1}$, and J. Noonan ${ }^{4}$; and D. Proch ${ }^{5}$, TESLA contact.

2. Cryomodules - T. Nicol ${ }^{1}$ and J.G. Weisend $\mathrm{II}^{6}$, leaders;

B. Main Linac RF

T. Page ${ }^{1}$, and T. Peterson ${ }^{1}$; and C. Pagani ${ }^{7}$, TESLA Contact

\section{High Power RF - Modulators and Klystrons}

2. RF Distribution and Low Level RF R. Pasquinelli ${ }^{1}$, H. Pfeffer ${ }^{1}$ and D. Wolff ${ }^{1}$, leaders; John Cornuelle $^{6}$, C. Jensen ${ }^{1}$, and G. Pile ${ }^{4}$; and S. Choroba ${ }^{5}$ and S. Simrock ${ }^{5}$, TESLA Contacts

C. Tunnels and Buildings $-\mathrm{V}$. Kuchler ${ }^{1}$, leader; M. Ross ${ }^{6}$; and W. Bialowons ${ }^{5}$, TESLA Contact

D. Machine Infrastructure

1. Conventional Utilities and Systems - V. Kuchler ${ }^{1}$, leader; M. Ross ${ }^{6}$; and W. Bialowons ${ }^{5}$, TESLA Contact

2. Cryogenics - A. Klebaner ${ }^{1}$, leader; J. Theilacker ${ }^{1}$, R. Rabehl ${ }^{1}$, C. Boffo ${ }^{1}$, T. Peterson ${ }^{1}$, H. Carter ${ }^{1}$, and W. Chronis ${ }^{3}$; and S. Wolff ${ }^{5}$, H. Quack ${ }^{8}$, and P. LeBrun ${ }^{9}$, TESLA Contacts

E. XFEL Increments - Y. Cho ${ }^{4}$, leader; and J. Rossbach ${ }^{5}$, TESLA Contact

F. TESLA Project Coordination, Overview, Integration, Logistics, Management - D. Finley ${ }^{1}$, leader; T. Dombeck ${ }^{1}$, P. Garbincius ${ }^{1}$, R. Stefanski ${ }^{1}$, T. Lavine ${ }^{6}$, and J. Delayen ${ }^{3}$; and D. Trines ${ }^{5}$, TESLA Contact

\section{Affiliations:}

${ }^{1}$ Fermi National Accelerator Laboratory, Batavia, IL, USA ;

2 Cornell University, Ithaca, NY, USA;

3 Thomas Jefferson National Accelerator Facility, Newport News, VA, USA;

4 Argonne National Laboratory, Argonne, IL, USA;

5 DESY, Deutsches Elektronen-Synchotron, Hamburg, Germany;

6 SLAC, Stanford Linear Accelerator Center, Stanford, CA, USA;

7 INFN and University of Milano, Milan, Italy;

8 Technical University of Dresden, Dresden, Germany; and

9 CERN, European Organization for Nuclear Research, Geneva, Switzerland. 


\section{References:}

1. The primary reference is the TESLA web page: tesla.desy.de

This contains links to many descriptions, reports, documents, figures, etc.

In particular, the following documents can be linked through this TESLA web page or directly accessed:

2. TESLA Technical Design Report, March, 2001

DESY 2001-011, ECFA 2001-209, TESLA Report 2001-23, TESLA-FEL 2001-05

This report, with links to many contained references, is available on-line at:

tesla.desy.de/new_pages/TDR_CD/start.html

3. A more complete and updated collection of links to all TESLA reports is available at: tesla.desy.de/new_pages/3330_TESLA_reports_pages.html

4. The public information website for TESLA is:

www.desy.de/pr-info/desyhome/html/presse/hginfos.en.html\#tesla 


\title{
Glossary of U.S. and DOE Terms and Acronyms
}

(in common words, rather than DOE-speak)

Acquisition Performance Baseline - a key element in the project approval and authorization process. This defines the performance (scope, key parameters, etc.), schedule, and cost, as in a contract between the project and DOE (funding agency). This forms the basis of the request for funding from the U.S. Congress.

\begin{abstract}
AECM - Architectural, Engineering, and Construction Management. The process of managing a conventional construction process, often contracted to an external AECM firm.
\end{abstract}

CD-0 thru CD-1 - Critical Decisions in the steps of the review and approval process for DOE projects.

Davis-Bacon Act - a U.S. law specifying which construction related work can be done by laboratory (or U.S. government) employees, and which work must be contracted out to the private sector and the minimum salaries to be paid.

EDIA - Engineering, Design, Inspection, and Administration, aspects of a laboratory's staff's efforts in managing a project (which is often implemented by an external contractor).

ES\&H - Environment, Safety, and Health concerns, regulations, etc.

FTE - Full-time-equivalent(s), the number of full-time persons working on a project at a given time.

G\&A - General and Administrative Overheads, the cost of running a laboratory, which is typically added to a project cost. The G\&A rates vary between U.S. laboratories, and are typically different for M\&S and SWF categories. The G\&A rates for major projects are typically evaluated and negotiated at the beginning of each project.

M\&S - costs of Materials and Services generally paid-out by the laboratory to external vendors and contractors.

Mission Need Statement - a short, introductory statement introducing and describing the goals, objectives, requirements, and general capabilities of a proposed project. Approval of the MNS for a project of the scope of a Linear Collider would reside with the Deputy Secretary of Energy.

SWF - Salaries, Wages, and Fringe benefits paid to the laboratory's staff.

TEC - Total Estimated Cost of a project, specifically funded through the U.S. Congress.

Tesla Engineering Study Review 
TPC - Total Project Cost includes the TEC, plus other related costs, typically funded through the laboratory and DOE budgets, which may include conceptual design, initial $\mathrm{R} \& \mathrm{D}$, pre-operations costs, etc.

Value Engineering - an organized effort directed at analyzing the functions of systems, equipment, facilities, services and supplies for the purpose of achieving the essential functions at the lowest life-cycle cost consistent with the required performance, quality, reliability, and safety. 


\section{Element Descriptions for TESLA Conventional Construction and Infrastructure}

\section{Introduction:}

The following descriptions were developed based on general discussions during my visit to the DESY Laboratory. The intent is to create an understanding of the content of the TESLA Budget Book, specifically for the Conventional Facilities that will be provided as part of the TESLA project. A brief discussion of various aspects of the project scope and comments on the cost estimating process are included in the main body of this report.

Work Breakdown Structure (WBS) category numbers, corresponding to elements or entries in the TESLA Budget Book, were attached by Fermilab. This was both to more nearly match customary U.S. formats and to facilitate cross-referencing.

The buildings and tunnel enclosures are identified in TESLA WBS 1.1.3, "Tunnel and Buildings". However, additional items that are considered, from the Fermilab perspective, to be part of "Conventional Facilities" are also contained in TESLA WBS 1.1.4, "Machine Infrastructure" and are identified by element number below.

Note that there are some inconsistencies in the sizes of the surface buildings between the TDR, the TESLA Budget Book, and the schematic table presented at Snowmass in July, 2001.

\subsubsection{Tunnel \& Buildings}

\subsubsection{Tunnels and Access Shafts}

1.1.3.1.1 Main Linac- This is the Main Linac enclosure. It consists of a bored tunnel, fully lined with precast concrete "tubbings". Due to construction below sea level, the tunnel will constructed using a shielded tunnel boring machine incorporating a pressurized cutting head to eliminate water infiltration and a recycled bentonite slurry for the removal of excavated material. This enclosure is $33 \mathrm{~km}$ in length and has an inside finished diameter of $5.2 \mathrm{~m}$. Grouting behind the precast concrete is included and will be accomplished as part of the precast liner installation. This tunnel is intended to be constructed as part of a design/build project with the design costs included in the per lineal meter unit cost. This unit cost was developed based on the actual tunnel costs incurred during the construction of the HERA project. The finished tunnel will consist of the interior surface of the precast lining with no provision for lighting, 
HVAC, electrical distribution, monorail or other interior finishes. (These are included in the 1.1.4 Machine Infrastructure section.)

1.1.3.1.2 Damping Ring - These are four "loops" connected to the Main Linac enclosure. Like the Main Linac, these "loops" consist of a bored tunnel, fully lined with precast concrete "tubbings". Due to construction below sea level, the tunnel will constructed using a shielded tunnel boring machine incorporating a pressurized cutting head to eliminate water infiltration and a recycled bentonite slurry for the removal of excavated material. These enclosures total $4 \mathrm{~km}$ in length and have an inside finished diameter of $3 \mathrm{~m}$. Grouting behind the precast concrete is included and will be accomplished as part of the precast liner installation. This tunnel is intended to be constructed as part of a design/build project with the design costs included in the per meter unit cost. This unit cost was developed based on the actual tunnel costs incurred during the construction of the HERA project. The finished tunnel will consist of the interior surface of the precast lining with no provision for lighting, HVAC, electrical distribution, monorail or other interior finishes. (These are included in the 1.1.4 Machine Infrastructure section.)

1.1.3.1.3 Access Shafts - There are nineteen access shafts included in the TESLA conventional construction of which eleven are permanent shafts and eight are considered temporary for the facilitation of construction activities and will be filled in with no surface access at the completion of construction. These shafts are nominally $15 \mathrm{~m}$ in finished diameter, and range from 20 $\mathrm{m}$ to $30 \mathrm{~m}$ in depth. They are to be concrete structures, either cast-inplace or precast lined depending on the final contracted design. In the estimate, a single average unit cost per shaft has been assigned. These shafts are intended to be constructed as part of a design/build project with the design costs included in the unit cost per shaft. The shafts were sized at 15 meters, but new cryomodules are planned to be 17 meters long, requiring larger access shafts to prevent having to tip the cryomodules during installation.

1.1.3.1.4 Positron By-pass Tunnel - This is the enclosure that allows the positron beam to by-pass the interaction region and be transported into the positron Main Linac tunnel. It consists of a bored tunnel, fully lined with precast concrete "tubbings". Due to construction below sea level, the tunnel will constructed using a shielded tunnel boring machine incorporating a pressurized cutting head to eliminate water infiltration and a recycled bentonite slurry for the removal of excavated material. This enclosure is $600 \mathrm{~m}$ in length and has an inside finished diameter of 3 $\mathrm{m}$. Grouting behind the precast concrete is included and will be accomplished as part of the precast liner installation. This tunnel is intended to be constructed as part of a design/build project with the design costs included in the per meter unit cost. This unit cost was 
developed based on the actual tunnel costs incurred during the construction of the HERA project. The finished tunnel will consist of the interior surface of the precast lining with no provision for lighting, HVAC, electrical distribution, monorail or other interior finishes. (These are included in the 1.1.4 Machine Infrastructure section.)

1.1.3.1.5 Engineering Costs - This element includes the costs for contracted A/E and Construction Management support of 1.1.3.1, Tunnels and Buildings, that is not included in the design/build descriptions indicated above and specifically excludes any additional operational or engineering support from existing DESY personnel.

\subsubsection{Site and Halls and Buildings}

1.1.3.2.1 Site Ground - This element provides for the acquisition of surface land required for the construction of the TESLA project beyond the existing DESY site. Specifically it provides land for the construction of the permanent access shafts and surface buildings at the six cryogenic plant sites distributed along the length of the TESLA tunnel beyond the existing DESY site. Each of these six sites requires approximately $40,000 \mathrm{~m}^{2}$ (10 acres). This element also includes the land needed for the central area, which includes the interaction hall and beam dumps as well as the land required for the XFEL. The central site requires approximately $540,000 \mathrm{~m}^{2}$ (135 acres). The value assigned to this element is based on a prevailing land cost per $\mathrm{m}^{2}$. This per $\mathrm{m}^{2}$ cost includes a nominal provision for connecting to local natural gas, domestic water and sanitary sewer service if available. If these utilities are not locally available, propane, local water wells and sanitary holding tanks or portable toilet facilities will be considered.

1.1.3.2.2 HEP Experimental Hall - This is the single, below ground interaction region hall. The hall will be constructed of cast-in-place concrete and is modeled after the experimental halls provided for the HERA project. The experimental hall measures $82 \mathrm{~m}$ x $32 \mathrm{~m}$ in plan with a ceiling height of $23 \mathrm{~m}$. This provides a total estimated volume of $61,500 \mathrm{~m}^{3}$. The price for this element is based on a unit cost per $\mathrm{m}^{3}$ and is primarily based on the construction experience gained from the HERA project. This hall is intended to be constructed as part of a design/build project with the design costs included in the per $\mathrm{m}^{3}$ unit cost. The finished hall will consist of the interior surface of the cast-in-place concrete with no provision for lighting, HVAC, electrical distribution or other interior finishes.

1.1.3.2.3 Site Infrastructure Auxiliary Halls - This element provides additional local infrastructure support for the seven cryogenic halls and access shafts as well as the single access hall and shaft at the far end of the 
positron Main Linac at Westerhorn. The cost for this element is applied as a lump sum per hall.

1.1.3.2.4 Refrigerator Halls - This element describes the seven surface buildings that enclose the cryogenic equipment and access shafts to the tunnel enclosure below. Each building measures $85 \mathrm{~m}$ x $32 \mathrm{~m}$ in plan with a ceiling height of $10 \mathrm{~m}$. This provides a total estimated volume of 24,360 $\mathrm{m}^{3}$ per building. While general lighting and power distribution is included in this cost, there is no provision for HVAC with respect to comfort cooling or heating in the base cost. It is intended that rejected heat will be utilized to meet winter heating needs. These buildings are intended to be constructed as part of a design/build project with the design costs included in the per $\mathrm{m}^{3}$ unit cost.

1.1.3.2.5 High Energy Dump Halls - These are the two below ground halls that will enclose the electron and positron dumps adjacent to the experimental hall. They will be constructed of cast-in-place concrete and is modeled after the experimental halls provided for the HERA project. Each dump enclosure measures $30 \mathrm{~m}$ x $25 \mathrm{~m}$ in plan with a ceiling height of $15 \mathrm{~m}$. This provides a total estimated volume of $11,250 \mathrm{~m}^{3}$ for each enclosure. The price for this element is based on a unit cost per $\mathrm{m}^{3}$ and is primarily based on the construction experience gained from the HERA project. These enclosures are intended to be constructed as part of a design/build project with the design costs included in the per $\mathrm{m}^{3}$ unit cost. The finished hall will consist of the interior surface of the cast-in-place concrete with no provision for lighting, HVAC, electrical distribution or other interior finishes.

1.1.3.2.6 Cryo Module Test Facility Hall - This is a single surface building that will house the equipment needed to test the completed cryogenic modules prior to final installation. It measures $135 \mathrm{~m} \times 85 \mathrm{~m}$ with a ceiling height of $12 \mathrm{~m}$. This provides a total estimated volume of $137,700 \mathrm{~m}^{3}$. While general lighting and power distribution is included in this cost, there is no provision for HVAC with respect to comfort cooling or heating in the base cost. It is intended that rejected heat will be utilized to meet winter heating needs. These buildings are intended to be constructed as part of a design/build project with the design costs included in the per $\mathrm{m}^{3}$ unit cost.

1.1.3.2.7 RF Modulator and Water Plant Halls - This element describes the even surface buildings that enclose equipment required for the cooling needs of the non-cryogenic portions of the TESLA machine. These buildings will be constructed in conjunction with the Refrigerator Halls described above and measure $35 \mathrm{~m}$ x $35 \mathrm{~m}$ in plan with a ceiling height of $5 \mathrm{~m}$. This provides a total estimated volume of $6,125 \mathrm{~m}^{3}$ per building. While general lighting and power distribution is included in this cost, 
there is no provision for HVAC with respect to comfort cooling or heating in the base cost. It is intended that rejected heat will be utilized to meet winter heating needs. These buildings are intended to be constructed as part of a design/build project with the design costs included in the per $\mathrm{m}^{3}$ unit cost. (Note added, May, 2002: the RF modulator requirements per location are for three floors, each of 1,480

$\mathrm{m}^{2}$ area. This is to be added to the $232 \mathrm{~m}^{2}$ requirement for the water plant as in the TDR.)

1.1.3.2.8 Engineering Cost - This element includes the costs for contracted $\mathrm{A} / \mathrm{E}$ and Construction Management support of 1.1.3.2, Site and Halls and Buildings that are not included in the design/build descriptions indicated above and specifically excludes any additional operational or engineering support from existing DESY personnel.

\subsubsection{General Tunnel Infrastructure}

1.1.3.3.1 Monorail - This monorail system runs the entire length of the machine enclosure. It provides the means for personnel and material transport as well as consideration for emergency use. The cost is based on a unit amount per lineal meter of tunnel enclosure including installation and was developed directly by the monorail manufacturer.

1.1.3.3.2 Traffic Lane - This element provides the traffic lane structure within the Main Linac and damping ring enclosures. It consists of a steel platform built over the distribution cables installed for high voltage and pulse power. It provides for personnel access along the length of the enclosures. The cost is based on a unit amount per lineal meter of tunnel enclosure.

1.1.3.3.3 Fire Safety System - A unit amount per lineal meter of tunnel in included in the estimate to address fire safety issues. It is intended that this system will include a full detection system with only localized suppression and some consideration for remote fire fighting capability with the monorail trains.

1.1.3.3.4 Module Supports - This element provides the support stands for the fully assembled machine modules. They are considered structural supports and therefore part of the conventional construction. The cost is based on a per unit amount that includes both manufacture and installation.

1.1.3.3.5 Electronic Shielding - This element provides for localized radiation shielding of sensitive electronic equipment at various locations along the length of the tunnel. Its cost is based on a unit amount per lineal meter of tunnel enclosure as required. 
1.1.3.3.6 Handy Lossy Cable - This element provides for basic communication needs throughout the underground enclosures. The cost is based on a unit amount per lineal meter of tunnel enclosure.

\subsubsection{Machine Infrastructure}

\subsubsection{Water Plants and Distribution}

1.1.4.3.1 Water in Auxiliary Halls - This element provides the equipment and water source needed at each surface Refrigerator Hall for the cooling water system. It includes required chillers, pumping equipment and piping as well as the drilling of a water well if local domestic water is not available.

1.1.4.3.2 Water in Tunnels - This element provides the equipment needed with the tunnel enclosures for the cooling water system.

1.1.4.3.3 Water at XFEL - This element provided the equipment needed for the XFEL facility requirements for cooling water.

1.1.4.3.4 Water at Experimental Area - This element provides the equipment and water source needed specifically for the cooling water system required at the interaction region. It includes the required chillers, pumping equipment and piping as well as the drilling of a water well if local domestic water is not available.

Elements 1.1.4.3.1 through 1.1.4.3.4 were discussed as a single system and only a total estimated cost for the entire system was indicated.

\subsubsection{AC Power Plant and Distribution}

1.1.4.4.1 Low Voltage AC Power Distribution - This element provides for the installation of all material and equipment needed for power distribution at the $400 \mathrm{~V}$ level for all structures and enclosures both above and below ground.

1.1.4.4.2 High Voltage AC Power Plant - This element provides for the installation of all material and equipment needed for the eight individual connections to local main power distribution supply lines located at each of the cryogenic Refrigerator Halls.

1.1.4.4.3 20 KV AC Power Switchyard Stations - This element provides for the installation of all material and equipment needed for the eight 
individual substations required to transform available local line power to distribution at the $20 \mathrm{KV}$ level.

1.1.4.4.4 Wall Plug Power - This element provides for the installation of all material and equipment needed for wall plug power distribution in all structures and enclosures both above and below ground.

1.1.4.4.5 Low Voltage Power Stations - This element provides for the installation of all material and equipment needed at the eight individual substations required to transform secondary power from the $20 \mathrm{KV}$ level to the $400 \mathrm{~V}$ level.

Elements 1.1.4.4.1 through 1.1.4.4.5 were discussed as a single system and only a total estimated cost for the entire system was indicated.

\subsubsection{Various Supply Systems}

1.1.4.5.1 Main Linac Segment Ventilation - This element provides for the installation of all material and equipment needed to meet the ventilation requirements of the Main Linac enclosures with equipment located at each of the cryogenic Refrigerator Halls.

1.1.4.5.2 Auxiliary Hall Ventilation - This element provides for the installation of all material and equipment needed to meet the ventilation requirements of the surface level cryogenic Refrigerator Halls.

1.1.4.5.3 Main Linac First Station Ventilation - This element provides for the installation of all material and equipment needed to meet the ventilation requirements at the start of each Main Linac enclosure with equipment located at each respective access shaft.

1.1.4.5.4 HEP Experimental Hall Ventilation - This element provides for the installation of all material and equipment needed to meet the ventilation requirements at the interaction region.

1.1.4.5.5 Damping Ring Infrastructure - This element provides for the installation of all material and equipment needed to meet the ventilation requirements at the damping ring enclosures with equipment located at each respective access shaft.

Elements 1.1.4.5.1 through 1.1.4.5.5 were discussed as a single system and only a total estimated cost for the entire system was indicated. 


\section{Comparison of TESLA, CEBAF, and SNS RF Cavities and Cryomodules}

\begin{tabular}{|c|c|c|c|c|}
\hline \multirow[b]{2}{*}{ ITEM } & \multicolumn{2}{|l|}{ SNS } & \multirow[t]{2}{*}{ TESLA } & \multirow[t]{2}{*}{ CEBAF } \\
\hline & Med. B & High B & & \\
\hline Beta & 0.61 & 0.81 & 1 & 1 \\
\hline Frequency, MHz & 805 & 805 & 1300 & 1500 \\
\hline \# Cells per Cavity & 6 & 6 & 9 & 5 \\
\hline Active Length/Cavity, m & 0.68 & 0.91 & 1.04 & 0.5 \\
\hline Cavity Eacc, MV/m & 10.2 & 12.6 & 25 & 5.0 \\
\hline Cavities per Cryomodule & 3 & 4 & 12 & 8 \\
\hline Slot Length, meter & 5.84 & 7.89 & $16 \& 17$ & 10 \\
\hline Cryomodule Field, MV & 21 & 46 & 311 & 20 \\
\hline \# of Cryomodules & 11 & 12 & 1716 & 43 \\
\hline
\end{tabular}

\section{TTF and TESLA RF Cavities and Cryomodules}

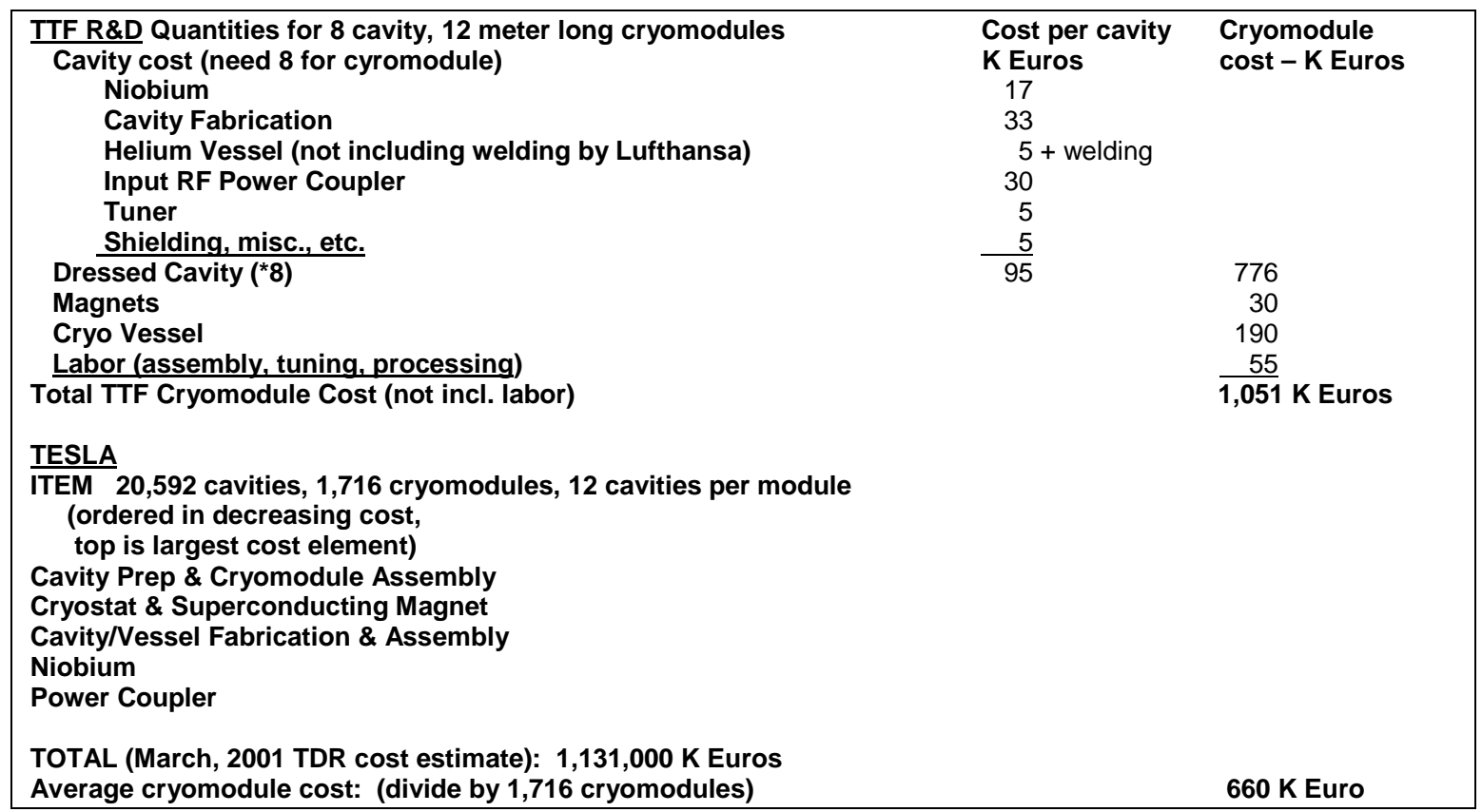




\section{CEBAF RF Cavities and Cryomodules}

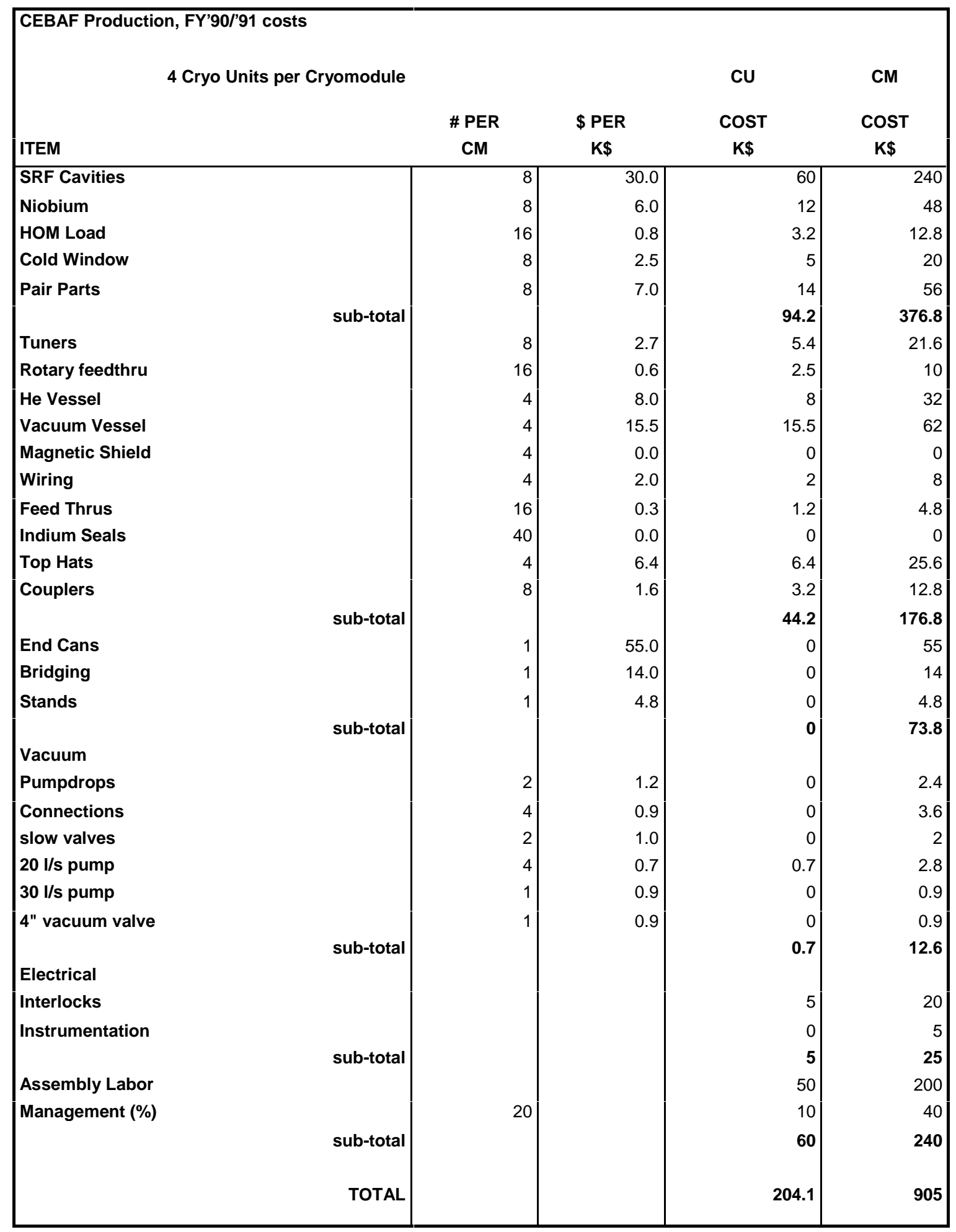

Tesla Engineering Study Review 


\section{SNS RF Cavities and Cryomodules}

\begin{tabular}{|c|c|c|c|c|}
\hline \multicolumn{5}{|l|}{ SNS, FY01/02 costs } \\
\hline \multirow[b]{3}{*}{ ITEM } & \multirow{3}{*}{$\begin{array}{r}\text { Med. B } \\
\text { TOTAL } \\
\text { K\$ }\end{array}$} & \multirow{3}{*}{$\begin{array}{r}\mathrm{CM} \\
\mathrm{COST} \\
\mathrm{K} \$\end{array}$} & \multirow{3}{*}{$\begin{array}{r}\text { High B } \\
\text { TOTAL } \\
\text { K\$ }\end{array}$} & \multirow{3}{*}{$\begin{array}{r}\mathrm{CM} \\
\mathrm{COST} \\
\mathrm{K} \$\end{array}$} \\
\hline & & & & \\
\hline & & & & \\
\hline Cavity Beam Line Assembly & $3,979.9$ & 361.8 & $6,939.5$ & 578.3 \\
\hline Helium Vessel Assembly & 642.6 & 58.4 & $1,080.9$ & 90.1 \\
\hline sub-total & $4,622.4$ & 420.2 & $8,020.4$ & 668.4 \\
\hline Tuner Assembly & 649.4 & 59.0 & 904.2 & 75.3 \\
\hline Header Assemblies & 147.2 & 13.4 & 210.0 & 17.5 \\
\hline Magnetic Shield & 195.6 & 17.8 & 270.8 & 22.6 \\
\hline Thermal Shield Assembly & 392.2 & 35.7 & 530.2 & 44.2 \\
\hline Seals \& Miscellaneous & 56.5 & 5.1 & 70.9 & 5.9 \\
\hline Space Frame & 469.0 & 42.6 & 675.0 & 56.3 \\
\hline Alignment Fiducials & 3.6 & 0.3 & 4.0 & 0.3 \\
\hline Vacuum Tank Assembly & 494.4 & 44.9 & 681.6 & 56.8 \\
\hline sub-total & $2,408.0$ & 218.9 & $3,346.7$ & 278.9 \\
\hline End Can Assembly & $1,470.4$ & 133.7 & $1,557.0$ & 129.8 \\
\hline Stands & 228.0 & 20.7 & 224.9 & 18.7 \\
\hline sub-total & $1,698.4$ & 154.4 & $1,781.9$ & 148.5 \\
\hline Assembly Labor & $1,994.2$ & 181.3 & $3,531.6$ & 294.3 \\
\hline TOTAL & $10,723.1$ & 974.8 & $16,680.7$ & $1,390.1$ \\
\hline
\end{tabular}




\section{TESLA Budget Book - Dictionary Version}

This tabulation is included here, not to provide any additional cost estimate data beyond what is given in the TESLA TDR, but rather to indicate the extent of the elements considered in preparing the cost estimate. This table was put into a WBS spreadsheet format to facilitate viewing the high level summaries while allowing study of the individual elements. The highest level summary is presented, followed by the expanded listing of the elements considered for each sub-system on subsequent pages.

\section{Level 3 Elements}

TESLA Budget Book - Dictionary Version (w/Fermilab WBS)

March 2001 - phg - modified 3dec01

cost estimate in M Euros units - year 2000 costs

includes only level of costs available in TESLA TDR

1 Total - TESLA Collider + XFEL Increments

$\begin{array}{rrr}\text { \# units 1. Cost } & \begin{array}{r}\text { 1.a } \\ \text { cost }\end{array} & \begin{array}{r}1 . a . b \\ \text { cost } \\ \text { roll-up }\end{array} \\ \text { roll-up } & \text { roll-up } & \\ & & \\ 3,377 & & \\ & 3,136 & \\ & & 1,131 \\ & & 587 \\ & & 546 \\ & & 336 \\ & & 124 \\ & & 101 \\ & & 97\end{array}$

1.2 XFEL Increments - total

241

1.2.1 XFEL Increments

241 


\section{Level 4 Elements}

TESLA Budget Book - Dictionary Version (w/Fermilab WBS)

March 2001 - phg - modified 3dec01

cost estimate in M Euros units - year 2000 costs

includes only level of costs available in TESLA TDR

\# units 1.a.b cost

roll-up

1 Total - TESLA Collider + XFEL Increments

1.1 TESLA Collider - total (not including XFEL)

1.1.1 Main Linac Modules

1.1.1.1 Cavity preparation \& string assembly

1.1.1.2 Cryostat modules \& sc magnets

1.1.1.3 Cavity structure \& vessel fabrication

1.1.1.4 Niobium material RRR 300

1.1.1.5 RF Power \& HOM Coupler

1.1.2 Main Linac RF System

1.1.2.1 RF Power Distribution \& LLRF

1.1.2.2 Klystrons \& Interlocks

1.1.2.3 Modulator

1.1.2.4 Pulse transformer \& predriver

1.1.2.5 HV pulse cable \& installation

1.1.3 Tunnel \& Buildings

1.1.3.1 Tunnels \& access shafts

1.1.3.2 Site \& halls \& buildings

1.1.3.3 General Tunnel Infrastructure

1.1.4 Machine Infrastructure

1.1.4.1 Cryogenic plants \& supply

1.1.4.2 Cryogenic Distribution

1.1.4.3 Water plants \& distribution

1.1.4.4 AC Power plant \& distribution

1.1.4.5 Various supply systems

1.1.5 Damping Rings

1.1.5.1 Magnets \& Power System

1.1.5.2 Vacuum system

1.1.5.3 RF Power \& cavity system

1.1.5.4 Beam diagnostics \& controls

1.1.6 Auxiliary Systems

1.1.6.1 Global machine control system

1.1.6.2 Electronic \& cabling

1.1.6.3 Vacuum RF Power coupler

1.1.6.4 Vacuum modules - Main Linac Cryostats

1.1.6.5 Various auxiliary systems

1.1.7 Beam Delivery System

101

Tesla Engineering Study Review 
1.1.7.1 Magnets \& Power System

1.1.7.2 Beam Stops \& cooling

1.1.7.3 Beam diagnostic

1.1.7.4 Vacuum System

1.1.7.5 Fast Kicker \& Collimator System

1.1.8 Injection System

97

1.1.8.1 $5 \mathrm{GeV}$ Positron Linac \& Beamline

1.1.8.2 Positron Source \& Injector

1.1.8.3 $5 \mathrm{GeV}$ Electron Linac

1.1.8.4 Polarized gun \& injector

1.1.8.5 RF Gun \& Injector

1.2 XFEL Increments - total

1.2.1 XFEL Increments

241

1.2.1.1 XFEL Tunnel \& buildings

1.2.1.2 XFEL Machine additives

1.2.1.3 XFEL Beam switchyard

1.2.1.4 Linac to XFEL Beam lines 


\section{Level 5 Elements}

TESLA Budget Book - Dictionary Version (w/Fermilab WBS)

March 2001 - phg - modified 3dec01

cost estimate in M Euros units - year 2000 costs

includes only level of costs available in TESLA TDR

\# units 1.a.b cost

roll-up

1 Total - TESLA Collider + XFEL Increments

1.1 TESLA Collider - total (not including XFEL)

1.1.1 Main Linac Modules

1,131

1.1.1.1 Cavity preparation \& string assembly

1.1.1.1.1 cavity preparation \& assembly

20,592

1.1.1.2 Cryostat modules \& sc magnets

1.1.1.2.1 vacuum vessel \& cold mass $\quad 1,716$

vacuum vessel

cryo supports

GHeRP (return pipe)

shields 4.5 degree

shields 70 degree

cryo pipes

cold mass pre-assembly

intermediate transportation

final transportation

management

1.1.1.2.2 module interconnect beam vacuum $\quad 1,716$

1.1.1.2.3 magnet package $\quad 750$

1.1.1.2.4 module instrumentation $\quad 1,716$

1.1.1.2.5 module interconnection $\quad 1,716$

1.1.1.3 Cavity structure \& vessel fabrication

1.1.1.3.1 Structure machining 20,592

1.1.1.3.2 Tuner mechanics + gear box 20,592

1.1.1.3.3 Titanium vessel, tubes \& bellows 20,592

1.1.1.3.4 Tuner motor \& electronics 20,592

1.1.1.3.5 Assembly \& EB Welding 20,592

1.1.1.3.6 Magnetic shielding Cryoperm 20,592

1.1.1.3.7 Magnetic shielding fabrication 20,592

1.1.1.3.8 Piezo tuner

1.1.1.4 Niobium material RRR 300

1.1.1.4.1 Niobium 300 material 20,592

1.1.1.4.2 Niobium 30 material 20,592

1.1.1.4.3 NbTi flange material 20,592

1.1.1.5 RF Power \& HOM Coupler

1.1.1.5.1 RF power coupler fabrication 20,592

1.1.1.5.2 Cavity control inside 20,592 
1.1.2.1 RF Power Distribution \& LLRF

1.1.2.1.1 RF Power Distribution

RF power circulators

RF power hybrid couplers

$R F$ wave guides

RF transformers

RF bellows

RF signal couplers

1.1.2.1.2 Low Level RF Control

LLC digital feedback

LLC monitoring

LLC RF components

LLC miscellaneous

1.1.2.1.3 (blank)

LLC master oscillator \& distribution

1.1.2.2 Klystrons \& Interlocks

1.1.2.2.1 Klystron, solenoid \& socket 572

1.1.2.2.2 Klystron interlocks 572

1.1.2.2.3 Klys, aux. PS Fil, Sol, Bias, Vac. 572

1.1.2.2.4 (blank)

1.1.2.3 Modulator

1.1.2.3.1 Modulator pulser unit 572

Pulse transformer assembly

HV power supply

Control/interlock

Switch assembly

Crowbar system

Capacitor banks

Bouncer system

Supports, cooling, \& ventilation

Under shoot circuit

Local subsystem control

Miscellaneous

1.1.2.3.2 Modulator interlock

1.1.2.4 Pulse transformer \& predriver

1.1.2.4.1 Pulse trans. Unit (incl .fil. \& container) 572

1.1.2.4.2 Cavity interlock 572

1.1.2.4.3 Tunnel cable connections 572

1.1.2.4.4 PreDriver

1.1.2.5 HV pulse cable \& installation

1.1.2.5.1 Pulse power cables

572

1.1.2.5.2 Pulse cable installation

572 
1.1.3 Tunnel \& Buildings

1.1.3.1 Tunnels \& access shafts

1.1.3.1.1 Main Linac

33,500

1.1.3.1.2 Damping ring

1.1.3.1.3 Access shaft unit

4,000

1.1.3.1.4 Bypass Tunnel positrons

19

1.1.3.1.5 Engineering costs

1.1.3.2 Site \& halls \& buildings

1.1.3.2.1 Site ground

600

1.1.3.2.2 HEP experimental Hall

800,000

1.1.3.2.3 Site infrastructure - aux halls

61,500

1.1.3.2.4 Refrigerator halls

8

1.1.3.2.5 HE dump halls

170,520

1.1.3.2.6 Cryo Module Test Facility Hall

20,000

1.1.3.2.7 RF Modulator \& Water Plant Halls

134,460

1.1.3.2.8 Engineering costs

1.1.3.3 General Tunnel Infrastructure

1.1.3.3.1 Monorail

34,000

1.1.3.3.2 Traffic lane

37,620

1.1.3.3.3 Fire safety system

40,000

1.1.3.3.4 Module supports

3,500

1.1.3.3.5 Electronic shielding

8,237

1.1.3.3.6 Handy lossy cable

40,000

1.1.4 Machine Infrastructure

1.1.4.1 Cryogenic plants \& supply

1.1.4.1.1 Cold boxes

1.1.4.1.2 Compressors

1.1.4.1.3 Distribution boxes 3,4,5,7

1.1.4.1.4 Cold compressors

1.1.4.1.5 Distributions boxes 1,6

1.1.4.1.6 Purification

1.1.4.1.7 Controls

1.1.4.1.8 Liquid Helium storage

1.1.4.1.9 Warm gas storage

1.1.4.1.10 Distribution box 2

1.1.4.1.11 Helium

1.1.4.1.12 Miscellaneous

1.1.4.1.13 Budget value to XFEL Incrementals

1.1.4.2 Cryogenic Distribution

1.1.4.2.1 Warm Helium gas tube (DN200)

1.1.4.2.2 Transfer lines

1.1.4.2.3 Vacuum Barriers

1.1.4.2.4 Feed boxes

1.1.4.2.5 End boxes

1.1.4.2.6 Injection feed boxes

1.1.4.2.7 Damping ring RF feed boxes

1.1.4.2.8 Controls

1.1.4.2.9 Booster

1.1.4.2.10 Injection distribution box

Tesla Engineering Study Review

154

July 8, 2002 (final) version 
1.1.4.3 Water plants \& distribution

1.1.4.3.1 Water auxiliary halls

1.1.4.3.2 Water tunnels

1.1.4.3.3 Water XFEL

1.1.4.3.4 Water Experiments

1.1.4.4 AC Power plant \& distribution

1.1.4.4.1 Low Voltage AC Power distribution

1.1.4.4.2 High Voltage AC Power plant

1.1.4.4.3 $20 \mathrm{KV} \mathrm{AC} \mathrm{power} \mathrm{switchyard} \mathrm{stations}$

1.1.4.4.4 Wall plug power

1.1.4.4.5 Low Voltage AC Power stations

1.1.4.5 Various supply systems

1.1.4.5.1 Main LINC segment ventilation 8

1.1.4.5.2 Auxiliary Hall ventilation 8

1.1.4.5.3 Main Linac first station ventilation 2

1.1.4.5.4 HEP Experimental hall ventilation 1

1.1.4.5.5 Damping Ring Infrastructure

1.1.4.5.6 Cryo Test facility cryogenics

1.1.4.5.7 RF Power test facility

1.1.4.5.8 Cryo test facility operation

1.1.4.5.9 Cryo test RF Power System

1.1.4.5.10 Cryo test control system

1.1.4.5.11 Cryo-connection to HERA cryo-plant

1.1.4.5.12 Cryo test vacuum

1.1.4.5.13 Cryo Test vertical dewar RF 
1.1.5.1 Magnets \& Power System

1.1.5.1.1 Special Magnets - Wigglers

1.1.5.1.2 Quadrupoles for Damping Ring

1.1.5.1.3 Dipoles for Damping Ring

1.1.5.1.4 Injection/Extraction Sections

1.1.5.1.5 Sextupoles for Damping Ring

1.1.5.1.6 Magnet assembly

1.1.5.1.7 Solenoids B-line \& Bunch Compressor

1.1.5.1.8 Magnetic measurements

1.1.5.1.9 Correctors for Damping Ring

1.1.5.1.10 Quadrupoles for Beamline \& B.C.

1.1.5.1.11 multipole girders/supports

1.1.5.1.12 Power supply system

1.1.5.1.13 Dipoles for Beamline and B.C.

1.1.5.1.14 Dipole stands and supports

1.1.5.1.15 Correctors for Beamline \& B.C.

1.1.5.1.16 Sextupoles for Beamline \& B.C.

1.1.5.2 Vacuum system

1.1.5.1.1 Pumps and power supplies

1.1.5.1.2 Damping Ring Vacuum Chamber

1.1.5.1.3 Vacuum chamber supports

1.1.5.1.4 Manual \& auto valves

1.1.5.3 RF Power \& cavity system

1.1.5.3.1 RF Cryo-modules

1.1.5.3.2 RF Power sources

1.1.5.3.3 Waveguide network system

1.1.5.3.4 Cryogenic system

1.1.5.3.5 Cooling system

1.1.5.3.6 Electronics, controls, interlocks, etc.

1.1.5.4 Beam diagnostics \& controls

1.1.5.4.1 BPM button/strip line monitors

1.1.5.4.2 Beam diagnostics electronics

1.1.5.4.3 Toroidal current transformers

1.1.5.4.4 Wall current monitors

1.1.5.4.5 Emittance measurement system

1.1.5.4.6 Tune monitors

1.1.5.4.7 Beam loss monitors

1.1.5.4.8 Fluorescent screens

1.1.5.4.9 Scrapers

1.1.5.4.10 DC current transformers 
1.1.6.1 Global machine control system

1.1.6.1.1 Electronics racks, etc 700

1.1.6.1.2 Crates, front-end servers 700

1.1.6.1.3 Control room equipment 100

1.1.6.1.4 Network equipment 800

1.1.6.1.5 Expendable Materials 300

1.1.6.1.6 Network cables $\quad 500$

1.1.6.1.7 Alarm interface modules 60

1.1.6.1.8 Servers \& consoles $\quad 300$

1.1.6.1.9 Alarm line cables $\quad 60$

1.1.6.2 Electronic \& cabling

1.1.6.2.1 Data acquisition 900

1.1.6.2.2 Monitor electronics Main Linac 800

1.1.6.2.3 Beam loss Main Linac 800

1.1.6.2.4 Beam monitors Main Linac 800

1.1.6.2.5 Monitor electronics general 500

1.1.6.2.6 Commercial electronics 100

1.1.6.2.7 Monitor beam lines 100

1.1.6.3 Vacuum RF Power coupler

1.1.6.3.1 Pump tube $d=100$

1.1.6.3.2 Sputter pump - $60 \mathrm{l} / \mathrm{sec}$

1.1.6.3.3 Titanium sublimation pump

1.1.6.3.4 Sputter pump power supplies

1.1.6.3.5 TSP power supplies

1.1.6.3.6 Manual valve ID 35

1.1.6.3.7 Vacuum component electronics

1.1.6.3.8 Bellows

1.1.6.3.9 Pump pots

1.1.6.4 Vacuum modules - Main Linac Cryostats

1.1.6.4.1 Penning filaments

1.1.6.4.2 Pump stations

1.1.6.4.3 Manual vacuum valve ID 100

1.1.6.4.4 Vacuum installation tools cryostats

1.1.6.4.5 Vacuum component electronics

1.1.6.4.6 Bridge valves

1.1.6.4.7 Pirani filaments

1.1.6.5 Various auxiliary systems

1.1.6.5.1 DC power supplies Main Linac

1.1.6.5.2 Spare Linac modules

1.1.6.5.3 Alignment \& Survey

1.1.6.5.4 Cables from general store

1.1.6.5.5 Connectors from general store

1.1.6.5.6 Main Linac beam vacuum control

1.1.6.5.7 Radiation safety interlock system 
1.1.7 Beam Delivery System

1.1.7.1 Magnets \& Power System

1.1.7.1.1 Magnet Supplies \& Cables

1.1.7.1.2 Quadrupoles 180

1.1.7.1.3 Main Dipoles 370

1.1.7.1.4 Electrostatic Separators 32

1.1.7.1.5 SC Doublets 2

1.1.7.1.6 Sextupoles \& Octupoles 26

1.1.7.1.7 Movers 206

1.1.7.1.8 Emergency extraction quads 22

1.1.7.1.9 Emergency extraction dipoles 12

1.1.7.1.10 Supports 624

1.1.7.1.11 H/V correctors $\quad 400$

1.1.7.1.12 Main extraction quadrupoles $\quad 6$

1.1.7.1.13 Main extraction dipoles 4

1.1.7.1.14 Main extraction septum 2

1.1.7.1.15 Emergency extraction septum 2

1.1.7.2 Beam Stops \& cooling

1.1.7.2.1 Cooling system 2

1.1.7.2.2 Fast extraction, 30 kicker, $1 \mathrm{~m}$ long 2

1.1.7.2.3 Fast sweep 4

1.1.7.2.4 Water vessel 6

1.1.7.3 Beam diagnostic

1.1.7.3.1 Laser profile 2

1.1.7.3.2 Beam electronics $\quad 370$

1.1.7.3.3 Final Focus Monitor 2

1.1.7.3.4 BPM 200

1.1.7.3.5 Beam loss monitor $\quad 370$

1.1.7.3.6 Wire scanner 16

1.1.7.3.7 Cables \& connectors $\quad 370$

1.1.7.4 Vacuum System

1.1.7.4.1 Copper surfacing $\quad 1,800$

1.1.7.4.2 Vacuum tubes 3,400

1.1.7.4.3 Pumps \& valves $\quad 200$

1.1.7.4.4 Electronics 200

1.1.7.4.5 Vacuum utilities 200

1.1.7.5 Fast Kicker \& Collimator System

1.1.7.5.1 Spoiler 26

1.1.7.5.2 Spoiler cooling water unit 26

1.1.7.5.3 Absorber 12

1.1.7.5.4 Absorber cooling water circuit 12

1.1.7.5.5 Instrumentation 2

1.1.7.5.6 General cables \& connectors $\quad 3,400$

1.1.7.5.7 Fast Kickers 16

1.1.7.5.8 Magnet mover electronics \& cables 206

1.1.7.5.9 BPM 10

1.1.7.5.10 Electronics 1

1.1.7.5.11 Miscellaneous 
1.1.8.1 $5 \mathrm{GeV}$ Positron Linac \& Beamline

1.1.8.1.1 Cryogenic modules

1.1.8.1.2 RF power system

1.1.8.1.3 Accelerating system

1.1.8.1.4 Magnet elements

1.1.8.1.5 Beam Line Magnets

1.1.8.1.6 Beam Line Vacuum system

1.1.8.1.7 Beam Line Instrumentation

1.1.8.1.8 Vacuum

1.1.8.1.9 Instrumentation

1.1.8.1.10 Beam Line DC power supplies

1.1.8.2 Positron Source \& Injector

1.1.8.2.1 RF power system

1.1.8.2.2 $100 \mathrm{~m}$ long undulator (all included)

1.1.8.2.3 Accelerating system

1.1.8.2.4 Target \& pulsed magnet (x2)

1.1.8.2.5 Magnets

1.1.8.2.6 Beam Line vacuum

1.1.8.2.7 DC power supplies

1.1.8.2.8 Instrumentation

1.1.8.2.9 Pumps, bellows \& valves

1.1.8.3 $5 \mathrm{GeV}$ Electron Linac

1.1.8.3.1 Cryo Modules

1.1.8.3.2 Klystrons \& Modulators

1.1.8.3.3 Power supplies

1.1.8.3.4 Vacuum

1.1.8.3.5 Magnet packages

1.1.8.4 Polarized gun \& injector

1.1.8.4.1 Room temperature Linac

1.1.8.4.2 SC Linac to $500 \mathrm{MeV}$

1.1.8.4.3 Load-lock system

1.1.8.4.4 Electron gun

1.1.8.4.5 Pre-bunching section

1.1.8.4.6 Matching \& analysis line

1.1.8.5 RF Gun \& Injector

1.1.8.5.1 Equipped cryostat modules

1.1.8.5.2 RF, vacuum \& diagnostics

1.1.8.5.3 $10 \mathrm{MW}$ Klystron \& modulators

1.1.8.5.4 Klystron \& modulator (4.5 MW)

1.1.8.5.5 Laser 
1.2 XFEL Increments - total

1.2.1 XFEL Increments

1.2.1.1 XFEL Tunnel \& buildings

1.2.1.1 XFEL Experimental hall $\quad 150,000$

1.2.1.2 XFEL dump halls $\quad 24,000$

1.2.1.3 XFEL tunnels $\quad 8,000$

1.2.1.4 XFEL tunnel infrastructure $\quad 8,000$

1.2.1.2 XFEL Machine additives

1.2.2.1 Additional air conditioning

1.2.2.2 Radiation safety design \& interlocks

1.2.2.3 XFEL Gun \& Injector

1.2.2.3.1 Equipped cryostat modules

1.2.2.3.2 2x10 MW klystron/modulator assmbl.

1.2.2.3.3 Bunch compressors

1.2.2.3.4 Laser

1.2.2.3.5 3rd harmonic accel. cavity/coupler

1.2.2.3.6 Power supplies

1.2.2.3.7 Preparation chamber (mech\&vac)

1.2.2.3.8 Vacuum \& Diagnostics

1.2.2.4 Additional water plant \& distribution

1.2.2.5 Compressor cavities

120

1.2.2.6 Compressor RF power system

1.2.2.7 Cryogenic distribution additives

1.2.2.8 Wave guide absorber adds

1.2.2.9 Power supply RF modulator adds

1.2.2.10 Substation racks \& electronics

1.2.2.11 Ten Degree bend for XFEL

1.2.2.12 XFEL Bunch compressor 2 \& 3

1.2.2.13 Compressor cryostat module

1.2.1.3 XFEL Beam switchyard

1.2.3.1 XFEL beam dump components

1.2.3.2 Dipole magnets

1.2.3.3 Quadrupole magnets

1.2.3.4 Sextupole Magnets

1.2.3.5 Pulsed dipole magnets

1.2.3.6 Correction magnets

1.2.3.7 Octupole magnets

1.2.3.8 Power supplies \& cables

1.2.3.9 Beam diagnostic \& feedbacks

1.2.3.10 Vacuum System

1.2.3.11 Cable, connectors \& mechanics

1.2.1.4 Linac to XFEL Beam lines

1.2.4.1 Magnets

1.2.4.2 Power supplies \& cables

1.2.4.3 Vacuum per meter

1.2.4.4 Beam position monitors
2,052

57

50

10

295

537

284

6

120

16 